\title{
A New Approach to Transport Coefficients in the Quantum Spin Hall Effect
}

\author{
Giovanna Marcelli, Gianluca Panati® and Stefan Teufel
}

\begin{abstract}
We investigate some foundational issues in the quantum theory of spin transport, in the general case when the unperturbed Hamiltonian operator $H_{0}$ does not commute with the spin operator in view of Rashba interactions, as in the typical models for the quantum spin Hall effect. A gapped periodic one-particle Hamiltonian $H_{0}$ is perturbed by adding a constant electric field of intensity $\varepsilon \ll 1$ in the $j$-th direction, and the linear response in terms of a $S$-current in the $i$-th direction is computed, where $S$ is a generalized spin operator. We derive a general formula for the spin conductivity that covers both the choice of the conventional and of the proper spin current operator. We investigate the independence of the spin conductivity from the choice of the fundamental cell (unit cell consistency), and we isolate a subclass of discrete periodic models where the conventional and the proper $S$-conductivity agree, thus showing that the controversy about the choice of the spin current operator is immaterial as far as models in this class are concerned. As a consequence of the general theory, we obtain that whenever the spin is (almost) conserved, the spin conductivity is (approximately) equal to the spin-Chern number. The method relies on the characterization of a non-equilibrium almoststationary state (NEASS), which well approximates the physical state of the system (in the sense of space-adiabatic perturbation theory) and allows moreover to compute the response of the adiabatic $S$-current as the trace per unit volume of the $S$-current operator times the NEASS. This technique can be applied in a general framework, which includes both discrete and continuum models.
\end{abstract}

\section{Introduction and Main Results}

The aim of this paper is to shed some light on the theory of spin transport in gapped (non-interacting) fermionic systems, a problem which is highly relevant to the research on topological insulators (see the end of Sect. 1.2). 
The theory of spin transport, as compared to charge transport, is still in a preliminary stage. First, despite two decades of scientific debate, no general consensus has been reached yet about the correct form of the operator representing the spin current density. Denoting by $H_{0}$ the unperturbed Hamiltonian operator, by $\mathbf{X}=\left(X_{1}, \ldots, X_{d}\right)$ the position operator and by $S_{z}$ the operator representing the $z$-component of the spin, one may consider ${ }^{1}$

(i) the "conventional" spin current operator

$$
\mathbf{J}_{\text {conv }}^{S_{z}}:=\frac{1}{2}\left(\mathrm{i}\left[H_{0}, \mathbf{X}\right] S_{z}+\mathrm{i} S_{z}\left[H_{0}, \mathbf{X}\right]\right)
$$

which has been used e.g. in $[58,60,64]$;

(ii) the "proper" spin current operator

$$
\mathbf{J}_{\text {prop }}^{S_{z}}:=\mathrm{i}\left[H_{0}, \mathbf{X} S_{z}\right]
$$

proposed in $[62,72]$.

Whenever $\left[H_{0}, S_{z}\right]=0$, the two above definitions agree and the theory of spin transport reduces to the theory of charge transport. However, in general $\left[H_{0}, S_{z}\right] \neq 0$ in topological insulators, as it happens e.g. in the model proposed by Kane and Mele in view of the so-called Rashba term [26,33]. As we will explain now (see the related discussion [43]), the lack of commutativity poses technical and conceptual problems for the theory of spin transport, and the main objective of our paper is to clarify some of these issues.

As a second issue, whenever $\left[H_{0}, S_{z}\right]=0$ the spin conductivity is given, in analogy with charge transport, by a double commutator formula, namely

$$
\sigma_{i j}^{S_{z}}=\mathrm{i} \tau\left(\Pi_{0} S_{z}\left[\left[X_{i}, \Pi_{0}\right],\left[X_{j}, \Pi_{0}\right]\right]\right)
$$

where $\tau$ is the trace per unit volume and $\Pi_{0}$ the Fermi projector of the gapped system. Formula (1.3), equivalently rewritten in terms of Bloch orbitals, has been considered as the starting point for further analysis of the robustness of the spin conductivity $[60,64]$, or for a mathematical comparison of spin conductivity and spin conductance [43]. theory:

In this paper, we address two foundational questions in spin transport

(Q1) is it possible to derive from the first principles of quantum theory, in the general case $\left[H_{0}, S_{z}\right] \neq 0$, a double commutator formula for the spin conductivity similar to (1.3)?

(Q2) to which extent is such a formula affected by a different choice of the spin current operator, namely $\mathbf{J}_{\text {conv }}^{S_{z}}$ versus $\mathbf{J}_{\text {prop }}^{S_{z}}$ ?

Moreover, any formula for spin transport coefficients should satisfy the socalled unit cell consistency (UCC), namely the requirement that any prediction on macroscopic transport must be independent of the choice of the fundamental cell [69].

\footnotetext{
${ }^{1}$ All over the paper we use Hartree units, so that the reduced Planck constant $\hbar$, the mass of the electron $m_{\mathrm{e}}$ and the charge of the positron $e$ are equal to 1 . With this choice, both the unit of charge conductivity $\frac{e^{2}}{h}$ and of spin conductivity $\frac{e}{2 \pi}$ reduce to $\frac{1}{2 \pi}$.
} 
In order to answer these questions, we reconsider the whole approach to quantum transport theory.

\subsection{Two Paradigms for Quantum Transport}

The usual paradigm is based on the adiabatic switching-on of the perturbing electric field. More specifically, one considers the time-dependent Hamiltonian operator

$$
H_{\text {switch }}(t):=H_{0}-f(\eta t) \varepsilon X_{j},
$$

where $f: \mathbb{R} \rightarrow[0,1]$ is a smooth function such that $f(s)=0$ for all $s \leq-1$ and $f(s)=1$ for all $s \geq 0$, i.e. the Hamiltonian describes the process where the perturbation is switched on during the finite time interval $[-1 / \eta, 0]$, for $\eta>0$. As $\eta \rightarrow 0^{+}$, the process becomes adiabatic. One assumes that the system is prepared, at some time $t \leq-1 / \eta$, in the equilibrium state $\Pi_{0}$ and that the switching occurs adiabatically. The state $\rho_{\varepsilon, \eta}(s)$ at macroscopic time $s=\eta t$ is given by the solution to the time-dependent Schrödinger equation

$$
\left\{\begin{array}{l}
\mathrm{i} \eta \frac{\mathrm{d}}{\mathrm{d} s} \rho_{\varepsilon, \eta}(s)=\left[H_{\mathrm{switch}}(s), \rho_{\varepsilon, \eta}(s)\right] \\
\rho_{\varepsilon, \eta}(-1)=\Pi_{0}
\end{array}\right.
$$

The linear response coefficient $\sigma_{A}$ of an extensive observable $A$ is defined by comparing the expectation value of $A$ at time $t_{*} \geq 0$ (when the perturbation is completely switched on) and in the far past (when the system is in the unperturbed equilibrium state). By considering the adiabatic limit, one defines $\sigma_{A}$ by setting

$$
\lim _{\eta \rightarrow 0^{+}} \operatorname{Re} \tau\left(A \rho_{\varepsilon, \eta}\left(t_{*}\right)\right)-\operatorname{Re} \tau\left(A \Pi_{0}\right)=: \varepsilon \sigma_{A}+o(\varepsilon) \quad \text { as } \varepsilon \rightarrow 0 .
$$

The real part appears in the formula since one does not know a priori whether the conditional cyclicity of the trace per unit volume can be invoked. ${ }^{2}$ The standard approach for obtaining a tractable formula for $\sigma_{A}$ is to first approximate $\rho_{\varepsilon, \eta}(0)$ by first-order time-dependent perturbation theory, and then to formally exchange the small field limit and the adiabatic limit, see e.g. $[1,28]$. Choosing $f(\eta t)=\mathrm{e}^{\eta t} \chi_{(-\infty, 0]}(t)+\chi_{(0,+\infty)}(t)$, this results in Kubo's formula [39] for the linear response coefficients ${ }^{3}$

$$
\sigma_{A}^{\text {Kubo }}:=-\mathrm{i} \lim _{\eta \rightarrow 0^{+}} \int_{-\infty}^{0} \mathrm{~d} t \mathrm{e}^{\eta t} \tau\left(\mathrm{e}^{-\mathrm{i} H_{0} t}\left[X_{j}, \Pi_{0}\right] \mathrm{e}^{\mathrm{i} H_{0} t} A\right) .
$$

In the case of charge transport, one considers the response of a charge current in the $i$-th direction, $i \in\{1, \ldots, d\}$, whose corresponding quantum mechanical

\footnotetext{
${ }^{2} \mathrm{~A}$ similar phenomenon appears in quaternionic quantum mechanics, where the Hilbert space trace fails to be cyclic [49].

${ }^{3}$ Note that the specific choice $f(\eta t)=\mathrm{e}^{\eta t}$ for $t \leq 0$ has the computational advantage that the integral in (1.6) becomes the inverse Liouvillian, i.e. that the right hand side of (1.6) for finite $\eta>0$ equals, at least formally, $\tau\left(\left(\mathcal{L}_{H_{0}}-\mathrm{i} \eta\right)^{-1}\left(\left[X_{j}, \Pi_{0}\right]\right) A\right)$. However, in the adiabatic limit any other integrable and smooth choice for the switching function $f$ leads to the same value for $\sigma_{A}^{\text {Kubo. }}$.
} 
operator is $J_{i}^{\mathrm{c}}:=\mathrm{i}\left[H_{0}, X_{i}\right]$, and from (1.6) one obtains the formula

$$
\sigma_{J_{i}^{\mathrm{c}}}^{\mathrm{Kubo}}=\mathrm{i} \tau\left(\Pi_{0}\left[\left[X_{i}, \Pi_{0}\right],\left[X_{j}, \Pi_{0}\right]\right]\right) .
$$

The importance of the double commutator formula (1.7) (sometimes dubbed Kubo-Chern formula) cannot be overstated, as it implies e.g. quantization of Hall conductivity in two-dimensional systems [5,10,22,37]. When considering spin transport, we had to face the fact that even the algebra which leads formally to (1.7) becomes cumbersome for spin currents, whenever $\left[H_{0}, S_{z}\right] \neq$ 0 . Moreover, the fact that the formula is intrinsic (i.e. does not depend on the choice of the switching function appearing in (1.4) and on the choice of $t_{*} \geq 0$ ) is not obvious as far as spin currents are concerned.

Thus, we propose an alternative way of computing linear response coefficients based on the non-equilibrium almost-stationary states (NEASS) (see Sect. 4), a concept related to the almost-invariant subspaces in space-adiabatic perturbation theory $[52,53,67]$. Assuming, for the moment, that the state of the system at times when the perturbation has been turned on is approximately given by the NEASS $\Pi^{\varepsilon}$, we find a simple prescription for computing linear (and also higher order) response coefficients: Let $\Pi^{\varepsilon}=\Pi_{0}+\varepsilon \Pi_{1}+o(\varepsilon)$, then from

$$
\tau\left(A \Pi^{\varepsilon}\right)-\tau\left(A \Pi_{0}\right)=\varepsilon \tau\left(A \Pi_{1}\right)+o(\varepsilon)
$$

one concludes that

$$
\sigma_{A}=\operatorname{Re} \tau\left(A \Pi_{1}\right) .
$$

In this paper, we will show how to compute formulas for the spin-conductivities based on formula (1.8) for linear response coefficients, instead of (1.6). The advantage of this method is that the operator $\Pi_{1}$ is rather explicit, namely $\Pi_{1}=\mathcal{I}\left(\overline{\left[X_{j}, \Pi_{0}\right]}\right)$, where the overline denotes the operator closure and $\mathcal{I}$ is the inverse of the Liouvillian operator $B \mapsto\left[H_{0}, B\right]$, with integral representation (4.2).

Of course one expects, and formally it is also easy to see, that the two expressions (1.6) and (1.8) agree. However, in the present setting-where expectations are obtained via a trace per unit volume which is only conditionally cyclic - this is not straightforward to prove. Moreover, both formulas are somewhat heuristic: For (1.6) we assumed applicability of time-dependent perturbation theory also for long adiabatic time-scales, while for (1.8) we just postulated that the perturbed system is in the state $\Pi^{\varepsilon}$.

In order to reconcile and justify both approaches, one needs to prove that in the adiabatic regime the dynamical switching drives the initial equilibrium state $\Pi_{0}$ approximately into the NEASS $\Pi^{\varepsilon}$, i.e. that the state $\rho_{\varepsilon, \eta}(t)$ is close to $\Pi^{\varepsilon}$. Indeed, it is shown in [41] that for times $t \geq 0$ and any $n, m \in \mathbb{N}^{*}$

$$
\left.\sup _{\eta \in I_{m, \varepsilon}}\left|\tau\left(A \rho_{\varepsilon, \eta}(t)\right)-\tau\left(A \Pi^{\varepsilon}\right)\right|=\mathcal{O}\left(\varepsilon^{n}\right) \quad \text { (for } t \geq 0\right)
$$

uniformly on bounded intervals in (macroscopic) time. Here $I_{m, \varepsilon}=\left[\varepsilon^{m}, \varepsilon^{1 / m}\right]$ is an interval of admissible time-scales for the switching. Too slow switching $\left(\eta \ll \varepsilon^{m}\right.$ for all $\left.m \in \mathbb{N}^{*}\right)$ must be excluded, because due to tunneling the 
NEASS decays on such long times-scales, while too fast switching $(1 \gg \eta \gg$ $\varepsilon^{1 / m}$ for all $m \in \mathbb{N}^{*}$ ) would merely yield an error $o(1)$ on the right hand side of (1.9) (see also $[28,68]$ ). In other words, the initial equilibrium state $\Pi_{0}$ dynamically evolves into the NEASS independently of the shape of the switching function up to lower order errors.

\subsection{Main Results on Spin Transport and Conductivity}

By using the NEASS paradigm, we will answer the questions $\left(Q_{1}\right)$ and $\left(Q_{2}\right)$ stated before, at least in the periodic setting. Let us shortly summarize the main results in the paper.

We consider a crystalline system of non-interacting fermions, whose onebody Hamiltonian $H_{0}$ is periodic. This operator acts on the Hilbert space $\mathcal{H}=L^{2}(\mathcal{X}) \otimes \mathbb{C}^{N}$, where either $\mathcal{X}=\mathbb{R}^{d}$ (continuum case) or $\mathcal{X} \subset \mathbb{R}^{d}$ is a discrete set (discrete case), and $N$ is the number of internal degrees of freedom of the particle, which may include spin; periodicity of $H_{0}$ is understood with respect to (magnetic) translations along vectors in a Bravais lattice $\Gamma \simeq \mathbb{Z}^{d}$. We assume that the Hamiltonian $H_{0}$ has a spectral gap and that the initial state of the system is given by the spectral projection $\Pi_{0}$ on the bands below this gap (Fermi projector). The system is driven out of equilibrium by applying a constant electric field of intensity $\varepsilon \ll 1$ pointing in the $j$-th direction, $j \in\{1, \ldots, d\}$. Hence, the stationary Hamiltonian of the perturbed system is $H^{\varepsilon}=H_{0}-\varepsilon X_{j}$, where $X_{j}$ is the $j$-th component of the position operator.

We consider a generalized spin operator in the form $S=\mathbb{1}_{L^{2}(\mathcal{X})} \otimes s$ anddenoting by $J_{\text {conv }, i}^{S}:=\frac{1}{2}\left(\mathrm{i}\left[H_{0}, X_{i}\right] S+\mathrm{i} S\left[H_{0}, X_{i}\right]\right)$ and $J_{\text {prop }, i}^{S}=\mathrm{i}\left[H_{0}, X_{i} S\right]$ the corresponding conventional and proper $S$-current operator - we define the conventional and proper $S$-conductivity, respectively, $\mathrm{as}^{4}$

$$
\operatorname{Re} \tau\left(J_{\text {conv } / \text { prop }, i}^{S} \Pi^{\varepsilon}\right)-\operatorname{Re} \tau\left(J_{\text {conv } / \text { prop }, i}^{S} \Pi_{0}\right)=: \varepsilon \sigma_{\text {conv } / \text { prop }, i j}^{S}+o(\varepsilon) .
$$

In view of the controversy on the choice of the spin current operator discussed at the beginning of Introduction, we find convenient the decomposition

$$
J_{\text {prop }, i}^{S} \Pi_{1}=\mathrm{i}\left[H_{0}, X_{i} S\right] \Pi_{1}=\mathrm{i}\left[H_{0}, X_{i}\right] S \Pi_{1}+X_{i}\left(\mathrm{i}\left[H_{0}, S\right]\right) \Pi_{1}=\mathrm{O}+X_{i} \mathrm{R}
$$

where we have defined the operators

$$
\mathrm{O}:=\mathrm{i}\left[H_{0}, X_{i}\right] S \Pi_{1} \quad \text { and } \quad \mathrm{R}:=\mathrm{i}\left[H_{0}, S\right] \Pi_{1} .
$$

In this decomposition, the $S$-orbital term O contains the contribution associated with the conventional $S$-current operator, while the $S$-rotation term $X_{i} \mathrm{R}$ contains corrections related to the replacement of the latter with the proper $S$-current operator. More precisely, we prove in Theorem 5.6 that splitting (1.11) leads to

$$
\sigma_{\text {prop }, i j}^{S}=\sigma_{\text {conv }, i j}^{S}+\sigma_{\text {rot }, i j}^{S}
$$

\footnotetext{
${ }^{4}$ Notice that the $j$-dependence of $\sigma_{\text {conv } / \text { prop }, i j}^{S}$ is hidden on the left-hand side of the following definition in $\Pi_{1}$, see its definition in Proposition 4.1.2.
} 
where

$$
\begin{aligned}
\sigma_{\text {conv }, i j}^{S}= & \operatorname{Re} \tau\left(\mathrm{i} \Pi_{0}\left[\left[X_{i}, \Pi_{0}\right] S,\left[X_{j}, \Pi_{0}\right]\right]\right) \\
& +\operatorname{Re} \tau\left(\mathrm{i}\left[H_{0}, X_{i}^{\mathrm{D}}\right] S^{\mathrm{OD}} \Pi_{1}+\mathrm{i} X_{i}^{\mathrm{OD}}\left[S, H_{0}\right] \Pi_{1}\right),
\end{aligned}
$$

with $A^{\mathrm{D}}$ (resp. $A^{\mathrm{OD}}$ ) referring to the diagonal (resp. off-diagonal) part of the operator $A$ with respect to the orthogonal decomposition induced by $\Pi_{0}$, and the rotation $S$-conductivity is

$$
\sigma_{\text {rot }, i j}^{S}=\operatorname{Re} \tau\left(X_{i} \mathrm{R}\right)=\operatorname{Re} \tau\left(\mathrm{i} X_{i}\left[H_{0}, S\right] \Pi_{1}\right) .
$$

Notice that the first line of (1.14) is in the form of a current-current correlation at the equilibrium, involving the conventional $S$-current and the charge current, while the second line involves $\Pi_{1}$. Moreover, the trace per unit volume in (1.14) and (1.15) can be replaced with the ordinary trace of the operator restricted to the fundamental cell, up to a volume factor, as in the statement of Theorem 5.6, even if the operator appearing in (1.15) is not periodic.

In order to analyze the $S$-rotation contribution $\sigma_{\text {rot, } i j}^{S}$, we preliminary prove in Proposition 5.4.1 that for any bounded periodic observable $B$, satisfying suitable regularity properties, the expectation of the B-torque operator $\mathrm{i}\left[H_{0}, B\right]$ on $\Pi_{1}$ is given by a double commutator formula, namely

$$
\tau\left(\mathrm{i}\left[H_{0}, B\right] \Pi_{1}\right)=\tau(\underbrace{\mathrm{i} \Pi_{0}\left[\left[\Pi_{0}, B\right],\left[\Pi_{0}, X_{j}\right]\right]}_{\mathcal{T}_{B}}),
$$

where the operator $\mathcal{T}_{B}$ may be dubbed $B$-torque response in agreement with [43]. If, in addition, $\left[B, X_{j}\right]=0$ then $\tau\left(\mathrm{i}\left[H_{0}, B\right] \Pi_{1}\right)=0$, as stated in Proposition 5.4.2. Physically, this result means that even if $\mathrm{i}\left[H_{0}, B\right] \neq 0$, the fact that $B$ commutes with the perturbation $-\varepsilon X_{j}$ implies the mesoscopic conservation of the observable $B$, at least within first order approximation in the NEASS. In particular, when $B=S_{z}$ (or for any generalized spin operator $S$, see Corollary 5.5), we have that the expectation of the spin-torque on $\Pi_{1}$ equals the expectation of the spin-torque response $\mathcal{T}_{S_{z}}$ and that the latter vanishes, in agreement with [43, Theorem 2.8]. Notice that the vanishing of the expectation of the spin-torque response is a condition singled out in [43] to obtain the equality of spin conductivity and spin conductance in two-dimensional systems.

As a further step, we consider the unit cell consistency (UCC) of both the contributions to the proper $S$-conductivity appearing in (1.13). We prove in Proposition 5.8 that $\sigma_{\text {conv }}^{S}$ always satisfies UCC, while for the additional contribution $\sigma_{\text {rot }}^{S}$ we can prove UCC only if the model enjoys a discrete rotational symmetry, in agreement with the claim in [62] that the use of $\mathbf{J}_{\text {prop }}^{S_{z}}$ is "possible for systems where the spin generation in the bulk is absent due to symmetry reasons". In Proposition 5.9, we isolate a subclass of discrete models, enjoying a discrete rotational symmetry and a further property, such that $\sigma_{\text {conv }}^{S}=\sigma_{\text {prop }}^{S}$. Remarkably, the paradigmatic model proposed by Kane and Mele is in this class. A crucial consequence is that, for this class of models, the 
choice of the spin current operator (either $\mathbf{J}_{\text {conv }}^{S}$ or $\mathbf{J}_{\text {prop }}^{S}$ ) is immaterial as far as the $S$-conductivity is concerned.

While the paper is focused on transport theory, one of our long-term goals is to clarify the relation between the spin transport coefficients and the topological invariants associated with quantum spin Hall (QSH) insulators. These materials, theoretically predicted in $[33,34]$ and soon experimentally realized $[38,65]$, display dissipationless edge spin currents, which are robust against continuous deformations of the model and disorder [60]. A crucial issue, both for fundamental understanding and for potential applications, is whether there exists a bulk topological invariant "protecting" the QSH effect. Two candidates have been extensively investigated in the literature. First, the $\mathbb{Z}_{2}$-valued index proposed by Fu, Kane and Mele [21,34], whose definition and geometric properties rely on the fermionic time-reversal symmetry of the system $[15,20,23]$. Second, the (half-)integer-valued spin-Chern number, introduced in [61] via spin dependent boundary conditions, and later intrinsically redefined by Prodan as a bulk invariant [56], which relies instead on the almost-conservation of spin, and is associated with robust spin edge currents $[36,55,58,59]$.

Our analysis establishes a direct relation between the bulk spin conductivity and the spin-Chern number, in agreement with the (recent) discovery that QSH plateaux may persist under broken time-reversal symmetry [18]. Indeed, whenever spin is conserved, our results yield that the (bulk) spin conductivity equals the spin-Chern number (Remark 5.12). Moreover, the result is robust: If spin is approximately conserved, with errors of order $\mathcal{O}(\lambda)$, then the mentioned equality holds true up to a correction of order $\mathcal{O}(\lambda)$ (Proposition 5.13), in analogy with the persistence of edge spin currents proved in [58].

In summary, our paper contributes to put spin transport theory on a firm mathematical ground: We derive a new formula for the spin conductivity which covers both the choice of the conventional and the proper spin current operator; we isolate conditions under which UCC is satisfied and additional conditions which guarantee that $\sigma_{\text {conv }}^{S}=\sigma_{\text {prop }}^{S}$; we make connection with the spin-Chern number. We hope that our mathematical investigations will contribute to clarify some of the controversies in the emerging and promising field of spintronics, and will stimulate a fruitful exchange of ideas between mathematicians and solid state physicists. While, for technical reasons, this paper focuses on the case of periodic non-interacting systems, we are confident that our approach can be suitably generalized to random and interacting systems.

\subsection{Further References to the Literature}

Several different mathematical problems have been labeled "proving Kubo's formula" and a short review highlighting the differences appears elsewhere [28]. Here we only mention a few works without going into any detail: A similar approach to the one we use was employed in [66], where Kubo's formula for the Hall conductivity of simple isolated bands is derived using semi-classical methods. The rigorous derivation of Kubo's formula for interacting fermionic systems on the lattice has recently been done in $[7,46,68]$, where $[7,46]$ consider only situations where the perturbation does not close the spectral gap. A 
similar result for non-interacting fermions in the continuum is in preparation $[42,43]$, generalizing a previous result [19] which also assumes a non-closinggap condition. In many other works, Kubo's formula for the Hall conductivity is taken as a starting point and the objective is to prove quantization of the Hall plateaux also in presence of disorder, assuming the Fermi energy lies in a mobility gap $[1,10,12]$, or including interaction effects $[6,24,27]$, with the aim of proving universality of the Hall conductivity. Moreover, the linear response to a quenched perturbation has been recently analyzed in [14]. Finally, in [13, 17] (and references therein) mathematical frameworks are developed, within which the applicability of linear response theory in very general random resp. interacting systems can be established. However, a rigorous justification of Kubo's formula for the quantum Hall conductivity in situations with mobility gap is still a completely open problem, even in the case of non-interacting systems on the lattice.

Linear response theory can also be considered in the case of heat or charge fluxes induced by thermodynamical (i.e. non-mechanical) driving forces, such as deviations of temperature or chemical potential from their equilibrium values. In this context, the validity of the Green-Kubo formula has been extensively investigated in algebraic quantum statistical mechanics, by relating it to the structure of non-equilibrium steady states [29-32].

The field of spintronics and of quantum transport of spin is relatively new, but has already attracted a lot of attention both in the physics and mathematics communities. Results concerning the quantization and robustness of spin Hall currents in the presence of disorder $[56,58]$ and of interactions $[3$, 44] also rely, to some extent, on a Kubo-like formula. We foresee the possibility of adapting the techniques developed in $[46,68]$ to derive such formulas from first principles also in the context of interacting fermions on a lattice.

\section{Periodic Operators and Trace Per Unit Volume}

In condensed matter physics, it is customary to describe crystalline solids by means of periodic Hamiltonian operators. The appropriate trace-like functional used to compute thermodynamic expectations of periodic observables is given by the trace per unit volume. This Section is devoted to recall some generalities about this framework.

Let $\mathcal{X}$ denote the configuration space of a $d$-dimensional crystal. We will treat both continuum models, in which $\mathcal{X}=\mathbb{R}^{d}$ equipped with the Lebesgue measure, and discrete models, in which $\mathcal{X} \subset \mathbb{R}^{d}$ is a discrete set of points arranged in a crystalline structure, equipped with the counting measure (in $d=$ 2 think of the square lattice $\mathbb{Z}^{2}$ or of the honeycomb structure, for example). In general, "crystalline structure" means that we assume the existence of a Bravais lattice

$$
\Gamma=\operatorname{Span}_{\mathbb{Z}}\left\{a_{1}, \ldots, a_{d}\right\} \simeq \mathbb{Z}^{d}
$$

that acts on $\mathcal{X}$ by translations, i.e. $\mathrm{T}_{\gamma} x:=x+\gamma$ for $\gamma \in \Gamma$ defines a group action $\mathrm{T}: \Gamma \times \mathcal{X} \rightarrow \mathcal{X}$. 
We consider the one-particle Hilbert space

$$
\mathcal{H}=L^{2}(\mathcal{X}) \otimes \mathbb{C}^{N} \simeq L^{2}\left(\mathcal{X}, \mathbb{C}^{N}\right)
$$

for a particle moving on $\mathcal{X}$ and having $N$ internal degrees of freedom (e.g. spin). In the following we will write elements of $\mathcal{H}$ as $\mathbb{C}^{N}$-valued functions on $\mathcal{X}$. We assume that there is a unitary representation $T$ of $\Gamma$ on $\mathcal{H}$ by (magnetic) translation operators

$$
\left(T_{\gamma} \psi\right)(x):=M(\gamma, x) \psi(x-\gamma), \quad \text { for all } \gamma \in \Gamma \text { and } \psi \in \mathcal{H},
$$

where $M: \Gamma \times \mathcal{X} \rightarrow \mathcal{U}\left(\mathbb{C}^{N}\right)$ are unitaries satisfying the cocycle condition ${ }^{5}$

$$
M\left(\gamma_{1}+\gamma_{2}, x\right)=M\left(\gamma_{2}, x\right) M\left(\gamma_{1}, x-\gamma_{2}\right) \text { for all } \gamma_{1}, \gamma_{2} \in \Gamma \text { and } x \in \mathcal{X} .
$$

Position operators for $j \in\{1, \ldots, d\}$ are defined via

$$
\left(X_{j} \psi\right)(x):=x_{j} \psi(x), \quad \text { for all } \psi \in \mathcal{D}\left(X_{j}\right) .
$$

An operator $A$ on $\mathcal{H}$ is called periodic or, more specifically, $\Gamma$-periodic if $\left[A, T_{\gamma}\right]=0$ for all $\gamma \in \Gamma$. The following simple observation, whose proof is omitted, is very useful.

Lemma 2.1. For any periodic operator $A$, the operator $\left[A, X_{j}\right]$ is also periodic.

Notice that, in general, the operator $\left[A, X_{j}\right]$ might be non-densely defined or even defined on the trivial subspace $\{0\}$, as pointed out in [35, III- $\S 5.1]$. This pathology will not appear for the specific operators we will consider in the following sections.

The analysis of periodic operators is best performed in the so-called (magnetic) Bloch-Floquet-Zak representation (see e.g. $[40,45,50]$ and references therein). The (magnetic) Bloch-Floquet-Zak transform is initially defined on compactly supported functions $\psi \in C_{0}\left(\mathcal{X}, \mathbb{C}^{N}\right) \subset L^{2}\left(\mathcal{X}, \mathbb{C}^{N}\right)$ as

$$
\left(\mathcal{U}_{\mathrm{BF}} \psi\right)(k, y):=\mathrm{e}^{-\mathrm{i} k \cdot y} \sum_{\gamma \in \Gamma} \mathrm{e}^{\mathrm{i} k \cdot \gamma}\left(T_{\gamma} \psi\right)(y) \quad k \in \mathbb{R}^{d}, y \in \mathcal{X} .
$$

By construction, for fixed $k \in \mathbb{R}^{d}$, the function $\left(\mathcal{U}_{\mathrm{BF}} \psi\right)(k, \cdot)$ is periodic with respect to the magnetic translations (2.2); hence, it defines an element in the Hilbert space

$$
\begin{aligned}
\mathcal{H}_{\mathrm{f}} & :=\left\{\varphi \in L_{\mathrm{loc}}^{2}\left(\mathcal{X}, \mathbb{C}^{N}\right) \mid T_{\gamma} \varphi=\varphi \text { for all } \gamma \in \Gamma\right\} \quad \text { with } \\
\|\varphi\|_{\mathcal{H}_{\mathrm{f}}}^{2} & :=\int_{\mathcal{C}_{1}} \mathrm{~d} y|\varphi(y)|^{2},
\end{aligned}
$$

where the norm refers to a fundamental cell $\mathcal{C}_{1}$ for $\Gamma$ (see $(2.7)$ ). As functions of $k$, elements in the range of $\mathcal{U}_{\mathrm{BF}}$ are not periodic with respect to the reciprocal lattice $\Gamma^{*}$, but rather $\varrho$-equivariant, namely

$$
\left(\mathcal{U}_{\mathrm{BF}} \psi\right)\left(k+\gamma^{*}, y\right)=\varrho\left(\gamma^{*}\right)\left(\mathcal{U}_{\mathrm{BF}} \psi\right)(k, y) \text { for all } \gamma^{*} \in \Gamma^{*},
$$

\footnotetext{
${ }^{5}$ The case of magnetic translations [71] is included in this framework, and thus, the BlochLandau Hamiltonian can be considered in our setting, assuming a rationality condition on the magnetic flux per unit cell.
} 
where $^{6}$

$$
\varrho: \Gamma^{*} \rightarrow \mathcal{U}\left(\mathcal{H}_{\mathrm{f}}\right), \quad\left(\varrho\left(\gamma^{*}\right) \varphi\right)(y):=\mathrm{e}^{-\mathrm{i} \gamma^{*} \cdot y} \varphi(y),
$$

defines a unitary representation of $\Gamma^{*}$ on $\mathcal{H}_{\mathrm{f}}$. The map defined by (2.4) extends to a unitary operator

$$
\mathcal{U}_{\mathrm{BF}}: \mathcal{H} \rightarrow \mathcal{H}_{\varrho},
$$

where $\mathcal{H}_{\varrho} \equiv L_{\varrho}^{2}\left(\mathbb{R}^{d}, \mathcal{H}_{\mathrm{f}}\right)$ is the space of locally- $L^{2}, \mathcal{H}_{\mathrm{f}}$-valued, $\varrho$-equivariant functions on $\mathbb{R}^{d}$. Denoting by $\mathbb{B}^{d}$ a fundamental domain for $\Gamma^{*}$, the inverse transformation $\mathcal{U}_{\mathrm{BF}}^{-1}: \mathcal{H}_{\varrho} \rightarrow \mathcal{H}$, sometimes dubbed Wannier transform, is explicitly given by

$$
\left(\mathcal{U}_{\mathrm{BF}}^{-1} \varphi\right)(x)=\frac{1}{\left|\mathbb{B}^{d}\right|} \int_{\mathbb{B}^{d}} \mathrm{~d} k \mathrm{e}^{\mathrm{i} k \cdot x} \varphi(k, x) .
$$

At least formally, a periodic operator $A$ on $\mathcal{H}$ becomes a covariant fibered operator on $\mathcal{H}_{\varrho}$. More precisely, taking into account the following inclusion and natural isomorphism

$$
L_{\varrho}^{2}\left(\mathbb{R}^{d}, \mathcal{H}_{\mathrm{f}}\right) \subset L^{2}\left(\mathbb{R}^{d}, \mathcal{H}_{\mathrm{f}}\right) \simeq \int_{\mathbb{R}^{d}}^{\oplus} \mathrm{d} k \mathcal{H}_{\mathrm{f}}
$$

one has

$$
\mathcal{U}_{\mathrm{BF}} A \mathcal{U}_{\mathrm{BF}}^{-1}=\int_{\mathbb{R}^{d}}^{\oplus} \mathrm{d} k A(k),
$$

where each $A(k)$ acts on $\mathcal{H}_{\mathrm{f}}$ and satisfies the covariance property $A\left(k+\gamma^{*}\right)=$ $\varrho\left(\gamma^{*}\right) A(k) \varrho\left(\gamma^{*}\right)^{-1}$ for all $k \in \mathbb{R}^{d}$ and $\gamma^{*} \in \Gamma^{*}$.

Most relevant extensive observables in crystalline systems are periodic self-adjoint operators. However, in an infinite system neither these periodic extensive observables nor translation invariant states are trace class. The appropriate functional is instead given by the trace per unit volume $\tau$, which is well suited to take into account invariance or covariance by discrete lattice translations in the setting of periodic or more generally ergodic operators $[2,9,12,54]$. The trace per unit volume is defined as follows (compare [12, Prop. 3.20]). Denote by $\chi_{\Omega}$ the orthogonal projection on $\mathcal{H}$ which multiplies by the characteristic function of $\Omega \subset \mathcal{X}$. For any $L \in 2 \mathbb{N}+1$, we set

$$
\mathcal{C}_{L}:=\left\{x \in \mathcal{X}: x=\sum_{j=1}^{d} \alpha_{j} a_{j} \text { with }\left|\alpha_{j}\right| \leq L / 2 \forall j \in\{1, \ldots, d\}\right\}
$$

and $\chi_{L}:=\chi_{\mathcal{C}_{L}}$. The set $\mathcal{C}_{1}$ is called a fundamental or primitive (unit) cell. It is not unique since the choice of the spanning vectors $\left\{a_{j}\right\}_{1 \leq j \leq d}$ for $\Gamma$ (see (2.1)) is not unique. Notice that, restricting to odd integers $L \in 2 \mathbb{N}+1$, one has the convenient decomposition ${ }^{7}$

$$
\mathcal{C}_{L}=\bigsqcup_{\gamma \in \Gamma \cap \mathcal{C}_{L}} \mathrm{~T}_{\gamma} \mathcal{C}_{1}
$$

\footnotetext{
${ }^{6}$ We denote by $\mathcal{U}\left(\mathcal{H}_{\mathrm{f}}\right)$ the group of the unitary operators on $\mathcal{H}_{\mathrm{f}}$.

${ }^{7}$ The symbol $\bigsqcup$ denotes the disjoint union up to zero-measure sets.
} 
We call an operator $A$ acting in $\mathcal{H}$ trace class on compact sets if $\chi_{K} A_{\chi_{K}}$ is trace class for all compact sets $K \subset \mathcal{X}{ }^{8}$

Definition 2.2 (Trace per unit volume). Let $A$ be an operator acting in $\mathcal{H}$ such that $A$ is trace class on compact sets. The trace per unit volume of $A$ is defined as

$$
\tau(A):=\lim _{\substack{L \rightarrow \infty \\ L \in 2 \mathbb{N}+1}} \frac{1}{\left|\mathcal{C}_{L}\right|} \operatorname{Tr}\left(\chi_{L} A \chi_{L}\right),
$$

whenever the limit exists.

Let us denote

$$
\begin{aligned}
& \mathcal{B}_{\infty}^{\tau}:=\{\text { bounded periodic operators on } \mathcal{H}\} \\
& \mathcal{B}_{1}^{\tau}:=\left\{A \in \mathcal{B}_{\infty}^{\tau} \text { such that }\|A\|_{1, \tau}:=\tau(|A|)<\infty\right\} .
\end{aligned}
$$

We will refer to operators in $\mathcal{B}_{1}^{\tau}$ as the operators of trace-per-unit-volume class or $\tau$-class for simplicity. Moreover, in view of [12, Proposition 3.17] we have $\mathcal{B}_{\infty}^{\tau} \cdot \mathcal{B}_{1}^{\tau} \subset \mathcal{B}_{1}^{\tau}$ and $\mathcal{B}_{1}^{\tau} \cdot \mathcal{B}_{\infty}^{\tau} \subset \mathcal{B}_{1}^{\tau}$, and

$$
\|A B\|_{1, \tau} \leq\|A\|_{1, \tau}\|B\| \text { and }\|B A\|_{1, \tau} \leq\|A\|_{1, \tau}\|B\| \quad \forall A \in \mathcal{B}_{1}^{\tau}, B \in \mathcal{B}_{\infty}^{\tau} .
$$

The following lemma recalls some useful properties of $\tau$-class operators.

Lemma 2.3. Let $A \in \mathcal{B}_{1}^{\tau}$. Then

$$
\operatorname{Tr}\left(\left|\chi_{1} A \chi_{1}\right|\right) \leq\|A\|_{1, \tau}
$$

and

$$
\operatorname{Tr}\left(\left|\chi_{L} A \chi_{L}\right|\right)<\infty \quad \forall L \in 2 \mathbb{N}+1 .
$$

In particular, we have that $A$ is trace class on compact sets.

Proof. Inequality (2.11) is proved in [12, Lemma 3.10] and the proof given there easily generalizes to obtain also (2.12).

The next result allows to compute the trace per unit volume of operators which are periodic and trace class on compact sets.

Proposition 2.4. 1. Let $A$ be periodic and trace class on compact sets ${ }^{9}$. Then, $\tau(A)$ is well defined and

$$
\tau(A)=\frac{1}{\left|\mathcal{C}_{1}\right|} \operatorname{Tr}\left(\chi_{1} A \chi_{1}\right)
$$

\footnotetext{
${ }^{8}$ This condition is automatically satisfied in the discrete case for any operator $A$, since the range of $\chi_{K}$ is finite-dimensional.

${ }^{9}$ The condition that $A$ is trace class on compact sets is satisfied whenever $A$ is in $\mathcal{B}_{1}^{\tau}$, as proved in Lemma 2.3.
} 
2. Let $A$ be a periodic and bounded operator acting on $\mathcal{H}$. Denoting by

$$
\mathcal{U}_{\mathrm{BF}} A \mathcal{U}_{\mathrm{BF}}^{-1}=\int_{\mathbb{R}^{d}}^{\oplus} \mathrm{d} k A(k)
$$

its Bloch-Floquet-Zak decomposition, assume that $A(k)$ is trace class and that $\operatorname{Tr}_{\mathcal{H}_{\mathrm{f}}}(|A(k)|)<C$ for all $k \in \mathbb{B}^{d}$. Then

$$
\operatorname{Tr}\left(\chi_{1} A \chi_{1}\right)=\frac{1}{\left|\mathbb{B}^{d}\right|} \int_{\mathbb{B}^{d}} \mathrm{~d} k \operatorname{Tr}_{\mathcal{H}_{\mathrm{f}}}(A(k)) .
$$

Proof. 1. In view of the decomposition (2.8) and the hypotheses on $A$, one has

$$
\operatorname{Tr}\left(\chi_{L} A \chi_{L}\right)=\sum_{\gamma \in \Gamma \cap \mathcal{C}_{L}} \operatorname{Tr}\left(T_{\gamma} \chi_{1} T_{\gamma}^{*} A T_{\gamma} \chi_{1} T_{\gamma}^{*}\right)=\sum_{\gamma \in \Gamma \cap \mathcal{C}_{L}} \operatorname{Tr}\left(\chi_{1} A \chi_{1}\right) .
$$

Since $\left|\mathcal{C}_{L}\right|=L^{d}\left|\mathcal{C}_{1}\right|=\operatorname{card}\left(\Gamma \cap \mathcal{C}_{L}\right)\left|\mathcal{C}_{1}\right|$ for every $L \in 2 \mathbb{N}+1$, one obtains

$$
\lim _{\substack{L \rightarrow \infty \mathbb{N}+1 \\ L \rightarrow}} \frac{1}{\left|\mathcal{C}_{L}\right|} \operatorname{Tr}\left(\chi_{L} A \chi_{L}\right)=\frac{1}{\left|\mathcal{C}_{1}\right|} \operatorname{Tr}\left(\chi_{1} A \chi_{1}\right)
$$

2. This is proved e.g. in [51, Lemma 3].

In the following result, we introduce a class of operators which are not necessarily in $\mathcal{B}_{1}^{\tau}$, but have finite trace per unit volume.

Proposition 2.5. Let $A$ be periodic and trace class on compact sets ${ }^{(9)}$. Then

1. the operator $X_{j} A$ for $j \in\{1, \ldots, d\}$ has finite trace per unit volume and

$$
\tau\left(X_{j} A\right)=\frac{1}{\left|\mathcal{C}_{1}\right|} \operatorname{Tr}\left(\chi_{1} X_{j} A \chi_{1}\right) .
$$

2. If, in addition $\tau(A)=0$, then $\tau\left(X_{j} A\right)$ does not depend on the exhaustion $^{10} \mathcal{C}_{L} \nearrow \mathcal{X}$ set in Definition 2.2 and on the choice of the origin, in the sense that

$$
\tau\left(\left(X_{j}+\alpha\right) A\right)=\tau\left(X_{j} A\right) \quad \forall \alpha \in \mathbb{R} .
$$

Proof. 1. Since $\chi_{L} X_{j} \chi_{L}$ is bounded for every $L \in 2 \mathbb{N}+1$ and $A$ is trace class on compact sets by hypothesis, we have that $\chi_{L} X_{j} A \chi_{L}=\chi_{L} X_{j} \chi_{L} A \chi_{L}$ is trace class. Therefore, in view of the decomposition (2.8), one has that $\operatorname{Tr}\left(\chi_{L} X_{j} A \chi_{L}\right)=\sum_{\gamma \in \Gamma \cap \mathcal{C}_{L}} \operatorname{Tr}\left(T_{\gamma} \chi_{1} T_{\gamma}^{*} X_{j} A T_{\gamma} \chi_{1} T_{\gamma}^{*}\right)=\sum_{\gamma \in \Gamma \cap \mathcal{C}_{L}} \operatorname{Tr}\left(\chi_{1} T_{\gamma}^{*} X_{j} A T_{\gamma} \chi_{1}\right)$.

Using that $A$ is periodic, that $\left[T_{\gamma}, X_{j}\right]=-\gamma_{j} T_{\gamma}$, and the result from Proposition 2.41, we obtain that

$$
\operatorname{Tr}\left(\chi_{1} T_{\gamma}^{*} X_{j} A T_{\gamma} \chi_{1}\right)=\operatorname{Tr}\left(\chi_{1}\left(X_{j}+\gamma_{j}\right) A \chi_{1}\right)=\operatorname{Tr}\left(\chi_{1} X_{j} A \chi_{1}\right)+\gamma_{j}\left|\mathcal{C}_{1}\right| \tau(A) .
$$

Consequently, we get that

$$
\frac{1}{L^{d}\left|\mathcal{C}_{1}\right|} \operatorname{Tr}\left(\chi_{L} X_{j} A \chi_{L}\right)=\frac{1}{\left|\mathcal{C}_{1}\right|} \operatorname{Tr}\left(\chi_{1} X_{j} A \chi_{1}\right)+\frac{\tau(A)}{L^{d}}\left(\sum_{\gamma \in \Gamma \cap \mathcal{C}_{L}} \gamma_{j}\right) \text {. }
$$

\footnotetext{
${ }^{10}$ Notice that this particular choice of the exhaustion $\mathcal{C}_{L} \nearrow \mathcal{X}$ is such that $\mathcal{C}_{L} \cap \Gamma$ is symmetric with respect to the involution $x \mapsto-x$.
} 
Since both $\gamma$ and $-\gamma$ are in $\Gamma \cap \mathcal{C}_{L}$ for all $L \in 2 \mathbb{N}+1$, the sum in brackets on the right-hand side of the above vanishes, and the thesis follows immediately.

2. The statement follows from (2.16) and the hypothesis $\tau(A)=0$.

A property which will be fundamental for all the following analysis is the conditional cyclicity of the trace per unit volume. We state it in the following lemma, whose proof can be found in [12, Lemma 3.22].

Lemma 2.6 (Conditional cyclicity of the trace per unit volume). If $A \in \mathcal{B}_{1}^{\tau}$ and $B \in \mathcal{B}_{\infty}^{\tau}$, then $\tau(A B)=\tau(B A)$.

The trace per unit volume is defined in (2.9) through a specific choice of the cell $\mathcal{C}_{L}$, which in turn depends via (2.7) on the choice of a particular linear basis $\left\{a_{1}, \ldots, a_{d}\right\}$ for $\Gamma$. The term unit cell consistency refers to the requirement that physically relevant quantities are independent of the latter choice. Precisely, one considers a different linear basis $\left\{\tilde{a}_{1}, \ldots, \tilde{a}_{d}\right\}$ for $\Gamma$ and the corresponding cell, defined by

$$
\widetilde{\mathcal{C}}_{L}:=\left\{x \in \mathcal{X}: x=\sum_{j=1}^{d} \alpha_{j} \tilde{a}_{j} \text { with }\left|\alpha_{j}\right| \leq L / 2 \forall j \in\{1, \ldots, d\}\right\},
$$

and sets $\widetilde{\chi}_{L}:=\chi_{\widetilde{\mathcal{C}}_{L}}$. Denoting by $\tau(\cdot)$ and $\widetilde{\tau}(\cdot)$, respectively, the trace per unit volume induced by the choice of the primitive cells $\mathcal{C}_{1}$ and $\widetilde{\mathcal{C}}_{1}$, we prove in Proposition A.2.1 that for any periodic operator $A$, which is trace class on compact sets, one has that

$$
\tau(A)=\widetilde{\tau}(A) .
$$

When a contribution to the transport coefficient is in the form $\tau\left(X_{i} A\right)$ for a periodic operator $A$, as in formula (1.15), a more careful analysis is needed, as discussed at the end of Sect. 5.1 and in Appendix A.

\section{The Unperturbed Model}

Our goal is to study the linear response of a crystalline system to the application of an external electric field of small intensity. Before considering the perturbed system, we state our assumptions on the unperturbed one.

Assumption 3.1. We assume the following:

$\left(\mathrm{H}_{1}\right)$ the Hamiltonian $H_{0}$ of the unperturbed system is a self-adjoint periodic operator on $\mathcal{H}$, bounded from below, such that in Bloch-Floquet-Zak representation its fibration

$$
H_{0}: \mathbb{R}^{d} \rightarrow \mathcal{L}\left(\mathcal{D}_{\mathrm{f}}, \mathcal{H}_{\mathrm{f}}\right), \quad k \mapsto H_{0}(k),
$$

is a smooth equivariant map taking values in the self-adjoint operators with dense domain $\mathcal{D}_{\mathrm{f}} \subset \mathcal{H}_{\mathrm{f}}$, such that $\varrho\left(\gamma^{*}\right): \mathcal{D}_{\mathrm{f}} \rightarrow \mathcal{D}_{\mathrm{f}}$ for every $\gamma^{*} \in$ $\Gamma^{*}$ (compare $\left.(2.5)\right)$. Here $\mathcal{L}\left(\mathcal{D}_{\mathrm{f}}, \mathcal{H}_{\mathrm{f}}\right)$ denotes the space of bounded linear operators from $\mathcal{D}_{\mathrm{f}}$, equipped with the graph norm of $H_{0}(0)$ denoted by $\|\cdot\|_{\mathcal{D}_{\mathrm{f}}}{ }^{11}$, to $\mathcal{H}_{\mathrm{f}} \simeq L^{2}\left(\mathcal{C}_{1}\right) \otimes \mathbb{C}^{N}$

\footnotetext{
${ }^{11}$ From now on, $\mathcal{D}_{\mathrm{f}}$ is understood to be equipped with the norm $\|\cdot\|_{\mathcal{D}_{\mathrm{f}}}$.
} 
$\left(\mathrm{H}_{2}\right)$ let $\mu \in \mathbb{R}$ (Fermi energy) be in a spectral gap ${ }^{12}$ of $H_{0}$. We denote by $\Pi_{0}=\chi_{(-\infty, \mu)}\left(H_{0}\right)$ the corresponding spectral projector (Fermi projector). We assume that its fibration $k \mapsto \Pi_{0}(k)$ takes values in the finite-rank projections on $\mathcal{H}_{\mathrm{f}}{ }^{13}$

We shortly discuss sufficient conditions implying that Assumption $\left(\mathrm{H}_{1}\right)$ holds true. As far as discrete models are concerned, $\mathcal{H}_{\mathrm{f}}$ is finite dimensional and $H_{0}(k)$ are self-adjoint matrices. The smoothness of the map $k \mapsto H_{0}(k)$ follows from the fact that the hopping amplitudes in the model $\left\{t_{\gamma}\right\}_{\gamma \in \Gamma}$ decay sufficiently fast as $|\gamma| \rightarrow \infty$. In all the most popular discrete models of topological insulators [25,33] the hopping amplitudes have finite range, namely $t_{\gamma}=0$ if $|\gamma|>R$ for some $R$, hence assumption $\left(\mathrm{H}_{1}\right)$ is automatically satisfied.

As for the continuum case $\mathcal{X}=\mathbb{R}^{d}$, we first consider a Bloch-Landau operator in the form

$$
H_{0}=\frac{1}{2}\left(-\mathrm{i} \nabla-\frac{1}{c} A\right)^{2}+V_{\Gamma},
$$

acting in $L^{2}\left(\mathbb{R}^{d}\right)$, where $A$ and $V_{\Gamma}$ are the magnetic and electrostatic potentials, respectively (the charge Q of the particle is reabsorbed in $A$ and in $V$ ). For the sake of simplicity, we consider only $d \leq 3$ and we ignore the "spin space" $\mathbb{C}^{N}$, but similar results holds true if, for example, $V_{\Gamma}$ is matrix-valued and acts non-trivially on these degrees of freedom. With the help of Kato's theory [35], and arguing as in [45] on the basis [11], it is not difficult to prove that, if $A=A_{\Gamma}$ is $\Gamma$-periodic, and $\mathcal{C}_{1}$ denotes the fundamental cell of the lattice, then for the validity of $\left(\mathrm{H}_{1}\right)$ it is sufficient to assume either of the following two sets of hypotheses:

(A) $A \in L^{\infty}\left(\mathcal{C}_{1}, \mathbb{R}^{2}\right)$ when $d=2$ or $A \in L^{4}\left(\mathcal{C}_{1}, \mathbb{R}^{3}\right)$ when $d=3$, and $\operatorname{div} A, V_{\Gamma} \in L_{\mathrm{loc}}^{2}\left(\mathbb{R}^{d}\right)$ when $d \in\{2,3\}$;

(B) $A \in L^{r}\left(\mathcal{C}_{1}, \mathbb{R}^{2}\right)$ with $r>2$ and $V_{\Gamma} \in L^{p}\left(\mathcal{C}_{1}\right)$ with $p>1$ when $d=2$, or $A \in L^{3}\left(\mathcal{C}_{1}, \mathbb{R}^{3}\right)$ and $V_{\Gamma} \in L^{3 / 2}\left(\mathcal{C}_{1}\right)$ when $d=3$.

If instead $A=A_{b}$ is a linear potential inducing a constant uniform magnetic field, it is enough to assume that $V_{\Gamma}$ is infinitesimally form bounded with respect to $-\Delta$ on $\mathcal{H}_{\mathrm{f}}$, and that the magnetic flux per unit cell is a rational multiple of the magnetic flux quantum. As a further example, we consider the Hamiltonian

$$
H_{0}=\frac{1}{2} \mathbf{p}^{2} \otimes \mathbb{1}_{\mathbb{C}^{2}}+\left(E_{1} p_{2}-E_{2} p_{1}\right) \otimes s_{z}+E_{3}\left(p_{1} \otimes s_{y}-p_{2} \otimes s_{x}\right),
$$

acting on $L^{2}\left(\mathbb{R}^{2}\right) \otimes \mathbb{C}^{2}$. In the above, $\mathbf{p} \equiv\left(p_{1}, p_{2}\right)=-\mathrm{i} \nabla$ denotes the momentum operator, $\mathbf{E} \equiv\left(E_{1}, E_{2}, E_{3}\right)$ is a constant vector (which we interpret as a constant electric field), and $\mathbf{s} \equiv\left(s_{x}, s_{y}, s_{z}\right)$ denotes the vector of spin matrices

\footnotetext{
${ }^{12}$ In the following, when we refer to "the" spectral gap of $H_{0}$, we will refer to this specific gap.

${ }^{13}$ From the smoothness assumption $\left(\mathrm{H}_{1}\right)$, it follows that $\operatorname{Rank}\left(\Pi_{0}(k)\right)=m \in \mathbb{N} \cup\{+\infty\}$ is independent of $k$. Therefore, in view of the fact that $\Pi_{0}$ is an orthogonal projection, $m<+\infty$ is equivalent to the assumption $\Pi_{0} \in \mathcal{B}_{1}^{\tau}$.
} 
(half of the Pauli matrices). Thus, the first term in $H_{0}$ represents the kinetic energy, the second one a spin-orbit coupling, and the third one is a Rashba term: This Hamiltonian represents a continuum analogue of the Kane-Mele Hamiltonian proposed in [33]. One can argue that the above operator can be fibered via Bloch-Floquet-Zak transform leading to a family of fiber Hamiltonians $H_{0}(k)$ as in Assumption $\left(\mathrm{H}_{1}\right)$. Moreover, since

$\left[H_{0}, S\right]=\left[H_{0}^{\mathrm{R}}, S\right]=E_{3}\left(p_{1} \otimes\left[s_{y}, s_{z}\right]-p_{2} \otimes\left[s_{x}, s_{z}\right]\right)=\mathrm{i} E_{3}\left(p_{1} \otimes s_{x}+p_{2} \otimes s_{y}\right)$, and since the momentum operator is relatively bounded with respect to the Laplacian, one can see that $\left[H_{0}, S\right]$ is relatively bounded with respect to $H_{0}$, and hence the assumptions on $S$ (compare Definition 5.1) are satisfied as well.

The following spaces of operators and functions turn out to be useful for our analysis.

Definition 3.2. Let $\mathcal{H}_{1}, \mathcal{H}_{2} \in\left\{\mathcal{D}_{\mathrm{f}}, \mathcal{H}_{\mathrm{f}}\right\}$. We denote by $\mathcal{L}\left(\mathcal{H}_{1}, \mathcal{H}_{2}\right)$ the space of bounded linear operators from $\mathcal{H}_{1}$ to $\mathcal{H}_{2}$ and by $\mathcal{L}\left(\mathcal{H}_{1}\right):=\mathcal{L}\left(\mathcal{H}_{1}, \mathcal{H}_{1}\right)$. We define

$\mathcal{P}\left(\mathcal{H}_{1}, \mathcal{H}_{2}\right):=\left\{\Gamma-\right.$ periodic $A$ with smooth fibration $\left.\mathbb{R}^{d} \rightarrow \mathcal{L}\left(\mathcal{H}_{1}, \mathcal{H}_{2}\right), k \mapsto A(k)\right\}$

equipped with the norm $\|A\|_{\mathcal{P}\left(\mathcal{H}_{1}, \mathcal{H}_{2}\right)}:=\max _{k \in \mathbb{B}^{d}}\|A(k)\|_{\mathcal{L}\left(\mathcal{H}_{1}, \mathcal{H}_{2}\right)}$. We also set $\mathcal{P}\left(\mathcal{H}_{1}\right):=\mathcal{P}\left(\mathcal{H}_{1}, \mathcal{H}_{1}\right)$.

Since the Fréchet derivative follows the usual rules of the differential calculus, we have that $\mathcal{P}\left(\mathcal{H}_{1}, \mathcal{H}_{2}\right)$ is a linear space, $\mathcal{P}\left(\mathcal{H}_{\mathrm{f}}\right), \mathcal{P}\left(\mathcal{D}_{\mathrm{f}}\right)$ and $\mathcal{P}\left(\mathcal{H}_{\mathrm{f}}, \mathcal{D}_{\mathrm{f}}\right)$ are normed algebras, and e.g. for $A \in \mathcal{P}\left(\mathcal{H}_{\mathrm{f}}, \mathcal{D}_{\mathrm{f}}\right)$ and $B \in \mathcal{P}\left(\mathcal{H}_{\mathrm{f}}\right)$ we have

$$
A B \in \mathcal{P}\left(\mathcal{H}_{\mathrm{f}}, \mathcal{D}_{\mathrm{f}}\right) \text { with }\|A B\|_{\mathcal{P}\left(\mathcal{H}_{\mathrm{f}}, \mathcal{D}_{\mathrm{f}}\right)} \leq\|A\|_{\mathcal{P}\left(\mathcal{H}_{\mathrm{f}}, \mathcal{D}_{\mathrm{f}}\right)}\|B\|_{\mathcal{P}\left(\mathcal{H}_{\mathrm{f}}\right)} .
$$

Definition 3.3. Let $\mathcal{H}_{1} \in\left\{\mathcal{D}_{\mathrm{f}}, \mathcal{H}_{\mathrm{f}}\right\}$. We set

$$
C_{\varrho}^{\infty}\left(\mathbb{R}^{d}, \mathcal{H}_{1}\right):=\left\{\varphi \in \mathcal{H}_{\varrho} \text { such that } \varphi: \mathbb{R}^{d} \rightarrow \mathcal{H}_{1} \text { is smooth }\right\} .
$$

Notice that $C_{\varrho}^{\infty}\left(\mathbb{R}^{d}, \mathcal{H}_{\mathrm{f}}\right) \supset C_{\varrho}^{\infty}\left(\mathbb{R}^{d}, \mathcal{D}_{\mathrm{f}}\right), C_{\varrho}^{\infty}\left(\mathbb{R}^{d}, \mathcal{H}_{\mathrm{f}}\right)$ is dense in $\mathcal{H}_{\varrho}$ with respect to $\|\cdot\|_{\mathcal{H}_{\varrho}}$, and $C_{\varrho}^{\infty}\left(\mathbb{R}^{d}, \mathcal{D}_{\mathrm{f}}\right)$ is dense in $L_{\varrho}^{2}\left(\mathbb{R}^{d}, \mathcal{D}_{\mathrm{f}}\right)$ with respect to the norm on $\mathcal{H}_{\varrho}$ induced by the graph norm $\|\cdot\|_{\mathcal{D}_{\mathrm{f}}}$.

Since we are interested in computing $\left[A, X_{j}\right]$ where $A$ is in one of the above spaces of operators, the following proposition will be relevant. It states their invariance under the derivation $\overline{\left[\cdot, X_{j}\right]}$, where the overline denotes the operator closure.

Proposition 3.4. Let $\mathcal{H}_{1}, \mathcal{H}_{2} \in\left\{\mathcal{D}_{\mathrm{f}}, \mathcal{H}_{\mathrm{f}}\right\}$, and $A \in \mathcal{P}\left(\mathcal{H}_{1}, \mathcal{H}_{2}\right)$. Then, $\overline{\left[A, X_{j}\right]}$ is in $\mathcal{P}\left(\mathcal{H}_{1}, \mathcal{H}_{2}\right)$, and

$$
\overline{\left.\left[A, X_{j}\right]\right|_{\mathcal{U}_{\mathrm{BF}}^{-1} C_{\varrho}^{\infty}\left(\mathbb{R}^{d}, \mathcal{H}_{1}\right)}}(k)=-\mathrm{i} \partial_{k_{j}} A(k) \text { in } \mathcal{L}\left(\mathcal{H}_{1}, \mathcal{H}_{2}\right) .
$$

Proof. Notice that

$$
\left.\mathcal{U}_{\mathrm{BF}} X_{j} \mathcal{U}_{\mathrm{BF}}^{-1}\right|_{C_{\varrho}^{\infty}\left(\mathbb{R}^{d}, \mathcal{H}_{1}\right)}=\left.\mathrm{i} \partial_{k_{j}}\right|_{C_{\varrho}^{\infty}\left(\mathbb{R}^{d}, \mathcal{H}_{1}\right)},
$$


thus for every $\varphi \in C_{\varrho}^{\infty}\left(\mathbb{R}^{d}, \mathcal{H}_{1}\right)$ one has that

$$
\mathcal{U}_{\mathrm{BF}}\left[A, X_{j}\right] \mathcal{U}_{\mathrm{BF}}^{-1} \varphi=\left[\int_{\mathbb{R}^{d}}^{\oplus} \mathrm{d} k A(k), \mathrm{i} \partial_{k_{j}}\right] \varphi=-\mathrm{i} \int_{\mathbb{R}^{d}}^{\oplus} \mathrm{d} k \partial_{k_{j}} A(k) \varphi .
$$

Since $\mathcal{U}_{\mathrm{BF}} A \mathcal{U}_{\mathrm{BF}}^{-1} C_{\varrho}^{\infty}\left(\mathbb{R}^{d}, \mathcal{H}_{1}\right) \subset C_{\varrho}^{\infty}\left(\mathbb{R}^{d}, \mathcal{H}_{2}\right)($ see $[35$, III- $\S 3.1$, Problem (3.11)]), the commutator appearing on the right-hand side of the first equality is densely defined on $C_{\varrho}^{\infty}\left(\mathbb{R}^{d}, \mathcal{H}_{1}\right)$ and so by unitary conjugation the commutator on the left-hand side is densely defined as well. Thus, Lemma 2.1 implies that $\left[A, X_{j}\right]$ acting on $\mathcal{U}_{\mathrm{BF}}^{-1} C_{\varrho}^{\infty}\left(\mathbb{R}^{d}, \mathcal{H}_{1}\right)$ is periodic.

Observe that

$$
\left\|\partial_{k_{j}} A(k) f(k, \cdot)\right\|_{\mathcal{H}_{2}} \leq\left\|\partial_{k_{j}} A\right\|_{\mathcal{P}\left(\mathcal{H}_{1}, \mathcal{H}_{2}\right)}\|f(k, \cdot)\|_{\mathcal{H}_{1}}
$$

for every $f(k, \cdot) \in \mathcal{H}_{1}$. By (3.3) and (3.4), one obtains

$$
\left\|\left[A, X_{j}\right](k) \varphi(k, \cdot)\right\|_{\mathcal{H}_{2}} \leq\left\|\partial_{k_{j}} A\right\|_{\mathcal{P}\left(\mathcal{H}_{1}, \mathcal{H}_{2}\right)}\|\varphi(k, \cdot)\|_{\mathcal{H}_{1}}
$$

for all $\varphi \in C_{\varrho}^{\infty}\left(\mathbb{R}^{d}, \mathcal{H}_{1}\right)$. Therefore, as $\mathcal{H}_{2}$ is a Banach space, the extension principle implies the thesis.

Lemma 3.5. Under Assumption 3.1 we have that $\left(H_{0}-z \mathbb{1}\right)^{-1} \in \mathcal{P}\left(\mathcal{H}_{\mathrm{f}}, \mathcal{D}_{\mathrm{f}}\right)$ for every $z \in \rho\left(H_{0}\right)$, and that $\Pi_{0} \in \mathcal{P}\left(\mathcal{H}_{\mathrm{f}}, \mathcal{D}_{\mathrm{f}}\right)$.

Proof. The first claim is evident because of $\left(H_{0}-z \mathbb{1}\right)^{-1}(k)=\left(H_{0}(k)-z \mathbb{1}\right)^{-1}$. Since $\mathcal{D}_{\mathrm{f}}$ is a Banach space, the second one follows from Riesz's formula

$$
\Pi_{0}(k)=\frac{\mathrm{i}}{2 \pi} \oint_{C}\left(H_{0}(k)-z \mathbb{1}\right)^{-1} \mathrm{~d} z,
$$

where $C$ is a positively-oriented complex contour intersecting the real axis at the Fermi energy (so, in the gap) and below the bottom of the spectrum of $H_{0}$.

Corollary 3.6. Under Assumption 3.1 we have that

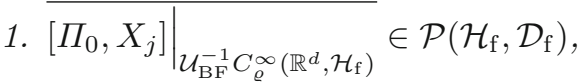

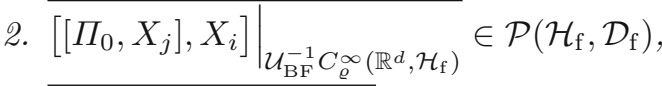

3. $\left.\left[H_{0}, X_{j}\right]\right|_{\mathcal{U}_{\mathrm{BF}}^{-1} C_{\varrho}^{\infty}\left(\mathbb{R}^{d}, \mathcal{D}_{\mathrm{f}}\right)} \in \mathcal{P}\left(\mathcal{D}_{\mathrm{f}}, \mathcal{H}_{\mathrm{f}}\right)$.

Proof. 1. By Lemma 3.5, one has that $\Pi_{0} \in \mathcal{P}\left(\mathcal{H}_{\mathrm{f}}, \mathcal{D}_{\mathrm{f}}\right)$. Proposition 3.4 implies the statement.

2. In view of Lemma 3.5, one has that $\Pi_{0} \in \mathcal{P}\left(\mathcal{H}_{\mathrm{f}}, \mathcal{D}_{\mathrm{f}}\right)$. Using an argument similar to the one presented in the proof of Proposition 3.4 one deduces the thesis.

3. Since by hypothesis $H_{0} \in \mathcal{P}\left(\mathcal{D}_{\mathrm{f}}, \mathcal{H}_{\mathrm{f}}\right)$, Proposition 3.4 concludes the proof. 
For the sake of readability, we introduce the concise notation

$$
\begin{aligned}
\overline{\left[\Pi_{0}, X_{j}\right]}:=\overline{\left.\left[\Pi_{0}, X_{j}\right]\right|_{\mathcal{U}_{\mathrm{BF}}^{-1} C_{\varrho}^{\infty}\left(\mathbb{R}^{d}, \mathcal{H}_{\mathrm{f}}\right)}}, \\
\overline{\left[\left[\Pi_{0}, X_{j}\right], X_{i}\right]}:=\overline{\left.\left[\left[\Pi_{0}, X_{j}\right], X_{i}\right]\right|_{\mathcal{U}_{\mathrm{BF}}^{-1} C_{\varrho}^{\infty}\left(\mathbb{R}^{d}, \mathcal{H}_{\mathrm{f}}\right)}} \\
\text { and } \overline{\left[H_{0}, X_{j}\right]}:=\overline{\left.\left[H_{0}, X_{j}\right]\right|_{\mathcal{U}_{\mathrm{BF}}^{-1} C_{\varrho}^{\infty}\left(\mathbb{R}^{d}, \mathcal{D}_{\mathrm{f}}\right)}} .
\end{aligned}
$$

\section{Non-equilibrium Almost-Stationary States}

Now that we have established the model for the unperturbed system, we consider the perturbed Hamiltonian

$$
H^{\varepsilon}:=H_{0}-\varepsilon X_{j},
$$

where $\varepsilon \in[0,1]$ is the strength of the external electric field pointing in the $j$-direction.

As discussed in Introduction, we are interested in the linear response of the system to such a perturbation when it starts initially in the zerotemperature equilibrium state $\Pi_{0}$. While it is clear that the perturbation given by the linear electric potential has the effect of driving the system out of equilibrium, the perturbation is slowly varying and thus acts locally merely as a shift in energy. Hence it is expected that the initial equilibrium state $\Pi_{0}$ changes continuously into a nearby non-equilibrium almost-stationary state (NEASS). A detailed discussion and justification of the concepts of NEASS can be found in $[41,68]$.

For the following construction of the NEASS in the present setting we only need to know that the operator $\Pi^{\varepsilon}$, representing the NEASS, is determined uniquely (up to terms of order $\mathcal{O}\left(\varepsilon^{M+1}\right)$ ) by the following two properties:

$\left(\mathrm{SA}_{1}\right) \Pi^{\varepsilon}=\mathrm{e}^{-\mathrm{i} \varepsilon \mathcal{S}^{\varepsilon}} \Pi_{0} \mathrm{e}^{\mathrm{i} \varepsilon \mathcal{S}^{\varepsilon}}$ for some bounded, periodic and self-adjoint operator $\mathcal{S}^{\varepsilon}$

$\left(\mathrm{SA}_{2}\right) \Pi^{\varepsilon}$ almost-commutes with the Hamiltonian $H^{\varepsilon}$, namely $\left[H^{\varepsilon}, \Pi^{\varepsilon}\right]=$ $\mathcal{O}\left(\varepsilon^{M+1}\right)$.

Here $\mathcal{O}\left(\varepsilon^{M+1}\right)$ is understood in the sense of the operator norm.

Proposition 4.1. Consider the Hamiltonian $H^{\varepsilon}=H_{0}-\varepsilon X_{j}$ with $H_{0}$ satisfying Assumption 3.1 .

1. Let $\mathcal{S}:=\mathrm{i} \mathcal{I}\left(\left[\overline{\left[X_{j}, \Pi_{0}\right]}, \Pi_{0}\right]\right)$, where

$$
\mathcal{I}(A):=\frac{\mathrm{i}}{2 \pi} \oint_{C} \mathrm{~d} z\left(H_{0}-z \mathbb{1}\right)^{-1}\left[A, \Pi_{0}\right]\left(H_{0}-z \mathbb{1}\right)^{-1},
$$

with $C$ a positively-oriented contour in the complex energy plane enclosing the part of the spectrum of $H_{0}$ below the gap. Then $\mathcal{S}$ is in $\mathcal{P}\left(\mathcal{H}_{\mathrm{f}}, \mathcal{D}_{\mathrm{f}}\right)$ and is self-adjoint. 
2. Let $\Pi^{\varepsilon}:=\mathrm{e}^{-\mathrm{i} \varepsilon \mathcal{S}} \Pi_{0} \mathrm{e}^{\mathrm{i} \varepsilon \mathcal{S}}$. Then, $\Pi^{\varepsilon}=\Pi_{0}+\varepsilon \Pi_{1}+\varepsilon^{2} \Pi_{r}^{\varepsilon}$, where both $\Pi_{1}=\mathcal{I}\left(\overline{\left[X_{j}, \Pi_{0}\right]}\right)$ and $\Pi_{r}^{\varepsilon}$ are in $\mathcal{P}\left(\mathcal{H}_{\mathrm{f}}, \mathcal{D}_{\mathrm{f}}\right)$, and the map $[0,1] \ni \varepsilon \mapsto$ $\Pi_{r}^{\varepsilon} \in \mathcal{P}\left(\mathcal{H}_{\mathrm{f}}, \mathcal{D}_{\mathrm{f}}\right)$ is bounded. Moreover, $\overline{\left[H^{\varepsilon}, \Pi^{\varepsilon}\right]}=\varepsilon^{2} R^{\varepsilon}$, where $R^{\varepsilon}$ is in $\mathcal{P}\left(\mathcal{H}_{\mathrm{f}}\right)$ and the map $[0,1] \ni \varepsilon \mapsto R^{\varepsilon} \in \mathcal{P}\left(\mathcal{H}_{\mathrm{f}}\right)$ is bounded.

We postpone the proof of the above proposition to Sect. 6.3. It is already clear from the statement that the map $\mathcal{I}(\cdot)$ plays a crucial role: Its properties are summarized in Sect. 6.2, where we recall in particular the well-known fact from perturbation theory that $\mathcal{I}(A)$ is the unique solution to the equation $\left[H_{0}, \mathcal{I}(A)\right]=A$ whenever $A$ is off-diagonal in the orthogonal decomposition induced by $\Pi_{0}$.

\section{Results on the $S$-conductivity}

As stated in the previous sections, we want to investigate quantum $S$-currents induced by the perturbation given by an external electric field and compute their $S$-conductivities as linear response coefficients. To fix the ideas, the reader can think of the case $S=\mathbb{1}_{\mathcal{H}}$ (which corresponds physically to the charge current, in appropriate units, e.g. in quantum Hall systems) or to $S=\mathbb{1}_{L^{2}(\mathcal{X})} \otimes$ $s_{z}$, where $s_{z}=\sigma_{z} / 2$ is half of the third Pauli matrix (which corresponds to the spin current e.g. in quantum spin Hall systems).

Definition 5.1 ( $S$-current and $S$-conductivity). Let $S=\mathbb{1}_{L^{2}(\mathcal{X})} \otimes s$ be a selfadjoint operator on $\mathcal{H}=L^{2}(\mathcal{X}) \otimes \mathbb{C}^{N}$. Furthermore, assume that $S$ is periodic ${ }^{14}$ and its fibration $\mathbb{1}_{L^{2}\left(\mathcal{C}_{1}\right)} \otimes s$ is in $\mathcal{L}\left(\mathcal{D}_{\mathrm{f}}\right)$.

The conventional and the proper $S$-current operator are defined, respectively, as

$$
\begin{aligned}
& J_{\text {conv }, i}^{S}:=\frac{1}{2}\left(\mathrm{i} \overline{\left[H_{0}, X_{i}\right]} S+\mathrm{i} S \overline{\left[H_{0}, X_{i}\right]}\right) \\
& J_{\text {prop }, i}^{S}:=\mathrm{i} \overline{\left[H_{0}, X_{i}\right]} S+\mathrm{i} X_{i}\left[H_{0}, S\right]
\end{aligned}
$$

where $H_{0}$ satisfies Assumption 3.1. The conventional and proper $S$-conductivity are defined, respectively, as

$$
\operatorname{Re} \tau\left(J_{\text {conv } / \text { prop }, i}^{S} \Pi^{\varepsilon}\right)-\operatorname{Re} \tau\left(J_{\text {conv } / \text { prop }, i}^{S} \Pi_{0}\right)=: \varepsilon \sigma_{\text {conv } / \text { prop }, i j}^{S}+o(\varepsilon) .
$$

Since $\Pi^{\varepsilon}=\Pi_{0}+\varepsilon \Pi_{1}+\varepsilon^{2} \Pi_{r}^{\varepsilon}$ by Proposition 4.1.2, we have that

$$
\operatorname{Re} \tau\left(J_{\mathrm{prop}, i}^{S} \Pi^{\varepsilon}\right)-\operatorname{Re} \tau\left(J_{\mathrm{prop}, i}^{S} \Pi_{0}\right)=\varepsilon \operatorname{Re} \tau\left(J_{\mathrm{prop}, i}^{S} \Pi_{1}\right)+\varepsilon^{2} \operatorname{Re} \tau\left(J_{\mathrm{prop}, i}^{S} \Pi_{r}^{\varepsilon}\right) .
$$

In order to prove that $\operatorname{Re} \tau\left(J_{\text {prop }, i}^{S} \Pi_{1}\right)=\sigma_{\text {prop }, i j}^{S}$ according to (5.1), it suffices to show that all the traces per unit volume above are well defined and finite,

\footnotetext{
${ }^{14}$ Notice that this assumption is not automatically satisfied since the (magnetic) translation operators (see (2.2)) may act non-trivially on the factor $\mathbb{C}^{N}$. Obviously, for either the standard magnetic translations [71] or translation operators with a trivial action on the factor $\mathbb{C}^{N}$ the periodicity of $S$ is ensured.
} 
and that the term carrying a prefactor $\varepsilon^{2}$ is uniformly bounded in $\varepsilon$. While the control of the remainder term will be done in Sect. 6.4, we focus now on the linear response coefficient, namely $\operatorname{Re} \tau\left(J_{\text {prop }, i}^{S} \Pi_{1}\right)$.

\subsection{The Linear Response Coefficient}

In order to compute the linear response coefficient, we employ directly Definition 2.2 for $\tau\left(J_{\text {prop }, i}^{S} \Pi_{1}\right)$, and start by localizing this operator on the cell $\mathcal{C}_{L}$, defined in $(2.7)$, through the projection $\chi_{L}$ which multiplies by the characteristic function of $\mathcal{C}_{L}$. It is convenient to notice at this point the following

Remark 5.2. The range of $\mathcal{U}_{\mathrm{BF}} \chi_{L}$ is contained in $C_{\varrho}^{\infty}\left(\mathbb{R}^{d}, \mathcal{H}_{\mathrm{f}}\right)$ (compare Definition 3.3) for every $L>0$. Indeed, for all $f \in \mathcal{H}=L^{2}(\mathcal{X}) \otimes \mathbb{C}^{N}$ and all $r \in \mathbb{N}$, the function $\langle X\rangle^{r} \chi_{L} f$ is still in $\mathcal{H}$, where $(\langle X\rangle \psi)(x):=\left(1+|x|^{2}\right)^{1 / 2} \psi(x)$ for $\psi \in \mathcal{D}(\langle X\rangle)$. By standard Bloch-Floquet theory [45, Appendix A], this is equivalent to requiring that $\mathcal{U}_{\mathrm{BF}}\left(\chi_{L} f\right)$ is in the space $H_{\varrho}^{r}\left(\mathbb{R}^{d}, \mathcal{H}_{\mathrm{f}}\right)$ of $\varrho$-covariant maps $\varphi: \mathbb{R}^{d} \rightarrow \mathcal{H}_{\mathrm{f}}$ with Sobolev regularity $r$ : It is a classical result that the intersection of all these Sobolev spaces is contained in $C_{\varrho}^{\infty}\left(\mathbb{R}^{d}, \mathcal{H}_{\mathrm{f}}\right)$.

Notice that, since $\Pi_{1} \in \mathcal{P}\left(\mathcal{H}_{\mathrm{f}}, \mathcal{D}_{\mathrm{f}}\right)$, it maps $\mathcal{U}_{\mathrm{BF}}^{-1} C_{\varrho}^{\infty}\left(\mathbb{R}^{d}, \mathcal{H}_{\mathrm{f}}\right)$ to $\mathcal{U}_{\mathrm{BF}}^{-1}$ $C_{\varrho}^{\infty}\left(\mathbb{R}^{d}, \mathcal{D}_{\mathrm{f}}\right)$. Thus, we have that

$$
J_{\text {prop }, i}^{S} \Pi_{1} \chi_{L}=\mathrm{i}\left[H_{0}, S X_{i}\right] \Pi_{1} \chi_{L}
$$

and this allows for the following simple manipulations. Using the Leibniz rule, we obtain the following chain of equalities on $\operatorname{Ran}\left(\chi_{L}\right)$ :

$$
\mathrm{i}\left[H_{0}, S X_{i}\right] \Pi_{1}=\mathrm{i}\left[H_{0}, X_{i} S\right] \Pi_{1}=\mathrm{i}\left[H_{0}, X_{i}\right] S \Pi_{1}+\mathrm{i} X_{i}\left[H_{0}, S\right] \Pi_{1}=\mathrm{O}+X_{i} \mathrm{R},
$$

where the operators $\mathrm{O}$ and $\mathrm{R}$ have been defined in (1.12). We call $\mathrm{O}$ the $S$ orbital part and $X_{i} \mathrm{R}$ the $S$-rotation part of the operator related to linear response of the proper $S$-current $J_{\text {prop }, i}^{S}$. The latter terminology is due to the fact that, when $S$ is the spin operator and $\left[H_{0}, S\right] \neq 0$, the spin transport is the result of two contributions, that is, the $S$-orbital part coming from the center-of-mass drift and the $S$-rotation part due to the spin non-conservation. Notice that both $\mathrm{O}$ and $\mathrm{R}$ are periodic, instead obviously $X_{i} \mathrm{R}$ is not periodic and thus a more careful analysis of its trace per unit volume is required. We begin by handling the $S$-orbital part O. In view of the defining relation $\Pi_{1}=\mathcal{I}\left(\overline{\left[X_{j}, \Pi_{0}\right]}\right)$ (compare Propositions 4.1 and 6.3), we obtain on $\operatorname{Ran}\left(\chi_{L}\right)$ :

$$
\begin{aligned}
\mathrm{O} & =\mathrm{i}\left[H_{0}, X_{i}^{\mathrm{D}}\right] S \Pi_{1}+\mathrm{i}\left[H_{0}, X_{i}^{\mathrm{OD}}\right] S \Pi_{1} \\
& =\mathrm{E}_{1}+\mathrm{i}\left[H_{0}, X_{i}^{\mathrm{OD}} S \Pi_{1}\right]-\mathrm{i} X_{i}^{\mathrm{OD}}\left[H_{0}, S \Pi_{1}\right] \\
& =\mathrm{E}_{1}+\mathrm{E}_{2}-\mathrm{i} X_{i}^{\mathrm{OD}}\left[H_{0}, S\right] \Pi_{1}-\mathrm{i} X_{i}^{\mathrm{OD}} S\left[X_{j}, \Pi_{0}\right] \\
& =\sum_{\ell=1}^{3} \mathrm{E}_{\ell}-\mathrm{i}\left[\left[X_{i}, \Pi_{0}\right], \Pi_{0} S\left[X_{j}, \Pi_{0}\right]\right]+\mathrm{i} \Pi_{0}\left[\left[X_{i}, \Pi_{0}\right], S\left[X_{j}, \Pi_{0}\right]\right] \\
& =\sum_{\ell=1}^{4} \mathrm{E}_{\ell}+\mathrm{C},
\end{aligned}
$$


where we have defined the operators

$$
\begin{aligned}
& \mathrm{E}_{1}:=\mathrm{i}\left[H_{0}, X_{i}^{\mathrm{D}}\right] S \Pi_{1}, \quad \mathrm{E}_{2}:=\mathrm{i}\left[H_{0}, X_{i}^{\mathrm{OD}} S \Pi_{1}\right], \quad \mathrm{E}_{3}:=\mathrm{i} X_{i}^{\mathrm{OD}}\left[S, H_{0}\right] \Pi_{1}, \\
& \mathrm{E}_{4}:=\mathrm{i}\left[\left[X_{i}, \Pi_{0}\right], \Pi_{0} S\left[\Pi_{0}, X_{j}\right]\right] \quad \text { and } \quad \mathrm{C}:=\mathrm{i} \Pi_{0}\left[\left[X_{i}, \Pi_{0}\right], S\left[X_{j}, \Pi_{0}\right]\right] .
\end{aligned}
$$

We call $\mathrm{C}$ the Chern-like term and $\mathrm{E}_{\ell}$ the $\ell$-th extra or beyond-Chern-like term for $\ell \in\{1, \ldots, 4\}$. This terminology is motivated by the fact that whenever the spin is conserved, for $d=2, i=1$ and $j=2$ in quantum (spin) Hall systems the Chern-like term C corresponds to the (spin-)Chern number (see Remark 5.12). In general, whenever $\left[H_{0}, S\right]=0$, all extra terms have trace per unit volume zero (see Sect. 5.2) and obviously the $S$-rotation part vanishes.

In the following proposition, we analyze the trace per unit volume of the operators resulting from the previous algebraic manipulations.

Proposition 5.3. Under Assumption 3.1 and hypotheses on $S$ in Definition 5.1, we have that the Chern-like term $\mathrm{C}$, the extra terms $\mathrm{E}_{\ell}$ for any $\ell \in\{1, \ldots, 4\}$ and $X_{i} \mathrm{R}$, defined in (1.12) and (5.6) have finite traces per unit volume. Moreover, one has

$$
\tau(\mathrm{A})=\frac{1}{\left|\mathcal{C}_{1}\right|} \operatorname{Tr}\left(\chi_{1} \mathrm{~A} \chi_{1}\right), \text { for } \mathrm{A} \in\left\{\mathrm{C}, \mathrm{E}_{\ell}, X_{i} \mathrm{R}: \ell \in\{1, \ldots, 4\}, i \in\{1, \ldots, d\}\right\},
$$

and

$$
\begin{aligned}
\tau(\mathrm{C}) & =\mathrm{i} \tau\left(\Pi_{0}\left[\left[X_{i}, \Pi_{0}\right] S,\left[X_{j}, \Pi_{0}\right]\right]\right), \\
\tau\left(\mathrm{E}_{1}\right) & =\mathrm{i} \tau\left(\left[H_{0}, X_{i}^{\mathrm{D}}\right] S^{\mathrm{OD}} \Pi_{1}\right), \quad \tau\left(\mathrm{E}_{3}\right)=\mathrm{i} \tau\left(X_{i}^{\mathrm{OD}}\left[S, H_{0}\right] \Pi_{1}\right), \\
\tau\left(\mathrm{E}_{2}\right) & =0=\tau\left(\mathrm{E}_{4}\right),
\end{aligned}
$$

where the diagonal and off-diagonal parts of the above operators refer to the orthogonal decomposition induced by the Fermi projection $\Pi_{0}$.

The proof of the above proposition is postponed to Sect. 6.5.

We are going to prove that trace per unit volume of the operator $X_{i} \mathrm{R}$ is well defined and finite. In view of Proposition 2.5.2, it suffices to show that $\tau(\mathrm{R})$ is zero. The latter result is an immediate consequence of the following

Proposition 5.4. If $H_{0}$ satisfies Assumption 3.1, and $B$ is in $\mathcal{P}\left(\mathcal{H}_{\mathrm{f}}\right) \cap \mathcal{P}\left(\mathcal{D}_{\mathrm{f}}\right)$ (in particular, $B$ is a bounded periodic operator) the following holds:

1 .

$$
\tau\left(\mathrm{i}\left[H_{0}, B\right] \Pi_{1}\right)=\tau\left(\mathrm{i} \Pi_{0}\left[\left[B, \Pi_{0}\right],\left[X_{j}, \Pi_{0}\right]\right]\right) .
$$

2. If, in addition, $\left[B, X_{j}\right]=0$ then

$$
\tau\left(\mathrm{i}\left[H_{0}, B\right] \Pi_{1}\right)=0 .
$$

The above proposition, whose proof is deferred to Sect. 6.5, immediately implies the following 
Corollary 5.5. Under Assumption 3.1 and hypotheses on $S$ in Definition 5.1, we have that

$$
\tau(\mathrm{R})=\tau\left(\mathrm{i}\left[H_{0}, S\right] \Pi_{1}\right)=0
$$

We are now in position to state one of our main results.

Theorem 5.6. (General formula for the $S$-conductivity) Let $H^{\varepsilon}=H_{0}-\varepsilon X_{j}$ be acting in $L^{2}(\mathcal{X}) \otimes \mathbb{C}^{N}$, with $H_{0}$ and $\Pi_{0}$ as in Assumption 3.1. Let $\Pi^{\varepsilon}$ be the NEASS defined in Sect. 4. Consider the conventional (resp. proper) $S$ conductivity $\sigma_{\mathrm{conv}, i j}$ (resp. $\sigma_{\mathrm{prop}, i j}$ ) as in Definition 5.1. Then

$$
\sigma_{\text {prop }, i j}^{S}=\sigma_{\text {conv }, i j}^{S}+\sigma_{\text {rot }, i j}^{S},
$$

where

$$
\begin{aligned}
\sigma_{\text {conv }, i j}^{S}= & \frac{1}{\left|\mathcal{C}_{1}\right|} \operatorname{Re} \operatorname{Tr}\left(\chi_{1} \mathrm{i} \Pi_{0}\left[\left[X_{i}, \Pi_{0}\right] S,\left[X_{j}, \Pi_{0}\right]\right] \chi_{1}\right) \\
& +\frac{1}{\left|\mathcal{C}_{1}\right|} \operatorname{Re} \operatorname{Tr}\left(\chi_{1}\left(\mathrm{i}\left[H_{0}, X_{i}^{\mathrm{D}}\right] S^{\mathrm{OD}} \Pi_{1}+\mathrm{i} X_{i}^{\mathrm{OD}}\left[S, H_{0}\right] \Pi_{1}\right) \chi_{1}\right)
\end{aligned}
$$

and the rotation contribution to the proper $S$-conductivity is defined as

$$
\sigma_{\text {rot }, i j}^{S}=\operatorname{Re} \tau\left(\mathrm{i} X_{i}\left[H_{0}, S\right] \Pi_{1}\right)=\frac{1}{\left|\mathcal{C}_{1}\right|} \operatorname{Re} \operatorname{Tr}\left(\chi_{1} \mathrm{i} X_{i}\left[H_{0}, S\right] \Pi_{1} \chi_{1}\right) .
$$

Moreover, the trace per unit volume appearing in (5.13) does not depend on the particular exhaustion ${ }^{(10)} \mathcal{C}_{L} \nearrow \mathcal{X}$ chosen in Definition 2.2 and on the choice of the origin, in the sense that $\tau\left(X_{i} \mathrm{R}\right)=\tau\left(\left(X_{i}+\alpha\right) \mathrm{R}\right)$ for every $\alpha \in \mathbb{R}$.

The proof of the above theorem is postponed to Sect. 6 .

Remark 5.7. Some comments about the above result.

(i) Notice that one can rewrite the above formula for the proper $S$ conductivity $\sigma_{\text {prop }, i j}^{S}$, summing the two contributions $\sigma_{\text {conv }, i j}^{S}$ and $\sigma_{\text {rot }, i j}^{S}$ as follows:

$$
\begin{aligned}
\sigma_{\text {prop }, i j}^{S}= & \frac{1}{\left|\mathcal{C}_{1}\right|} \operatorname{Re} \operatorname{Tr}\left(\chi_{1}\left(\mathrm{i} \Pi_{0}\left[\left[X_{i}, \Pi_{0}\right] S,\left[X_{j}, \Pi_{0}\right]\right]+\mathrm{i}\left[H_{0}, X_{i}^{\mathrm{D}}\right] S^{\mathrm{OD}} \Pi_{1}\right) \chi_{1}\right) \\
& +\frac{1}{\left|\mathcal{C}_{1}\right|} \operatorname{Re} \operatorname{Tr}\left(\chi_{1} \mathrm{i} X_{i}^{\mathrm{D}}\left[H_{0}, S\right] \Pi_{1} \chi_{1}\right) .
\end{aligned}
$$

While (5.11) emphasizes the splitting between the drift contribution coming from the center-of-mass momentum and the one resulting from the spin rotation, the latter decomposition isolates the contribution coming from a periodic operator, in (5.14), and the one deriving from a nonperiodic operator, in (5.15).

(ii) The real part is needed in both (5.12) and (5.13), even if on $\operatorname{Ran}\left(\chi_{1}\right)$ one has that $J_{\text {prop }, i}^{S} \Pi^{\varepsilon}=\left(J_{\text {prop }, i}^{S}\right)^{*} \Pi^{\varepsilon}$. On the other hand, if $\left[H_{0}, S\right]=0$ then $\tau\left(J_{\operatorname{prop}, i}^{S} \Pi^{\varepsilon}\right)$ is automatically real. Moreover, for systems with a fermionic time-reversal symmetry $\Theta$ such that $\Theta S \Theta^{-1}=-S$, the number $\tau\left(J_{\text {prop }, i}^{S} \Pi_{1}\right)$ is real, so the real part is redundant. 
It is worth to investigate how the contributions to the proper $S$ conductivity, appearing in (5.11), behave under a change of primitive cell.

Proposition 5.8 (Unit Cell Consistency of the $S$-conductivity). Under the hypotheses of Theorem 5.6, we have that

1. $\sigma_{\text {conv }, i j}^{S}$ satisfies $U C C$.

2. If, in addition, the model enjoys a discrete rotational symmetry satisfying the hypotheses of Proposition A.3, then $\sigma_{\text {rot }, i j}^{S}$ satisfies UCC.

Proof. 1. Since all operators involved in the trace per unit volume computing $\sigma_{\text {conv }, i j}^{S}$ are periodic, Proposition A.2.1 implies the thesis. 2. By applying Proposition A.3.1 along with Proposition A.2.2, the conclusion follows.

The next proposition shows that in some discrete models with discrete rotational symmetry, one has that $\sigma_{\text {rot }, i j}^{S}=0$, and hence the choice between $J_{\text {prop }, i}^{S}$ and $J_{\text {conv }, i}^{S}$ becomes immaterial. Remarkably, the Kane-Mele model is in this class.

Proposition 5.9 (Equality of conventional and proper $S$-conductivity). Let $H_{0}$ be a discrete Hamiltonian with finite range hopping amplitudes and $S$ be as in Definition 5.1. Assume that the model satisfies the hypotheses of Proposition A.3 and Rank $\chi_{P_{\gamma}}=1$, where $\left\{P_{\gamma}\right\}_{\gamma \in I} \subset \mathcal{X}$ is the family of subsets defined in (A.1). Then

$$
\sigma_{\text {rot }, i j}^{S}=0 \quad \text { or equivalently } \quad \sigma_{\text {prop }, i j}^{S}=\sigma_{\text {conv }, i j}^{S} .
$$

Proof. By direct computation, since there exists $\lambda_{i, \gamma} \in \mathbb{R}$ such that $X_{i} \chi_{P_{\gamma}}=$ $\lambda_{i, \gamma} \chi_{P_{\gamma}}$, we have that

$$
\begin{aligned}
\left|\mathcal{C}_{1}\right| \sigma_{\text {rot }, i j}^{S} & =\operatorname{Re} \operatorname{Tr}\left(\chi_{1} \mathrm{i} X_{i}\left[H_{0}, S\right] \Pi_{1} \chi_{1}\right)=\sum_{\gamma \in I} \operatorname{Re} \operatorname{Tr}\left(\chi_{P_{\gamma}} X_{i} \mathrm{i}\left[H_{0}, S\right] \Pi_{1} \chi_{P_{\gamma}}\right) \\
& =\sum_{\gamma \in I} \lambda_{i, \gamma} \operatorname{Re} \operatorname{Tr}\left(\chi_{P_{\gamma}} \mathrm{i}\left[H_{0}, S\right] \Pi_{1} \chi_{P_{\gamma}}\right)=0
\end{aligned}
$$

because by Proposition A.3.1 $\operatorname{Tr}\left(\chi_{P_{\gamma}} \mathrm{i}\left[H_{0}, S\right] \Pi_{1} \chi_{P_{\gamma}}\right)=0$ for every $\gamma \in I$.

\subsection{When $S$ is (Approximately) Conserved}

The computation of the linear response coefficient $\sigma_{i j}^{S}$ simplifies considerably if we assume that $S$ is a conserved quantity of the system, namely that

$$
\left[H_{0}, S\right]=0 .
$$

Under this assumption, then $\left[\Pi_{0}, S\right]=0$ as well, since $\Pi_{0}$ is a spectral projection associated with $H_{0}$, and thus $S$ is diagonal in the decomposition induced by $\Pi_{0}$.

If (5.16) holds, then $J_{i}^{S}:=J_{\text {prop }, i}^{S}=J_{\text {conv }, i}^{S}=\mathrm{i}\left[\overline{\left[H_{0}, X_{i}\right]} S\right.$ is in $\mathcal{P}\left(\mathcal{D}_{\mathrm{f}}, \mathcal{H}_{\mathrm{f}}\right)$ by Corollary 3.6.3 and the hypothesis $S \in \mathcal{P}\left(\mathcal{D}_{\mathrm{f}}\right)$. Hence, since $\Pi^{\varepsilon} \in \mathcal{P}\left(\mathcal{H}_{\mathrm{f}}, \mathcal{D}_{\mathrm{f}}\right)$ by Proposition 4.1.2, we have that $J_{i}^{S} \Pi^{\varepsilon} \in \mathcal{P}\left(\mathcal{H}_{\mathrm{f}}\right)$ and furthermore applying Proposition 6.6.1, we deduce that $J_{i}^{S} \Pi^{\varepsilon}$ is $\tau$-class. Thus, the trace per unit 
volume of $J_{i}^{S} \Pi^{\varepsilon}$ is well defined and only the Chern-like term contributes to it. Indeed, by Proposition 5.3 the extra term $\mathrm{E}_{\ell}$ does not contribute for $\ell \in\{2,4\}$ and the next Lemma shows that $\tau\left(\mathrm{E}_{1}\right)=0$. Obviously, $\mathrm{E}_{3}=0=\mathrm{R}$ whenever (5.16) holds.

Lemma 5.10. Under Assumption 3.1 and the hypotheses on $S$ in Definition 5.1, assume further that $\left[H_{0}, S\right]=0$. Then,

$$
\tau\left(\mathrm{E}_{1}\right)=0 \quad \text { and } \quad \mathrm{E}_{3}=0=\mathrm{R}
$$

where $\mathrm{E}_{1}, \mathrm{E}_{3}$ and $\mathrm{R}$ are defined in (5.6) and (1.12).

The proof of Lemma 5.10 is also deferred to Sect. 6.5 , but it is easily seen to imply the following

Theorem 5.11. ( $S$-conductivity in the $S$ conserved case) Let $H^{\varepsilon}=H_{0}-\varepsilon X_{j}$ be acting in $L^{2}(\mathcal{X}) \otimes \mathbb{C}^{N}$, with $H_{0}$ and $\Pi_{0}$ as in Assumption 3.1. Let $\Pi^{\varepsilon}$ be the NEASS defined in Sect. 4 and $J_{i}^{S}$ be as in Definition 5.1. Assume moreover that $\left[H_{0}, S\right]=0$. Then, the $S$-conductivity is

$$
\begin{aligned}
\sigma_{i j}^{S} & =\frac{\mathrm{i}}{\left|\mathcal{C}_{1}\right|} \operatorname{Tr}\left(\chi_{1} S \Pi_{0}\left[\left[X_{i}, \Pi_{0}\right],\left[X_{j}, \Pi_{0}\right]\right] \chi_{1}\right) \\
& =\frac{\mathrm{i}}{(2 \pi)^{d}} \int_{\mathbb{B}^{d}} \mathrm{~d} k \operatorname{Tr}_{\mathcal{H}_{\mathrm{f}}}\left((\mathbb{1} \otimes s) \Pi_{0}(k)\left[\partial_{k_{j}} \Pi_{0}(k), \partial_{k_{i}} \Pi_{0}(k)\right]\right) .
\end{aligned}
$$

Proof. In view of Lemma 5.10, the extra terms $\mathrm{E}_{1}$ and $\mathrm{E}_{3}$, and the $S$-rotation part $\sigma_{\text {rot }, i j}^{S}$ do not contribute to the trace per unit volume of $J_{i}^{S} \Pi_{1}$. Therefore, using Proposition 2.4.2 we are going to compute the $k$-space representation of the trace of $\mathrm{C}_{\chi_{1}}=\mathrm{i} S \Pi_{0}\left[\left[X_{i}, \Pi_{0}\right],\left[X_{j}, \Pi_{0}\right]\right] \chi_{1}$. To this end, it suffices to notice that the fiber operator associated with $\overline{\left[X_{j}, \Pi_{0}\right]}$ in the Bloch-Floquet-Zak representation is given by $\mathrm{i} \partial_{k_{j}} \Pi_{0}(k)$ (Proposition 3.4) and that $\left|\mathcal{C}_{1}\right|\left|\mathbb{B}^{d}\right|=$ $(2 \pi)^{d}$.

This theorem applies in particular to the transverse charge current in quantum Hall systems $\left(S=\mathbb{1}_{\mathcal{H}}\right)$, and to the transverse spin current in quantum spin Hall systems $\left(S=\mathbb{1}_{L^{2}(\mathcal{X})} \otimes s_{z}\right)$ whenever the $z$-component of the spin is conserved. In particular, in the latter case we recover the formula for the spin conductivity proposed in $[16,63]$, which was derived assuming that the unperturbed Hamiltonian $H_{0}$ has an identically degenerate Bloch band, where the degeneracy comes from the spin degrees of freedom. So in that model effectively $\left(\Pi_{0} H_{0} \Pi_{0}\right)(k)=E_{0}(k) \mathbb{1}_{L^{2}(\mathcal{X})} \otimes \mathbb{1}_{\mathbb{C}^{2}}$, and (5.16) is in particular satisfied after projection to the relevant spectral subspace. Our argument used only (5.16) and no spectral assumption (other than the gap condition) on the Hamiltonian.

Remark 5.12 (Spin conductivity and spin-Chern number). Let $S=\mathbb{1}_{L^{2}(\mathcal{X})} \otimes s_{z}$ with $s_{z}$ a spin operator for non-integer spin $r$, i.e. with spectrum $\{-r,-r+$ $1, \ldots, r-1, r\}$, acting on $\mathbb{C}^{N}$ with $N=2 r+1$ (e.g. half the third Pauli matrix $\sigma_{z}$ for $r=\frac{1}{2}$ and $\left.N=2\right)$. Denote by $s_{z}=\sum_{\ell=0}^{2 r}(\ell-r) p_{\ell}$ its spectral decomposition. 
Then, the commutation relation $\left[\Pi_{0}, S\right]=0$ implies that $\Pi_{0}$ admits a splitting in the decomposition induced by $S$ :

$$
\Pi_{0}=\sum_{\ell=0}^{2 r} \Pi_{0}^{(\ell)}:=\sum_{\ell=0}^{2 r} \Pi_{0}\left(\mathbb{1} \otimes p_{\ell}\right) .
$$

The formula for the $S$-conductivity $\sigma_{i j}^{S}$ in Theorem 5.11 simplifies then to

$$
\begin{aligned}
\sigma_{i j}^{S} & =\frac{\mathrm{i}}{\left|\mathcal{C}_{1}\right|} \sum_{\ell=0}^{2 r}(\ell-r) \operatorname{Tr}\left(\chi_{1} \Pi_{0}^{(\ell)}\left[\left[X_{i}, \Pi_{0}^{(\ell)}\right],\left[X_{j}, \Pi_{0}^{(\ell)}\right]\right] \chi_{1}\right) \\
& =: \frac{1}{(2 \pi)^{d-1}} S-\operatorname{Chern}\left(\Pi_{0}\right)_{i j} .
\end{aligned}
$$

This spin-Chern number $S$-Chern $\left(\Pi_{0}\right)_{i j}$, proposed in [61] and intrinsically defined in [56], is in general a half integer. ${ }^{15}$ It becomes an integer if the system enjoys time-reversal symmetry. Even in time-reversal invariant systems it can be different from zero while the Chern number

$$
\operatorname{Chern}\left(\Pi_{0}\right):=\frac{\mathrm{i}(2 \pi)^{d-1}}{\left|\mathcal{C}_{1}\right|} \operatorname{Tr}\left(\chi_{1} \Pi_{0}\left[\left[X_{i}, \Pi_{0}\right],\left[X_{j}, \Pi_{0}\right]\right] \chi_{1}\right)
$$

necessarily vanishes. Our approach to spin transport shows then that for $d=$ 2 the bulk spin Hall conductivity (measured in units of $\frac{e}{2 \pi} \equiv \frac{1}{2 \pi}$ ) equals the spin-Chern number, as long as $\left[H_{0}, S\right]=0$. On the other hand, when $S=\mathbb{1}_{L^{2}(\mathcal{X})} \otimes \mathbb{1}_{\mathbb{C}^{N}}$ and $d=2$ the integral in Theorem 5.11 computes, up to a factor $1 / 2 \pi$, the Chern number $\operatorname{Chern}\left(\Pi_{0}\right)$ of the family of projections $\left\{\Pi_{0}(k)\right\}_{k \in \mathbb{R}^{2}}$, implying the quantization of the Hall conductivity measured in units of $\frac{e^{2}}{h} \equiv \frac{1}{2 \pi}$ (see [22] and references therein).

Abstracting from the previous remark, we consider now any operator in the form $S=\mathbb{1}_{L^{2}(\mathcal{X})} \otimes s$, with $s$ as in Definition 5.1. If $S$ is approximately conserved, i.e. if $\lambda:=\left\|\left[H_{0}, S\right]\right\|_{\mathcal{P}\left(\mathcal{D}_{\mathrm{f}}, \mathcal{H}_{\mathrm{f}}\right)}$ is sufficiently small, then one can still define a spin-Chern number related to $\Pi_{0}[56,58]$ and the $S$-conductivity is still approximately given by the spin-Chern number. To see this, let $s=$ $\sum_{\ell=1}^{k} s_{\ell} p_{\ell}$ be the spectral representation of $s$ (we need no assumptions on the spectrum of $s$ here $), \widetilde{H}_{0}:=\sum_{\ell=1}^{k}\left(\mathbb{1} \otimes p_{\ell}\right) H_{0}\left(\mathbb{1} \otimes p_{\ell}\right) \quad$ and $\quad V:=\left(H_{0}-\right.$ $\left.\widetilde{H}_{0}\right)=\sum_{\ell_{1} \neq \ell_{2}}\left(\mathbb{1} \otimes p_{\ell_{1}}\right) H_{0}\left(\mathbb{1} \otimes p_{\ell_{2}}\right)$. Then, $H_{0}=\widetilde{H}_{0}+V$, where $\left[\widetilde{H}_{0}, S\right]=0$ and $\|V\|_{\mathcal{P}\left(\mathcal{D}_{\mathrm{f}}, \mathcal{H}_{\mathrm{f}}\right)}=\left\|\sum_{\ell_{1} \neq \ell_{2}}\left(\mathbb{1} \otimes p_{\ell_{1}}\right)\left[H_{0},\left(\mathbb{1} \otimes p_{\ell_{2}}\right)\right]\right\|_{\mathcal{P}\left(\mathcal{D}_{\mathrm{f}}, \mathcal{H}_{\mathrm{f}}\right)} \leq \lambda C_{s}$, with a constant $C_{s}$ that depends only on $S$.

The spin-conserving Hamiltonian $\widetilde{H}_{0}$ is $H_{0}$-bounded with relative bound $\lambda C_{s}$. For $\lambda<\frac{1}{C_{s}}, \widetilde{H}_{0}$ is thus self-adjoint on the domain of $H_{0}$, and for $\lambda$ small enough, by standard perturbation theory, the Fermi energy $\mu$ lies also in a gap of $\widetilde{H}_{0}$. Thus we can define the gapped Fermi projection $\widetilde{\Pi}_{0}:=\chi_{(-\infty, \mu]}\left(\widetilde{H}_{0}\right)$

\footnotetext{
${ }^{15}$ The normalization we use here agrees with [56] and with the most recent physics literature, but differs by a factor 2 from the original formula in [61].
} 
of $\widetilde{H}_{0}$ and, in analogy with Remark 5.12, its associated spin-Chern number. More precisely, let

$$
\widetilde{\Pi}_{0}^{(\ell)}:=\widetilde{\Pi}_{0}\left(\mathbb{1} \otimes p_{\ell}\right) \quad \text { and thus } \quad \sum_{\ell=1}^{k} \widetilde{\Pi}_{0}^{(\ell)}=\widetilde{\Pi}_{0} .
$$

It is straightforward to see that ${ }^{16} \widetilde{\Pi}_{0}^{(\ell)} \in \mathcal{P}\left(\mathcal{H}_{\mathrm{f}}, \mathcal{D}_{\mathrm{f}}\right) \cap \mathcal{B}_{1}^{\tau}$ and thus the Chern numbers

$$
\operatorname{Chern}\left(\widetilde{\Pi}_{0}^{(\ell)}\right)_{i j}:=\frac{\mathrm{i}(2 \pi)^{d-1}}{\left|\mathcal{C}_{1}\right|} \operatorname{Tr}\left(\chi_{1} \widetilde{\Pi}_{0}^{(\ell)}\left[\left[X_{i}, \widetilde{\Pi}_{0}^{(\ell)}\right],\left[X_{j}, \widetilde{\Pi}_{0}^{(\ell)}\right]\right] \chi_{1}\right) \in \mathbb{Z}
$$

are well defined and integer. The $S$-Chern number of $\Pi_{0}$ is finally defined as

$$
S-\operatorname{Chern}\left(\Pi_{0}\right)_{i j}:=\sum_{\ell=1}^{k} s_{\ell} \cdot \operatorname{Chern}\left(\widetilde{\Pi}_{0}^{(\ell)}\right)_{i j}
$$

We now show that the $S$-conductivity is given at leading order in $\lambda$ by $S-$ Chern $\left(\Pi_{0}\right)$, a result which coherently complements the robustness of edge spin currents proved by Schulz-Baldes [58].

To formulate such a perturbative statement precisely, we slightly change perspective and notation and introduce a $\lambda$-dependent family of Hamiltonians: Let $H_{0}$ satisfy Assumption 3.1 and $\left[H_{0}, S\right]=0$ and assume $V \in \mathcal{P}\left(\mathcal{D}_{\mathrm{f}}, \mathcal{H}_{\mathrm{f}}\right)$. Then, for $\lambda_{0}>0$ sufficiently small, it holds that $H_{\lambda}:=H_{0}+\lambda V$ is self-adjoint on the domain of $H_{0}$ and has a spectral gap at $\mu$ for each $\lambda \in\left[0, \lambda_{0}\right)$. As before we consider the gapped Fermi projection $\Pi_{\lambda}:=\chi_{(-\infty, \mu]}\left(H_{\lambda}\right)$ of $H_{\lambda}$, put $\Pi_{0}^{(\ell)}:=\Pi_{0}\left(\mathbb{1} \otimes p_{\ell}\right)$ and the associated Chern numbers Chern $\left(\Pi_{0}^{(\ell)}\right)_{i j}$. The $\lambda$-independent $S$-Chern number associated with $\Pi_{\lambda}$ is again $S$-Chern $\left(\Pi_{\lambda}\right)_{i j}:=$ $\sum_{\ell=1}^{k} s_{\ell} \cdot \operatorname{Chern}\left(\Pi_{0}^{(\ell)}\right)_{i j} \equiv S-\operatorname{Chern}\left(\Pi_{0}\right)_{i j}$.

Proposition 5.13. Let $H_{\lambda}=H_{0}+\lambda V$, be a perturbation of a spin-commuting Hamiltonian $H_{0}$ as defined above. Then, the $S$-conductivity $\sigma_{i j, \lambda}^{S}$ of $H_{\lambda}$ satisfies

$$
\sigma_{i j, \lambda}^{S}=S-\operatorname{Chern}\left(\Pi_{0}\right)_{i j}+\mathcal{O}(\lambda)
$$

Proof. By standard perturbation theory we obtain

$$
\begin{aligned}
& \left\|\Pi_{\lambda}-\Pi_{0}\right\|_{\mathcal{P}\left(\mathcal{H}_{\mathrm{f}}, \mathcal{D}_{\mathrm{f}}\right)}=\mathcal{O}(\lambda), \quad\left\|\left[\Pi_{\lambda}-\Pi_{0}, X_{i}\right]\right\|_{\mathcal{P}\left(\mathcal{H}_{\mathrm{f}}, \mathcal{D}_{\mathrm{f}}\right)}=\mathcal{O}(\lambda), \\
& \left\|\left[\Pi_{\lambda}, S\right]\right\|_{\mathcal{P}\left(\mathcal{H}_{\mathrm{f}}, \mathcal{D}_{\mathrm{f}}\right)}=\mathcal{O}(\lambda) .
\end{aligned}
$$

Observe that $\left\|\Pi_{\lambda}\right\|_{1, \tau}=\left\|\Pi_{0}\right\|_{1, \tau}$ using the smallness argument in ${ }^{(16)}$. Hence, starting from (5.14) and (5.15), we find

$$
\sigma_{i j, \lambda}^{S}=\frac{1}{\left|\mathcal{C}_{1}\right|} \operatorname{Re} \operatorname{Tr}\left(\chi_{1}\left(\mathrm{i} \Pi_{\lambda}\left[\left[X_{i}, \Pi_{\lambda}\right], S_{\lambda}^{\mathrm{D}}\left[X_{j}, \Pi_{\lambda}\right]\right]+\mathrm{i}\left[H_{\lambda}, X_{i, \lambda}^{\mathrm{D}}\right] S_{\lambda}^{\mathrm{OD}} \Pi_{1, \lambda}\right) \chi_{1}\right)
$$

\footnotetext{
${ }^{16} 4$ By choosing $\lambda$ small enough, one has that $\left\|\widetilde{\Pi}_{0}(k)-\Pi_{0}(k)\right\|<1$ and thus the two projections have the same rank for every $k$. Then, by the argument in the proof of Lemma 6.5 , it follows that $\widetilde{\Pi}_{0} \in \mathcal{B}_{1}^{\tau}$ and hence $\widetilde{\Pi}_{0}^{(\ell)} \in \mathcal{B}_{1}^{\tau}$ as well.
} 


$$
\begin{aligned}
& +\frac{1}{\left|\mathcal{C}_{1}\right|} \operatorname{Re} \operatorname{Tr}\left(\chi_{1} \mathrm{i} X_{i, \lambda}^{\mathrm{D}}\left[H_{\lambda}, S\right] \Pi_{1, \lambda} \chi_{1}\right) . \\
= & \frac{\mathrm{i}}{\left|\mathcal{C}_{1}\right|} \operatorname{Re} \operatorname{Tr}\left(\chi_{1} \Pi_{0}\left[\left[X_{i}, \Pi_{0}\right], S\left[X_{j}, \Pi_{0}\right]\right] \chi_{1}\right)+\mathcal{O}(\lambda) \\
= & \frac{\mathrm{i}}{\left|\mathcal{C}_{1}\right|} \sum_{\ell} s_{\ell} \operatorname{Tr}\left(\chi_{1} \Pi_{0}^{(\ell)}\left[\left[X_{i}, \Pi_{0}^{(\ell)}\right],\left[X_{j}, \Pi_{0}^{(\ell)}\right]\right] \chi_{1}\right)+\mathcal{O}(\lambda) \\
= & S-\operatorname{Chern}\left(\Pi_{0}\right)_{i j}+\mathcal{O}(\lambda) .
\end{aligned}
$$

In the second to last equality we used that $\mathbb{1} \otimes p_{\ell}$ commutes with $X_{i}$ and $\Pi_{0}$.

\section{Proofs}

\subsection{Diagonal and Off-Diagonal Operators}

The Fermi projection $\Pi_{0}$ of the unperturbed Hamiltonian $H_{0}$ clearly induces a decomposition of $L^{2}(\mathcal{X}) \otimes \mathbb{C}^{N}$ into $\operatorname{Ran} \Pi_{0} \oplus\left(\operatorname{Ran} \Pi_{0}\right)^{\perp}$. Correspondingly, operators acting in $L^{2}(\mathcal{X}) \otimes \mathbb{C}^{N}$ will admit a block decomposition. We review in this section some properties of this decomposition, heading toward the proof of a well-known formula from asymptotic perturbation theory which allows to invert the Liouvillian $\left[H_{0}, \cdot\right]$ acting on operators which only have off-diagonal blocks.

Definition 6.1 (Diagonal and off-diagonal parts). Given an operator $A$ and an orthogonal projection $\Pi$, i.e. $\Pi=\Pi^{*}=\Pi^{2}$, such that $A \Pi$ is densely defined $^{17}$, one defines its diagonal and off-diagonal parts as

$$
\begin{aligned}
A^{\mathrm{D}} & :=\Pi A \Pi+(\mathbb{1}-\Pi) A(\mathbb{1}-\Pi), \\
A^{\mathrm{OD}} & :=\Pi A(\mathbb{1}-\Pi)+(\mathbb{1}-\Pi) A \Pi,
\end{aligned}
$$

respectively. The operator $A$ is called diagonal (resp. off-diagonal) if $A=A^{\mathrm{D}}$ (resp. $A=A^{\mathrm{OD}}$ ).

We collect in the following lemma two simple properties of diagonal and off-diagonal operators in a general Hilbert space $\mathcal{H}$, whose proof is elementary.

Lemma 6.2. Let $A$ be an operator acting in $\mathcal{H}$ and $\Pi$ an orthogonal projection on $\mathcal{H}$ such that $A \Pi$ is densely defined.

1. $A$ is diagonal if and only if $[A, \Pi]=0$. A is off-diagonal if and only if $A=A \Pi+\Pi A$.

2. $A^{\mathrm{OD}}=[[A, \Pi], \Pi]$.

\footnotetext{
${ }^{17}$ The operator $A$ may be unbounded and thus a careful analysis is required. In particular, we want to avoid pathological examples and have $A \supseteq A^{\mathrm{D}}+A^{\mathrm{OD}}$ on a dense domain. Later, we will, for example, apply the block decomposition with respect to the Fermi projection $\Pi_{0}$ to the operator $X_{i}$ for $i \in\{1, \ldots, d\}$ (see Sect. 5.1), and $X_{i} \Pi_{0}$ is densely defined under Assumption 3.1 on the unperturbed Hamiltonian (compare Corollary 3.6).
} 


\subsection{Inverse Liouvillian}

We study here the Liouvillian (super-)operator $B \mapsto\left[H_{0}, B\right]$ associated with the unperturbed Hamiltonian, and in particular the possibility to invert it away from its kernel. We look in other words for the solution $B$ to the equation $\left[H_{0}, B\right]=A$, where $A \in \mathcal{P}\left(\mathcal{H}_{\mathrm{f}}\right)$ is off-diagonal with respect to the decomposition $\mathcal{H}=\Pi_{0} \mathcal{H} \oplus\left(\mathbb{1}-\Pi_{0}\right) \mathcal{H}$. We state in the following proposition the solution to this problem, which traces back at least to $[47,48]$.

Proposition 6.3. Under Assumption 3.1, let $A \in \mathcal{P}\left(\mathcal{H}_{\mathrm{f}}\right)$ be such that $A=A^{\mathrm{OD}}$ with respect to $\Pi_{0}$. Then, the operator $\mathcal{I}(A)$, defined in $(4.2)$, is the unique off-diagonal solution in $\mathcal{P}\left(\mathcal{H}_{\mathrm{f}}, \mathcal{D}_{\mathrm{f}}\right)$ to the equation

$$
\left[H_{0}, \mathcal{I}(A)\right]=A \quad \text { on } \quad \mathcal{U}_{\mathrm{BF}}^{-1} L_{\varrho}^{2}\left(\mathbb{R}^{d}, \mathcal{D}_{\mathrm{f}}\right) .
$$

Proof. From the very definition (4.2) and our hypotheses on $A$, we have that $\mathcal{I}(A)$ is off-diagonal and is in $\mathcal{P}\left(\mathcal{H}_{\mathrm{f}}, \mathcal{D}_{\mathrm{f}}\right)$ by Lemma 3.5 .

Thus, we need only to prove (6.1). Since $H_{0}(k) \in \mathcal{L}\left(\mathcal{D}_{\mathrm{f}}, \mathcal{H}_{\mathrm{f}}\right)$ and $\operatorname{Ran}\left(H_{0}(k)-z \mathbb{1}\right)^{-1} \subset \mathcal{D}_{\mathrm{f}}$ for any $z \in \rho\left(H_{0}\right)$, applying [70, $\S \mathrm{V} .5$ Corollary 2] we have that on $\mathcal{D}_{\mathrm{f}}$

$$
\begin{aligned}
& {\left[H_{0}(k), \mathcal{I}(A)(k)\right]} \\
& \quad=\frac{\mathrm{i}}{2 \pi} \oint_{C} \mathrm{~d} z\left[H_{0}(k),\left(H_{0}(k)-z \mathbb{1}\right)^{-1}\left[A(k), \Pi_{0}(k)\right]\left(H_{0}(k)-z \mathbb{1}\right)^{-1}\right] .
\end{aligned}
$$

Hence, we obtain that on the domain $\mathcal{U}_{\mathrm{BF}}^{-1} L_{\varrho}^{2}\left(\mathbb{R}^{d}, \mathcal{D}_{\mathrm{f}}\right)$

$$
\begin{aligned}
{\left[H_{0}, \mathcal{I}(A)\right] } & =\frac{\mathrm{i}}{2 \pi} \oint_{C} \mathrm{~d} z\left[H_{0}-z \mathbb{1},\left(H_{0}-z \mathbb{1}\right)^{-1}\left[A, \Pi_{0}\right]\left(H_{0}-z \mathbb{1}\right)^{-1}\right] \\
& =\frac{\mathrm{i}}{2 \pi} \oint_{C} \mathrm{~d} z\left[\left[A, \Pi_{0}\right],\left(H_{0}-z \mathbb{1}\right)^{-1}\right]=\left[\left[A, \Pi_{0}\right], \Pi_{0}\right]=A^{\mathrm{OD}}=A,
\end{aligned}
$$

using the Riesz formula (compare (3.5)) and Lemma 6.2.2.

Finally, notice that $\mathcal{I}(A)$ is the unique off-diagonal solution in $\mathcal{P}\left(\mathcal{H}_{\mathrm{f}}, \mathcal{D}_{\mathrm{f}}\right)$ to equation (6.1) for any off-diagonal operator $A \in \mathcal{P}\left(\mathcal{H}_{\mathrm{f}}\right)$. Indeed, if $B \in$ $\mathcal{P}\left(\mathcal{H}_{\mathrm{f}}, \mathcal{D}_{\mathrm{f}}\right)$ is another solution to $(6.1)$, then $\mathcal{I}(A)-B$ commutes with $H_{0}$, and hence with $\Pi_{0}$. By Lemma 6.2.1, $\mathcal{I}(A)-B$ is diagonal, and hence $B=B^{\mathrm{OD}}=$ $\mathcal{I}(A)^{\mathrm{OD}}=\mathcal{I}(A)$.

\subsection{NEASS}

This section is devoted to the proof of Proposition 4.1 and thus to the explicit construction of the NEASS $\Pi^{\varepsilon}$ satisfying $\left(\mathrm{SA}_{1}\right)$ and $\left(\mathrm{SA}_{2}\right)$. In order to give this proof, we first need the following preparatory lemma.

Lemma 6.4. If $A \in \mathcal{P}\left(\mathcal{H}_{\mathrm{f}}, \mathcal{D}_{\mathrm{f}}\right)$, then both $\left(\mathrm{e}^{\varepsilon A}-\mathbb{1}\right)$ and $\overline{\left[\mathrm{e}^{\varepsilon A}, X_{j}\right]}$ are in $\mathcal{P}\left(\mathcal{H}_{\mathrm{f}}, \mathcal{D}_{\mathrm{f}}\right)$ and their norms in this space are bounded uniformly in $\varepsilon \in[0,1]$.

Proof. Clearly, $\left(\mathrm{e}^{\varepsilon A}-\mathbb{1}\right)$ is periodic as $A$ is periodic. Since $\mathcal{L}\left(\mathcal{H}_{\mathrm{f}}, \mathcal{D}_{\mathrm{f}}\right)$ is a Banach space,

$$
\mathrm{e}^{\varepsilon A}(k)-\mathbb{1}=\mathrm{e}^{\varepsilon A(k)}-\mathbb{1}=\sum_{n=1}^{\infty} \frac{\varepsilon^{n} A^{n}(k)}{n !}
$$


converges in $\mathcal{L}\left(\mathcal{H}_{\mathrm{f}}, \mathcal{D}_{\mathrm{f}}\right)$ uniformly in $k \in K$ for any compact set $K \subset \mathbb{R}^{d}$ and $\varepsilon \in[0,1]$, as the sequence $\left\{\sum_{n=1}^{N} \frac{\varepsilon^{n} A^{n}(k)}{n !}\right\}_{N \in \mathbb{N}} \subset \mathcal{L}\left(\mathcal{H}_{\mathrm{f}}, \mathcal{D}_{\mathrm{f}}\right)$ converges absolutely in $\mathcal{L}\left(\mathcal{H}_{\mathrm{f}}, \mathcal{D}_{\mathrm{f}}\right)$ uniformly in $k \in K$ and $\varepsilon \in[0,1]$. Moreover, observe that each summand is such that

$$
\mathbb{R}^{d} \ni k \mapsto \frac{A^{n}(k)}{n !} \in \mathcal{L}\left(\mathcal{H}_{\mathrm{f}}, \mathcal{D}_{\mathrm{f}}\right) \text { is smooth } \forall n \geq 1
$$

and

$$
\sum_{n=1}^{\infty} \frac{\varepsilon^{n} \partial_{k_{j}}\left(A^{n}(k)\right)}{n !}=\sum_{n=1}^{\infty} \frac{\varepsilon^{n}}{n !} \sum_{h=0}^{n-1} A^{h}(k)\left(\partial_{k_{j}} A(k)\right) A^{n-1-h}(k)
$$

converges in $\mathcal{L}\left(\mathcal{H}_{\mathrm{f}}, \mathcal{D}_{\mathrm{f}}\right)$ uniformly in $\varepsilon \in[0,1]$ and in $k \in K$ for any compact set $K \subset \mathbb{R}^{d}$ due to the assumption that $A \in \mathcal{P}\left(\mathcal{H}_{\mathrm{f}}, \mathcal{D}_{\mathrm{f}}\right)$. Therefore, we are allowed to interchange the derivation in $k$ and the series in $n$. Iterating this argument implies that $\left(\mathrm{e}^{\varepsilon A}-\mathbb{1}\right)$ is in $\mathcal{P}\left(\mathcal{H}_{\mathrm{f}}, \mathcal{D}_{\mathrm{f}}\right)$ and that its norm in this space is uniformly bounded with respect to $\varepsilon \in[0,1]$. Thus, by Proposition 3.4 we deduce that $\overline{\left[\mathrm{e}^{\varepsilon A}, X_{j}\right]}=\overline{\left[\mathrm{e}^{\varepsilon A}-\mathbb{1}, X_{j}\right]} \in \mathcal{P}\left(\mathcal{H}_{\mathrm{f}}, \mathcal{D}_{\mathrm{f}}\right)$ again with uniform bounds on its norm for $\varepsilon \in[0,1]$.

We are now ready to tackle the

Proof of Proposition 4.1. In this proof, we will abbreviate the expression "the map $[0,1] \ni \varepsilon \mapsto A^{\varepsilon} \in \mathcal{P}$ is uniformly bounded" for some space of operators $\mathcal{P}$ by just saying that " $A^{\varepsilon}$ is in $\mathcal{P}$ uniformly in $\varepsilon \in[0,1]$."

1, By Corollary 3.6.1 one has that $\left[\overline{\left[X_{j}, \Pi_{0}\right]}, \Pi_{0}\right]$ is in $\mathcal{P}\left(\mathcal{H}_{\mathrm{f}}, \mathcal{D}_{\mathrm{f}}\right) \subset \mathcal{P}\left(\mathcal{H}_{\mathrm{f}}\right)$ and is off-diagonal with respect to $\Pi_{0}$; hence, Proposition 6.3 implies that $\mathcal{S} \in \mathcal{P}\left(\mathcal{H}_{\mathrm{f}}, \mathcal{D}_{\mathrm{f}}\right)$. Self-adjointness of $\mathcal{S}$ is evident.

2 , By Taylor's formula, we find that for any $\varepsilon>0$

$$
\mathrm{e}^{-\mathrm{i} \varepsilon \mathcal{S}} \Pi_{0} \mathrm{e}^{\mathrm{i} \varepsilon \mathcal{S}}=\Pi_{0}+\mathrm{i} \varepsilon\left[\Pi_{0}, \mathcal{S}\right]-\frac{\varepsilon^{2}}{2} \mathrm{e}^{-\mathrm{i} \tilde{\mathcal{E}} \mathcal{S}}\left[\mathcal{S},\left[\mathcal{S}, \Pi_{0}\right]\right] \mathrm{e}^{\mathrm{i} \tilde{\mathcal{E}} \mathcal{S}}
$$

for some $\tilde{\varepsilon} \in(0, \varepsilon)$. Thus, in view of Lemma 6.2 .2 and of the fact that $\overline{\left[X_{j}, \Pi_{0}\right]}=\overline{\left[X_{j}, \Pi_{0}\right]}$ OD , one has

$$
\begin{aligned}
\Pi_{1} & =\mathrm{i}\left[\Pi_{0}, \mathcal{S}\right]=-\left[\Pi_{0}, \mathcal{I}\left(\left[\overline{\left[X_{j}, \Pi_{0}\right]}, \Pi_{0}\right]\right)\right] \\
& =\mathcal{I}\left(\left[\left[\overline{\left[X_{j}, \Pi_{0}\right]}, \Pi_{0}\right], \Pi_{0}\right]\right)=\mathcal{I}\left(\overline{\left[X_{j}, \Pi_{0}\right]}\right) .
\end{aligned}
$$

Moreover,

$$
\Pi_{r}^{\varepsilon}=\frac{\mathrm{i}}{2} \mathrm{e}^{-\mathrm{i} \tilde{\mathcal{S}}}\left[\Pi_{1}, \mathcal{S}\right] \mathrm{e}^{\mathrm{i} \tilde{\varepsilon} \mathcal{S}} .
$$

In view of Corollary 3.6.1 and of Proposition 6.3, we have $\Pi_{1} \in \mathcal{P}\left(\mathcal{H}_{\mathrm{f}}, \mathcal{D}_{\mathrm{f}}\right)$. Notice now that $\left[\Pi_{1}, \mathcal{S}\right]$ is in $\mathcal{P}\left(\mathcal{H}_{\mathrm{f}}, \mathcal{D}_{\mathrm{f}}\right)$ (because $\Pi_{1}, \mathcal{S} \in \mathcal{P}\left(\mathcal{H}_{\mathrm{f}}, \mathcal{D}_{\mathrm{f}}\right)$ ), and $\left(\mathrm{e}^{-\mathrm{i} \tilde{\varepsilon} \mathcal{S}}-\mathbb{1}\right) \in \mathcal{P}\left(\mathcal{H}_{\mathrm{f}}, \mathcal{D}_{\mathrm{f}}\right)$ uniformly in $\tilde{\varepsilon} \in(0, \varepsilon) \subseteq[0,1]$ by Lemma 6.4 . Therefore, we conclude that

$$
\Pi_{r}^{\varepsilon}=\frac{\mathrm{i}}{2}\left(\mathrm{e}^{-\mathrm{i} \tilde{\mathcal{S}}}-\mathbb{1}\right)\left[\Pi_{1}, \mathcal{S}\right] \mathrm{e}^{\mathrm{i} \tilde{\varepsilon} \mathcal{S}}+\frac{\mathrm{i}}{2}\left[\Pi_{1}, \mathcal{S}\right] \mathrm{e}^{\mathrm{i} \tilde{\varepsilon} \mathcal{S}} \in \mathcal{P}\left(\mathcal{H}_{\mathrm{f}}, \mathcal{D}_{\mathrm{f}}\right)
$$

uniformly in $\tilde{\varepsilon} \in(0, \varepsilon) \subseteq[0,1]$. Finally, on the domain $\mathcal{U}_{\mathrm{BF}}^{-1} C_{\varrho}^{\infty}\left(\mathbb{R}^{d}, \mathcal{D}_{\mathrm{f}}\right)$ we have that

$$
\left[H^{\varepsilon}, \Pi^{\varepsilon}\right]=\varepsilon\left(\left[H_{0}, \Pi_{1}\right]-\left[X_{j}, \Pi_{0}\right]\right)+\varepsilon^{2}\left(\left[H^{\varepsilon}, \Pi_{r}^{\varepsilon}\right]-\left[X_{j}, \Pi_{1}\right]\right) .
$$


On the right-hand side, the first term vanishes in view of equation (6.1):

$$
\left[H_{0}, \Pi_{1}\right]=\left[H_{0}, \mathcal{I}\left(\overline{\left[X_{j}, \Pi_{0}\right]}\right)\right]=\left[X_{j}, \Pi_{0}\right] \text { on } \mathcal{U}_{\mathrm{BF}}^{-1} C_{\varrho}^{\infty}\left(\mathbb{R}^{d}, \mathcal{D}_{\mathrm{f}}\right) .
$$

As for the second term, we recognize that

$$
\left[H^{\varepsilon}, \Pi_{r}^{\varepsilon}\right]-\left.\left[X_{j}, \Pi_{1}\right]\right|_{\mathcal{U}_{\mathrm{BF}}^{-1} C_{\varrho}^{\infty}\left(\mathbb{R}^{d}, \mathcal{D}_{\mathrm{f}}\right)}
$$

extends to a bounded operator in $\mathcal{P}\left(\mathcal{H}_{\mathrm{f}}\right)$ uniformly in $\varepsilon \in[0,1]$. Indeed, the second summand $\left.\left[X_{j}, \Pi_{1}\right]\right|_{\mathcal{U}_{\mathrm{BF}}^{-1} C_{\varrho}^{\infty}\left(\mathbb{R}^{d}, \mathcal{D}_{\mathrm{f}}\right)}$ in (6.4) extends to an operator in $\mathcal{P}\left(\mathcal{H}_{\mathrm{f}}, \mathcal{D}_{\mathrm{f}}\right) \subset \mathcal{P}\left(\mathcal{H}_{\mathrm{f}}\right)$ by Proposition 3.4. We split instead the first summand in (6.4) as

$$
\left[H_{0}, \Pi_{r}^{\varepsilon}\right]-\left.\varepsilon\left[X_{j}, \Pi_{r}^{\varepsilon}\right]\right|_{\mathcal{U}_{\mathrm{BF}}^{-1} C_{\varrho}^{\infty}\left(\mathbb{R}^{d}, \mathcal{D}_{\mathrm{f}}\right)} .
$$

The first of the terms above satisfies, in view of (6.3),

$$
\left\|\overline{\left[H_{0}, \Pi_{r}^{\varepsilon}\right]}\right\|_{\mathcal{P}\left(\mathcal{H}_{\mathrm{f}}\right)} \leq 2\left\|H_{0}\right\|_{\mathcal{P}\left(\mathcal{D}_{\mathrm{f}}, \mathcal{H}_{\mathrm{f}}\right)}\left\|\Pi_{r}^{\varepsilon}\right\|_{\mathcal{P}\left(\mathcal{H}_{\mathrm{f}}, \mathcal{D}_{\mathrm{f}}\right)} \text { for } \varepsilon \in[0,1],
$$

while $\left.\left[X_{j}, \Pi_{r}^{\varepsilon}\right]\right|_{\mathcal{U}_{\mathrm{BF}}^{-1} C_{\varrho}^{\infty}\left(\mathbb{R}^{d}, \mathcal{D}_{\mathrm{f}}\right)}$ extends to an operator in $\mathcal{P}\left(\mathcal{H}_{\mathrm{f}}\right)$ applying again Proposition 3.4. By using Leibniz's rule, Lemma 6.4 and Proposition 3.4 one concludes that $\left\|\overline{\left[X_{j}, \Pi_{r}^{\varepsilon}\right]}\right\|_{\mathcal{P}\left(\mathcal{H}_{\mathrm{f}}\right)}$ is bounded uniformly in $\varepsilon \in[0,1]$.

\subsection{Well-Posedness of the Proper $S$-conductivity}

By using (5.2) and combining the following results, here we prove that $\operatorname{Re} \tau\left(J_{\text {prop }, i}^{S} \Pi_{1}\right)=\sigma_{\text {prop }, i j}^{S}$.

Lemma 6.5. Under Assumption 3.1 we have that $\Pi_{0}, \Pi_{1}, \Pi_{r}^{\varepsilon} \in \mathcal{B}_{1}^{\tau}$.

Proof. By Assumption $3.1 \Pi_{0}(k)$ is a finite-rank projection on $\mathcal{H}_{\mathrm{f}}$ with rank $m$ (independent of $k$ ). Thus, in view of Proposition 2.4, we get

$$
\tau\left(\left|\Pi_{0}\right|\right)=\tau\left(\Pi_{0}\right)=\frac{1}{\left|\mathcal{C}_{1}\right|} \operatorname{Tr}\left(\chi_{1} \Pi_{0} \chi_{1}\right)=\frac{1}{\left|\mathcal{C}_{1}\right|} \frac{1}{\left|\mathbb{B}^{d}\right|} \int_{\mathbb{B}^{d}} \mathrm{~d} k \operatorname{Tr}_{\mathcal{H}_{\mathrm{f}}}\left(\Pi_{0}(k)\right)=\frac{m}{\left|\mathcal{C}_{1}\right|}
$$

hence $\Pi_{0} \in \mathcal{B}_{1}^{\tau}$. In view of Propositions 4.1 and 6.3 , we have that $\Pi_{1}=\Pi_{1}^{\mathrm{OD}}$ is off-diagonal with respect to the orthogonal decomposition induced by $\Pi_{0}$, and hence Lemma 6.2.1 implies that $\Pi_{1}=\Pi_{1} \Pi_{0}+\Pi_{0} \Pi_{1}$. Since $\Pi_{1} \in \mathcal{P}\left(\mathcal{H}_{\mathrm{f}}\right) \subset \mathcal{B}_{\infty}^{\tau}$ and $\Pi_{0} \in \mathcal{B}_{1}^{\tau}$ by $(6.5)$, we deduce that the right-hand side of the above is in $\mathcal{B}_{1}^{\tau}$ as $\mathcal{B}_{\infty}^{\tau} \cdot \mathcal{B}_{1}^{\tau} \cdot \mathcal{B}_{\infty}^{\tau} \subset \mathcal{B}_{1}^{\tau}$.

Finally, recall from $(6.2)$ that $\Pi_{r}^{\varepsilon}=\frac{\mathrm{i}}{2} \mathrm{e}^{-\mathrm{i} \tilde{\varepsilon} \mathcal{S}}\left[\Pi_{1}, \mathcal{S}\right] \mathrm{e}^{\mathrm{i} \tilde{\varepsilon} \mathcal{S}}$ for some $\tilde{\varepsilon} \in(0, \varepsilon)$. As we just proved $\Pi_{1} \in \mathcal{B}_{1}^{\tau}$, and the other operators which appear on the righthand side of the above are in $\mathcal{P}\left(\mathcal{H}_{\mathrm{f}}\right) \subset \mathcal{B}_{\infty}^{\tau}$, we conclude that $\Pi_{r}^{\varepsilon} \in \mathcal{B}_{1}^{\tau}$.

Proposition 6.6. Under Assumption 3.1 and the hypotheses on $S$ in Definition 5.1, for $\Pi_{\sharp} \in\left\{\Pi_{0}, \Pi_{1}, \Pi_{r}^{\varepsilon}\right\}$ we have that

1. the operators $\overline{\left[H_{0}, X_{i}\right]} S \Pi_{\sharp}$ and $\left[H_{0}, S\right] \Pi_{\sharp}$ are in $\mathcal{B}_{1}^{\tau}$, 
2. the operators $X_{i}\left[H_{0}, S\right] \Pi_{\sharp}$ and $\overline{\left[H_{0}, X_{i}\right]} S \Pi_{\sharp}$ have finite trace per unit volume.

Proof. 1. We have that $\Pi_{\sharp} \in \mathcal{P}\left(\mathcal{H}_{\mathrm{f}}, \mathcal{D}_{\mathrm{f}}\right)$ by Proposition $4.1, S \in \mathcal{P}\left(\mathcal{D}_{\mathrm{f}}\right)$ by hypothesis, and $\overline{\left[H_{0}, X_{i}\right]} \in \mathcal{P}\left(\mathcal{D}_{\mathrm{f}}, \mathcal{H}_{\mathrm{f}}\right)$ by Corollary 3.6.3; thus, we deduce that

$$
\overline{\left[H_{0}, X_{i}\right]} S \Pi_{\sharp} \in \mathcal{P}\left(\mathcal{D}_{\mathrm{f}}, \mathcal{H}_{\mathrm{f}}\right) \cdot \mathcal{P}\left(\mathcal{D}_{\mathrm{f}}\right) \cdot \mathcal{P}\left(\mathcal{H}_{\mathrm{f}}, \mathcal{D}_{\mathrm{f}}\right) \subset \mathcal{P}\left(\mathcal{H}_{\mathrm{f}}\right) \subset \mathcal{B}_{\infty}^{\tau} .
$$

On the other hand, since

$$
\left[H_{0}, S\right]=H_{0} S-S H_{0} \in \mathcal{P}\left(\mathcal{D}_{\mathrm{f}}, \mathcal{H}_{\mathrm{f}}\right) \cdot \mathcal{P}\left(\mathcal{D}_{\mathrm{f}}\right)+\mathcal{P}\left(\mathcal{H}_{\mathrm{f}}\right) \cdot \mathcal{P}\left(\mathcal{D}_{\mathrm{f}}, \mathcal{H}_{\mathrm{f}}\right) \subset \mathcal{P}\left(\mathcal{D}_{\mathrm{f}}, \mathcal{H}_{\mathrm{f}}\right),
$$

we get that

$$
\left[H_{0}, S\right] \Pi_{\sharp} \in \mathcal{P}\left(\mathcal{D}_{\mathrm{f}}, \mathcal{H}_{\mathrm{f}}\right) \cdot \mathcal{P}\left(\mathcal{H}_{\mathrm{f}}, \mathcal{D}_{\mathrm{f}}\right) \subset \mathcal{P}\left(\mathcal{H}_{\mathrm{f}}\right) \subset \mathcal{B}_{\infty}^{\tau} .
$$

Now we are going to show that the operators in (6.6) and (6.8) are in $\mathcal{B}_{1}^{\tau}$, using the previous results.

First we analyze the case $\Pi_{\sharp}=\Pi_{0}$. In view of Lemma 6.5 we have $\Pi_{0} \in \mathcal{B}_{1}^{\tau}$, thus we deduce that $\overline{\left[H_{0}, X_{i}\right]} S \Pi_{0}=\overline{\left[H_{0}, X_{i}\right]} S \Pi_{0} \cdot \Pi_{0} \in \mathcal{B}_{\infty}^{\tau} \cdot \mathcal{B}_{1}^{\tau} \subset \mathcal{B}_{1}^{\tau}$ and similarly $\left[H_{0}, S\right] \Pi_{0}=\left[H_{0}, S\right] \Pi_{0} \cdot \Pi_{0} \in \mathcal{B}_{\infty}^{\tau} \cdot \mathcal{B}_{1}^{\tau} \subset \mathcal{B}_{1}^{\tau}$.

We proceed with the case $\Pi_{\sharp}=\Pi_{1}$. By virtue of Lemma 6.5 , of the construction in Proposition 4.1, and of Lemma 6.2.1, we have that $\mathcal{B}_{1}^{\tau} \ni \Pi_{1}=$ $\Pi_{1} \Pi_{0}+\Pi_{0} \Pi_{1}$; hence, we obtain that

$$
\overline{\left[H_{0}, X_{i}\right]} S \Pi_{1}=\overline{\left[H_{0}, X_{i}\right]} S \Pi_{1} \cdot \Pi_{0}+\overline{\left[H_{0}, X_{i}\right]} S \Pi_{0} \cdot \Pi_{1} \in \mathcal{B}_{\infty}^{\tau} \cdot \mathcal{B}_{1}^{\tau} \subset \mathcal{B}_{1}^{\tau} .
$$

One can argue in an analogous way to conclude that $\left[H_{0}, S\right] \Pi_{1} \in \mathcal{B}_{1}^{\tau}$ using (6.7).

Finally, we analyze the case $\Pi_{\sharp}=\Pi_{r}^{\varepsilon}=\frac{\mathrm{i}}{2} \mathrm{e}^{-\mathrm{i} \tilde{\varepsilon} \mathcal{S}}\left[\Pi_{1}, \mathcal{S}\right] \mathrm{e}^{\mathrm{i} \tilde{\mathcal{S}}}$. Notice that

$$
\begin{aligned}
\overline{\left[H_{0}, X_{i}\right]} S \Pi_{r}^{\varepsilon}= & \overline{\left[H_{0}, X_{i}\right]} S\left(\mathrm{e}^{-\mathrm{i} \tilde{\varepsilon} \mathcal{S}}-\mathbb{1}\right) \mathrm{e}^{\mathrm{i} \tilde{\mathcal{E}} \mathcal{S}} \Pi_{r}^{\varepsilon}+\frac{\mathrm{i}}{2} \overline{\left[H_{0}, X_{i}\right]} S \Pi_{1} \mathcal{S} \mathrm{e}^{\mathrm{i} \tilde{\varepsilon} \mathcal{S}} \\
& -\frac{\mathrm{i}}{2} \overline{\left[H_{0}, X_{i}\right]} S \mathcal{S} \Pi_{1} \mathrm{e}^{\mathrm{i} \tilde{\varepsilon} \mathcal{S}} .
\end{aligned}
$$

Observe that on the right-hand side of the last equality each summand is in $\mathcal{B}_{1}^{\tau}$. Indeed, since $\left(\mathrm{e}^{-\mathrm{i} \tilde{\mathcal{S}}}-\mathbb{1}\right) \in \mathcal{P}\left(\mathcal{H}_{\mathrm{f}}, \mathcal{D}_{\mathrm{f}}\right)$ by Lemma 6.4 and $\mathrm{e}^{\mathrm{i} \tilde{\varepsilon} \mathcal{S}} \Pi_{r}^{\varepsilon} \in \mathcal{B}_{1}^{\tau}$ by Lemma 6.5, we deduce that $\overline{\left[H_{0}, X_{i}\right]} S\left(\mathrm{e}^{-\mathrm{i} \tilde{\varepsilon} \mathcal{S}}-\mathbb{1}\right) \cdot \mathrm{e}^{\mathrm{i} \tilde{\varepsilon} \mathcal{S}} \Pi_{r}^{\varepsilon} \in \mathcal{P}\left(\mathcal{H}_{\mathrm{f}}\right) \cdot \mathcal{B}_{1}^{\tau} \subset \mathcal{B}_{1}^{\tau}$. Using (6.9) and $\mathcal{S} \mathrm{e}^{\mathrm{i} \tilde{\mathcal{S}} \mathcal{S}} \in \mathcal{P}\left(\mathcal{H}_{\mathrm{f}}\right)$ by Proposition 4.1.1, we infer that $\overline{\left[H_{0}, X_{i}\right]} S \Pi_{1}$. $\mathcal{S} \mathrm{e}^{\mathrm{i} \tilde{\varepsilon} \mathcal{S}} \in \mathcal{B}_{1}^{\tau} \cdot \mathcal{B}_{\infty}^{\tau} \subset \mathcal{B}_{1}^{\tau}$. As for the third summand in (6.10), observe that $\mathcal{S} \in \mathcal{P}\left(\mathcal{H}_{\mathrm{f}}, \mathcal{D}_{\mathrm{f}}\right)$ by Proposition 4.1 .1 and $\Pi_{1} \mathrm{e}^{\mathrm{i} \tilde{\varepsilon} \mathcal{S}} \in \mathcal{B}_{1}^{\tau}$ by Lemma 6.5 , therefore we get that $\overline{\left[H_{0}, X_{i}\right]} S \mathcal{S} \cdot \Pi_{1} \mathrm{e}^{\mathrm{i} \tilde{\varepsilon} \mathcal{S}} \in \mathcal{P}\left(\mathcal{H}_{\mathrm{f}}\right) \cdot \mathcal{B}_{1}^{\tau} \subset \mathcal{B}_{1}^{\tau}$. From a similar argument, it follows that $\left[H_{0}, S\right] \Pi_{r}^{\varepsilon} \in \mathcal{B}_{1}^{\tau}$ owing to $(6.7)$.

2. The conclusion follows by applying part 1 of the statement (which we just proved), Lemma 2.3, Proposition 2.4.1, and Proposition 2.5.1.

In the following lemma, we prove that some expectation values of $J_{\text {conv }, i}^{S}$ which are relevant to transport theory, reduce to the real part of the expectation value of $J_{\text {naive }, i}^{S}:=\mathrm{i} \overline{\left[H_{0}, X_{i}\right]} S$. 
Lemma 6.7. Under Assumption 3.1 and the hypotheses on $S$ in Definition 5.1, let $\Pi_{\sharp} \in\left\{\Pi_{0}, \Pi_{1}, \Pi_{r}^{\varepsilon}, \Pi^{\varepsilon}\right\}$. Then

$$
\tau\left(J_{\text {conv }, i}^{S} \Pi_{\sharp}\right)=\operatorname{Re} \tau\left(\overline{\mathrm{i}\left[H_{0}, X_{i}\right]} S \Pi_{\sharp}\right) .
$$

Proof. We first prove the claim for $\Pi_{\sharp} \in\left\{\Pi_{0}, \Pi^{\varepsilon}\right\}$, exploiting the fact that $\Pi_{\sharp}^{2}=\Pi_{\sharp}=\Pi_{\sharp}^{*}$ in this case. Using in addition that $\Pi_{\sharp} \in \mathcal{B}_{1}^{\tau}$ by Lemma $6.5, J_{\text {naive }, i}^{S} \Pi_{\sharp} \in \mathcal{B}_{\infty}^{\tau}$ by Proposition 6.6 .1 , the cyclicity of the trace per unit volume, $\overline{\operatorname{Tr}(A)}=\operatorname{Tr}\left(A^{*}\right)$, one has that

$$
\begin{aligned}
\operatorname{Re} \tau\left(\overline{\mathrm{i}} \overline{\left[H_{0}, X_{i}\right]} S \Pi_{\sharp}\right) & =\operatorname{Re} \tau\left(\Pi_{\sharp} \mathrm{i} \overline{\left[H_{0}, X_{i}\right]} S \Pi_{\sharp}\right) \\
& =\frac{1}{2}\left(\tau\left(\Pi_{\sharp} J_{\text {naive }, i}^{S} \Pi_{\sharp}\right)+\tau\left(\Pi_{\sharp}\left(J_{\text {naive }, i}^{S}\right)^{*} \Pi_{\sharp}\right)\right) \\
& =\tau\left(\Pi_{\sharp} J_{\text {conv }, i}^{S} \Pi_{\sharp}\right)=\tau\left(J_{\text {conv }, i}^{S} \Pi_{\sharp}\right) .
\end{aligned}
$$

The case $\Pi_{\sharp}=\Pi_{1}$ is subtler, and we crucially use the fact that $\Pi_{1}=$ $\Pi_{1} \Pi_{0}+\Pi_{0} \Pi_{1}$, and $\Pi_{1}^{*}=\Pi_{1}$. Indeed, by using Lemma 2.6, Lemma 6.5, and Proposition 6.6 we obtain that

$$
\begin{aligned}
\operatorname{Re} & \tau\left(J_{\text {naive }, i}^{S} \Pi_{1}\right)=\operatorname{Re} \tau\left(J_{\text {naive }, i}^{S} \Pi_{1} \Pi_{0}\right)+\operatorname{Re} \tau\left(J_{\text {naive }, i}^{S} \Pi_{0} \Pi_{1}\right) \\
= & \frac{1}{2}\left(\tau\left(\Pi_{0} J_{\text {naive }, i}^{S} \Pi_{1}\right)+\tau\left(\Pi_{1} J_{\text {naive }, i}^{S} \Pi_{0}\right)\right) \\
& +\frac{1}{2}\left(\overline{\tau\left(\Pi_{0} J_{\text {naive }, i}^{S} \Pi_{1}\right)}+\overline{\tau\left(\Pi_{1} J_{\text {naive }, i}^{S} \Pi_{0}\right)}\right) \\
= & \frac{1}{2} \tau\left(\overline{\mathrm{i}} \overline{\left[H_{0}, X_{i}\right]} S \Pi_{1}\right)+\frac{1}{2} \tau\left(S \mathrm{i} \overline{\left[H_{0}, X_{i}\right]} \Pi_{1}\right)=\tau\left(J_{\text {conv }, i}^{S} \Pi_{1}\right) .
\end{aligned}
$$

Finally, it remains to prove the claim for $\Pi_{\sharp}=\Pi_{r}^{\varepsilon}$. The latter follows by $\mathbb{R}$-linearity from the previous cases, as $\Pi_{r}^{\varepsilon}=\Pi^{\varepsilon}-\Pi_{0}-\varepsilon \Pi_{1}$.

Proposition 6.8. (Bounds on the remainder terms) Under Assumption 3.1, there exist $C_{1}, C_{2} \in \mathbb{R}$ such that

$$
\left|\tau\left(J_{\text {conv }, i}^{S} \Pi_{r}^{\varepsilon}\right)\right| \leq C_{1} \quad \text { and } \quad\left|\tau\left(J_{\text {prop }, i}^{S} \Pi_{r}^{\varepsilon}\right)\right| \leq C_{2} \quad \forall \varepsilon \in[0,1] .
$$

Proof. We begin by showing the first inequality. In view of Lemma 6.7 and the triangle inequality, we obtain that

$$
\begin{aligned}
\left|\tau\left(J_{\text {conv }, i}^{S} \Pi_{r}^{\varepsilon}\right)\right| \leq & \left|\tau\left(\overline{\mathrm{i}} \overline{\left[H_{0}, X_{i}\right]} S \Pi_{r}^{\varepsilon}\right)\right| \leq\left|\tau\left(\overline{\left[H_{0}, X_{i}\right]} S\left(\mathrm{e}^{-\mathrm{i} \tilde{\mathcal{E}} \mathcal{S}}-\mathbb{1}\right) \mathrm{e}^{\mathrm{i} \tilde{\mathcal{E}} \mathcal{S}} \Pi_{r}^{\varepsilon}\right)\right| \\
& +\frac{1}{2}\left|\tau\left(\overline{\left[H_{0}, X_{i}\right]} S \Pi_{1} \mathcal{S} \mathrm{e}^{\mathrm{i} \tilde{\mathcal{S}}}\right)\right|+\frac{1}{2}\left|\tau\left(\overline{\left[H_{0}, X_{i}\right]} S \mathcal{S} \Pi_{1} \mathrm{e}^{\mathrm{i} \tilde{\mathcal{E}}}\right)\right| .
\end{aligned}
$$

By using the inequalities in (2.10) and Lemma 6.4, the first summand on the right-hand side can be bounded uniformly in $\varepsilon$ as

$$
\left.\left|\tau\left(\overline{\left[H_{0}, X_{i}\right]} S\left(\mathrm{e}^{-\mathrm{i} \tilde{\mathcal{E}} \mathcal{S}}-\mathbb{1}\right) \mathrm{e}^{\mathrm{i} \tilde{\varepsilon} \mathcal{S}} \Pi_{r}^{\varepsilon}\right)\right| \leq C \| \overline{\left[H_{0}, X_{i}\right]}\right]\left\|_{\mathcal{P}\left(\mathcal{D}_{\mathrm{f}}, \mathcal{H}_{\mathrm{f}}\right)}\right\| S\left\|_{\mathcal{P}\left(\mathcal{D}_{\mathrm{f}}\right)}\right\|\left[\Pi_{1}, \mathcal{S}\right] \|_{1, \tau},
$$

where $C$ is a constant independent of $\varepsilon$. Applying again the inequalities in (2.10), we get for the second and third summand in (6.12), respectively

$$
\left|\tau\left(\overline{\left[H_{0}, X_{i}\right]} S \Pi_{1} \mathcal{S} \mathrm{e}^{\mathrm{i} \tilde{\varepsilon} \mathcal{S}}\right)\right| \leq\left\|\overline{\left[H_{0}, X_{i}\right]} S \Pi_{1}\right\|_{1, \tau}\|\mathcal{S}\|
$$


using Proposition 6.6.1 and

$$
\left|\tau\left(\overline{\left[H_{0}, X_{i}\right]} S \mathcal{S} \Pi_{1} \mathrm{e}^{\mathrm{i} \tilde{\mathcal{S}})}\right)\right| \leq\left\|\overline{\left[H_{0}, X_{i}\right]}\right\|_{\mathcal{P}\left(\mathcal{D}_{\mathrm{f}}, \mathcal{H}_{\mathrm{f}}\right)}\|S\|_{\mathcal{P}\left(\mathcal{D}_{\mathrm{f}}\right)}\|\mathcal{S}\|_{\mathcal{P}\left(\mathcal{H}_{\mathrm{f}}, \mathcal{D}_{\mathrm{f}}\right)}\left\|\Pi_{1}\right\|_{1, \tau}
$$

in view of Lemma 6.5 .

Now, to obtain the second inequality of the thesis, notice that

$$
\left|\tau\left(J_{\text {prop }, i}^{S} \Pi_{r}^{\varepsilon}\right)\right| \leq\left|\tau\left(\overline{\left[H_{0}, X_{i}\right]} S \Pi_{r}^{\varepsilon}\right)\right|+\left|\tau\left(X_{i}\left[H_{0}, S\right] \Pi_{r}^{\varepsilon}\right)\right| .
$$

The first summand on the right-hand side is bounded uniformly in $\varepsilon$, as it is shown before, while for the second one we proceed as follows. Since $\left[H_{0}, S\right] \Pi_{r}^{\varepsilon}$ is $\tau$-class by Proposition 6.6.1, Proposition 2.5.1 implies that

$$
\left|\tau\left(X_{i}\left[H_{0}, S\right] \Pi_{r}^{\varepsilon}\right)\right|=\frac{1}{\left|\mathcal{C}_{1}\right|}\left|\operatorname{Tr}\left(\chi_{1} X_{i}\left[H_{0}, S\right] \Pi_{r}^{\varepsilon} \chi_{1}\right)\right| .
$$

Applying the inequality $|\operatorname{Tr}(A B)| \leq\|A\| \operatorname{Tr}(|B|)$ for a bounded operator $A$ and a trace class operator $B$, estimate (2.11) and Proposition 2.4.1, we have that

$$
\begin{aligned}
\frac{1}{\left|\mathcal{C}_{1}\right|}\left|\operatorname{Tr}\left(\chi_{1} X_{i}\left[H_{0}, S\right] \Pi_{r}^{\varepsilon} \chi_{1}\right)\right| & \leq \frac{1}{\left|\mathcal{C}_{1}\right|}\left\|\chi_{1} X_{i} \chi_{1}\right\| \operatorname{Tr}\left(\left|\chi_{1}\left[H_{0}, S\right] \Pi_{r}^{\varepsilon} \chi_{1}\right|\right) \\
& \leq\left\|\chi_{1} X_{i} \chi_{1}\right\|\left\|\left[H_{0}, S\right] \Pi_{r}^{\varepsilon}\right\|_{1, \tau} .
\end{aligned}
$$

Finally, since $\left[H_{0}, S\right] \in \mathcal{P}\left(\mathcal{D}_{\mathrm{f}}, \mathcal{H}_{\mathrm{f}}\right)$ as shown in (6.7), one can reason as in (6.12) to conclude that $\left\|\left[H_{0}, S\right] \Pi_{r}^{\varepsilon}\right\|_{1, \tau} \leq D$, for a constant $D$ independent of $\varepsilon$, which yields the second inequality in the thesis.

\subsection{Chern-Like and Extra Contributions to the $\boldsymbol{S}$-conductivity}

In this subsection, we prove Proposition 5.3, Proposition 5.4, Theorem 5.6 and Lemma 5.10.

Proof of Proposition 5.3. We are going to show that $\mathrm{C}$ and $\mathrm{E}_{\ell}$ are in $\mathcal{B}_{1}^{\tau}$ for any $\ell \in\{1, \ldots, 4\}$ and then equality (5.7) follows from Proposition 2.41. We begin by looking at the Chern-like term $C$. We can write on $\operatorname{Ran}\left(\chi_{L}\right)$ that $\mathrm{C}=\Pi_{0}\left[\overline{\left[X_{i}, \Pi_{0}\right]}, S \overline{\left[X_{j}, \Pi_{0}\right]}\right]$, by using Remark 5.2 and (3.6). In view of Corollary 3.6.1 and Lemma 6.5, we have that $\Pi_{0} \cdot\left[\overline{\left[X_{i}, \Pi_{0}\right]}, S \overline{\left[X_{j}, \Pi_{0}\right]}\right] \in \mathcal{B}_{1}^{\tau} \cdot \mathcal{P}\left(\mathcal{H}_{\mathrm{f}}\right)$. Therefore, the above operators are $\tau$-class as $\mathcal{P}\left(\mathcal{H}_{\mathrm{f}}\right) \cdot \mathcal{B}_{1}^{\tau} \subset \mathcal{B}_{1}^{\tau}$. In view of the cyclicity of the trace per unit volume and the off-diagonality of $\left[X_{i}, \Pi_{0}\right]$, one can rewrite $\tau(\mathrm{C})$ as

$$
\begin{aligned}
\tau\left(\Pi_{0}\left[\left[X_{i}, \Pi_{0}\right], S\left[X_{j}, \Pi_{0}\right]\right]\right) & =\tau\left(\Pi_{0}\left[X_{i}, \Pi_{0}\right] S\left[X_{j}, \Pi_{0}\right]\right)-\tau\left(\Pi_{0} S\left[X_{j}, \Pi_{0}\right]\left[X_{i}, \Pi_{0}\right]\right) \\
& =\tau\left(\Pi_{0}\left[X_{i}, \Pi_{0}\right] S\left[X_{j}, \Pi_{0}\right]\right)-\tau\left(\Pi_{0}\left[X_{j}, \Pi_{0}\right]\left[X_{i}, \Pi_{0}\right] S\right) \\
& =\tau\left(\Pi_{0}\left[\left[X_{i}, \Pi_{0}\right] S,\left[X_{j}, \Pi_{0}\right]\right]\right) .
\end{aligned}
$$

We now analyze the first extra term $E_{1}$. Similarly to the previous computation, we have on $\operatorname{Ran}\left(\chi_{L}\right)$ that $\mathrm{E}_{1}=\mathrm{i} \Pi_{0} \overline{\left[H_{0}, X_{i}\right]} \Pi_{0} S \Pi_{1}+$ $\mathrm{i} \Pi_{0}^{\perp} \overline{\left[H_{0}, X_{i}\right]} \Pi_{0}^{\perp} S \Pi_{1}$. In view of Corollary 3.6.3, Lemma 3.5, Lemma 6.5 and Proposition 6.6.1, we get that $\Pi_{0} \overline{\left[H_{0}, X_{i}\right]} \Pi_{0} S \cdot \Pi_{1} \in \mathcal{P}\left(\mathcal{H}_{\mathrm{f}}\right) \cdot \mathcal{B}_{1}^{\tau}$ and $\Pi_{0}^{\perp} \overline{\left[H_{0}, X_{i}\right]} \Pi_{0}^{\perp} S \Pi_{1}=\Pi_{0}^{\perp} \overline{\left[H_{0}, X_{i}\right]} S \Pi_{1}-\Pi_{0}^{\perp} \overline{\left[H_{0}, X_{i}\right]} \Pi_{0} S \Pi_{1} \in \mathcal{B}_{1}^{\tau}$. Then, noticing that $\Pi_{1}=\Pi_{1}^{\mathrm{OD}}$ by construction (see Propositions 4.1.2 and 6.3) 
and applying Lemma 2.6, we obtain the final expression for $\tau\left(E_{1}\right)$ in (5.9). The proof concerning the statement that $\mathrm{E}_{2}, \mathrm{E}_{3}$ and $\mathrm{E}_{4}$ are in $\mathcal{B}_{1}^{\tau}$ is obtained by similar estimates. By applying Proposition 6.6.2, we infer that $X_{i} \mathrm{R}$ has finite trace per unit volume for any $i \in\{1, \ldots, d\}$ and equality (5.7) is implied by Proposition 2.5.1.

Finally, Lemma 2.6 implies that $\tau\left(\mathrm{E}_{2}\right)=0=\tau\left(\mathrm{E}_{4}\right)$. Indeed, for the first identity observe that $\tau\left(X_{i}^{\mathrm{OD}} S \Pi_{1} H_{0}\right)=\tau\left(\Pi_{1} H_{0} X_{i}^{\mathrm{OD}} S\right)=\tau\left(H_{0} X_{i}^{\mathrm{OD}} S \Pi_{1}\right)$, and for the second one just notices that $\mathrm{E}_{4}$ is the commutator of $\overline{\left[X_{i}, \Pi_{0}\right]} \in \mathcal{B}_{\infty}^{\tau}$ and $\Pi_{0} S \overline{\left[\Pi_{0}, X_{j}\right]} \in \mathcal{B}_{1}^{\tau}$.

Proof of Proposition 5.4. 1. First of all, notice that $\left[H_{0}, B\right] \Pi_{1} \in \mathcal{B}_{1}^{\tau}$ since $\Pi_{1}=$ $\Pi_{1} \Pi_{0}+\Pi_{0} \Pi_{1}$ and, for $\Pi_{\sharp} \in\left\{\Pi_{0}, \Pi_{1}\right\}$, one has $\left[H_{0}, B\right] \cdot \Pi_{\sharp} \in \mathcal{P}\left(\mathcal{D}_{\mathrm{f}}, \mathcal{H}_{\mathrm{f}}\right)$. $\mathcal{P}\left(\mathcal{H}_{\mathrm{f}}, \mathcal{D}_{\mathrm{f}}\right) \subset \mathcal{P}\left(\mathcal{H}_{\mathrm{f}}\right)$ and $\Pi_{\sharp} \in \mathcal{P}\left(\mathcal{H}_{\mathrm{f}}, \mathcal{D}_{\mathrm{f}}\right) \cap \mathcal{B}_{1}^{\tau}$. Since

$$
\Pi_{1}=\frac{\mathrm{i}}{2 \pi} \oint_{C} \mathrm{~d} z\left(H_{0}-z \mathbb{1}\right)^{-1}\left[\overline{\left[X_{j}, \Pi_{0}\right]}, \Pi_{0}\right]\left(H_{0}-z \mathbb{1}\right)^{-1}
$$

by construction (see Propositions 4.1.2 and 6.3), we have that

$$
\begin{aligned}
\mathrm{i} \tau\left(\left[H_{0}, B\right] \Pi_{1}\right) & =-\frac{1}{2 \pi} \oint_{C} \mathrm{~d} z \tau\left(\left(H_{0}-z \mathbb{1}\right)^{-1}\left[H_{0}, B\right]\left(H_{0}-z \mathbb{1}\right)^{-1}\left[\overline{\left[X_{j}, \Pi_{0}\right]}, \Pi_{0}\right]\right) \\
& =-\frac{1}{2 \pi} \oint_{C} \mathrm{~d} z \tau\left(\left[B,\left(H_{0}-z \mathbb{1}\right)^{-1}\right]\left[\overline{\left[X_{j}, \Pi_{0}\right]}, \Pi_{0}\right]\right) \\
& =\mathrm{i} \tau\left(\left[B, \Pi_{0}\right]\left[\overline{\left[X_{j}, \Pi_{0}\right]}, \Pi_{0}\right]\right),
\end{aligned}
$$

where in the first equality we have used the cyclicity of the trace per unit volume, based on the fact that $\left[H_{0}, B\right]\left(H_{0}-z \mathbb{1}\right)^{-1} \in \mathcal{B}_{\infty}^{\tau}$ by Lemma 3.5, and that $\left[\overline{\left[X_{j}, \Pi_{0}\right]}, \Pi_{0}\right] \in \mathcal{B}_{1}^{\tau}$ by Corollary 3.6.1 and Lemma 6.5.

Finally, in virtue of $\left[B, \Pi_{0}\right] \overline{\left[X_{j}, \Pi_{0}\right]} \Pi_{0}=\Pi_{0}\left[B, \Pi_{0}\right] \overline{\left[X_{j}, \Pi_{0}\right]}$ and by using again the cyclicity of the trace per unit volume since $\Pi_{0} \overline{\left[X_{j}, \Pi_{0}\right]} \in \mathcal{B}_{1}^{\tau}$ and $\left[B, \Pi_{0}\right] \in \mathcal{B}_{\infty}^{\tau}$, we conclude that

$$
\begin{aligned}
\tau\left(\mathrm{i}\left[B, \Pi_{0}\right]\left[\overline{\left[X_{j}, \Pi_{0}\right]}, \Pi_{0}\right]\right) & =\tau\left(\mathrm{i} \Pi_{0}\left[B, \Pi_{0}\right] \overline{\left[X_{j}, \Pi_{0}\right]}\right)-\tau\left(\mathrm{i} \Pi_{0} \overline{\left[X_{j}, \Pi_{0}\right]}\left[B, \Pi_{0}\right]\right) \\
& =\tau\left(\mathrm{i} \Pi_{0}\left[\left[B, \Pi_{0}\right], \overline{\left[X_{j}, \Pi_{0}\right]}\right]\right) .
\end{aligned}
$$

2. In view of intermediate formula (6.14), the claim is equivalent to show that $\tau\left(\left[B, \Pi_{0}\right]\left[\overline{\left[X_{j}, \Pi_{0}\right]}, \Pi_{0}\right]\right)=0$. By algebraic manipulations, exploiting the fact that $\Pi_{0}$ is a projection, we obtain that on $\operatorname{Ran}\left(\chi_{1}\right)$

$$
\left[B, \Pi_{0}\right]\left[\overline{\left[X_{j}, \Pi_{0}\right]}, \Pi_{0}\right]=\Pi_{0}^{\perp} B \Pi_{0} \overline{\left[\Pi_{0}, X_{j}\right]}+\Pi_{0} B \Pi_{0}^{\perp} \overline{\left[\Pi_{0}, X_{j}\right]} .
$$

Therefore, since each summand on the right-hand side above is in $\mathcal{B}_{1}^{\tau}$, using again the cyclicity of the trace per unit volume and the off-diagonality of $\overline{\left[\Pi_{0}, X_{j}\right]}$, we get that

$$
\begin{aligned}
& \tau\left(\left[B, \Pi_{0}\right]\left[\overline{\left[X_{j}, \Pi_{0}\right]}, \Pi_{0}\right]\right)=\tau\left(B \Pi_{0} \overline{\left[\Pi_{0}, X_{j}\right]} \Pi_{0}^{\perp}\right)+\tau\left(B \Pi_{0}^{\perp} \overline{\left[\Pi_{0}, X_{j}\right]} \Pi_{0}\right) \\
& =\tau\left(B \overline{\left[\Pi_{0}, X_{j}\right]}\right)=\frac{1}{\left|\mathcal{C}_{1}\right|} \operatorname{Tr}\left(\chi_{1}\left[B \Pi_{0}, X_{j}\right] \chi_{1}\right) \\
& =\frac{1}{\left|\mathcal{C}_{1}\right|}\left\{\operatorname{Tr}\left(\chi_{1} B \Pi_{0} \chi_{1} X_{j} \chi_{1}\right)-\operatorname{Tr}\left(\chi_{1} X_{j} \chi_{1} B \Pi_{0} \chi_{1}\right)\right\}=0,
\end{aligned}
$$


where we have used that $\left[B, X_{j}\right]=0$ and the conditional cyclicity of the trace, since $\chi_{1} B \Pi_{0} \chi_{1}$ is trace class in view of the fact that $B \Pi_{0} \in \mathcal{B}_{1}^{\tau}$ and of Lemma 2.3.

Proof of Theorem 5.6. First of all, Proposition 6.6.2 implies that $J_{\text {prop }, i}^{S} \Pi_{\sharp}$ has a finite trace per unit volume for $\Pi_{\sharp} \in\left\{\Pi_{0}, \Pi_{1}, \Pi_{r}^{\varepsilon}\right\}$, thus all the terms appearing in (5.2) are finite. By virtue of Proposition 6.8, we obtain that $\sigma_{\text {prop }, i j}^{S}=$ $\operatorname{Re} \tau\left(J_{\text {prop }, i}^{S} \Pi_{1}\right)$. Now, notice that $\operatorname{Re} \tau\left(\mathrm{i} \overline{\left[H_{0}, X_{i}\right]} S \Pi_{1}\right)=\tau\left(J_{\text {conv }, i}^{S} \Pi_{1}\right)$ by Lemma 6.7. Therefore, by previous computation in (5.3) and (5.4), we get that

$\operatorname{Re} \tau\left(J_{\text {prop }, i}^{S} \Pi_{1}\right)=\operatorname{Re} \tau\left(\mathrm{i}\left[H_{0}, X_{i}\right] S \Pi_{1}\right)+\operatorname{Re} \tau\left(X_{i} \mathrm{R}\right)=\tau\left(J_{\text {conv }, i}^{S} \Pi_{1}\right)+\operatorname{Re} \tau\left(X_{i} \mathrm{R}\right)$.

Observe that Proposition 6.8 implies that $\sigma_{\text {conv }, i j}^{S}=\operatorname{Re} \tau\left(J_{\text {conv }, i}^{S} \Pi_{1}\right)=$ $\tau\left(J_{\text {conv }, i}^{S} \Pi_{1}\right)$. From equation (5.5) and Proposition 5.3 we derive formula (5.12). Corollary 5.5 along with Proposition 2.5.2 implies the well-posedness of the rotation $S$-conductivity, as it appears in (5.13).

Proof of Lemma 5.10. Obviously, $\mathrm{E}_{3}=0$ and $\mathrm{R}=0$ by using (5.16). Proposition 5.3 implies that $\tau\left(\mathrm{E}_{1}\right)=\mathrm{i} \tau\left(\left[H_{0}, X_{i}^{\mathrm{D}}\right] S^{\mathrm{OD}} \Pi_{1}\right)=0$, since $S^{\mathrm{OD}}=0$.

\section{Acknowledgements}

We are very grateful to Domenico Monaco for intensive discussions and exchange of ideas in an early stage of our project. We are thankful to Luca Fresta, Vojkan Jakšić, Marcello Porta, and Clément Tauber for intensive and fruitful discussions. S. T. also benefited from useful and stimulating interactions with Giuseppe De Nittis and Max Lein. Financial support from the German Science Foundation within the Research Training Group 1838 on "Spectral theory and dynamics of quantum systems" is gratefully acknowledged.

Funding Open access funding provided by UniversitA degli Studi di Roma La Sapienza within the CRUI-CARE Agreement.

Open Access. This article is licensed under a Creative Commons Attribution 4.0 International License, which permits use, sharing, adaptation, distribution and reproduction in any medium or format, as long as you give appropriate credit to the original author(s) and the source, provide a link to the Creative Commons licence, and indicate if changes were made. The images or other third party material in this article are included in the article's Creative Commons licence, unless indicated otherwise in a credit line to the material. If material is not included in the article's Creative Commons licence and your intended use is not permitted by statutory regulation or exceeds the permitted use, you will need to obtain permission directly from the copyright holder. To view a copy of this licence, visit http://creativecommons.org/ licenses/by/4.0/.

Publisher's Note Springer Nature remains neutral with regard to jurisdictional claims in published maps and institutional affiliations. 


\section{Appendix A. Unit Cell Consistency and Vanishing of Persistent $S$-currents}

\section{A.1. Results on the Unit Cell Consistency}

As a preliminary step, we prove that the trace per unit volume, acting on a suitable class of operators, is independent of the choice of the fundamental cell $\mathcal{C}_{1}$. First, notice that chosen any two primitive cells of arbitrary shape, it is possible to cut the first up into pieces, which, when translated through suitable lattice vectors, can be reassembled to give the second. This fact, wellknown to solid state physicists [4], can be reformulated in the following lemma, whose proof is elementary. Recall that $\mathcal{C}_{L}$ is defined in (2.7) with reference to a linear basis $\left\{a_{1}, \ldots, a_{d}\right\}$ of $\Gamma$, while $\widetilde{\mathcal{C}}_{L}$ in (2.17) refers to another linear basis $\left\{\tilde{a}_{1}, \ldots, \tilde{a}_{d}\right\}$ of $\Gamma$.

Lemma A.1. Let $\mathcal{C}_{1}$ and $\widetilde{\mathcal{C}}_{1}$ be two primitive cells. Then there exist a finite set $I \subset \Gamma$ and a family of subsets $\left\{P_{\gamma}\right\}_{\gamma \in I} \subset \mathcal{X}$ such that ${ }^{(7)}$

$$
\mathcal{C}_{1}=\bigsqcup_{\gamma \in I} \mathrm{~T}_{\gamma} P_{\gamma} \quad \text { and } \quad \widetilde{\mathcal{C}}_{1}=\bigsqcup_{\gamma \in I} P_{\gamma} .
$$

In particular, one may choose

$$
P_{\gamma}:=\mathrm{T}_{-\gamma} \mathcal{C}_{1} \cap \widetilde{\mathcal{C}}_{1} \subset \widetilde{\mathcal{C}}_{1} .
$$

Denoting by $\tau(\cdot)$ and $\widetilde{\tau}(\cdot)$, respectively, the trace per unit volume induced by the choice of the primitive cells $\mathcal{C}_{1}$ and $\widetilde{\mathcal{C}}_{1}$, we have the following result.

Proposition A.2. Consider an operator A which is periodic and trace class on compact sets.

1. Then, $\tau(A)=\widetilde{\tau}(A)$.

2. If, in addition, $\operatorname{Tr}\left(\chi_{P_{\gamma}} A \chi_{P_{\gamma}}\right)=0$ for all $\gamma \in I$, where $\left\{P_{\gamma}\right\}_{\gamma \in I}$ are the sets defined in (A.2), then $\tau\left(X_{i} A\right)=\widetilde{\tau}\left(X_{i} A\right)$.

Proof. 1. In view of Proposition 2.4.1, it suffices to prove

$$
\frac{1}{\left|\mathcal{C}_{1}\right|} \operatorname{Tr}\left(\chi_{1} A \chi_{1}\right)=\frac{1}{\left|\widetilde{\mathcal{C}}_{1}\right|} \operatorname{Tr}\left(\widetilde{\chi}_{1} A \tilde{\chi}_{1}\right) \text {. }
$$

Obviously, from decompositions (A.1) and the translation invariance of the Lebesgue measure, it follows that $\left|\mathcal{C}_{1}\right|=\left|\widetilde{\mathcal{C}}_{1}\right|$. The first identity in (A.1), conditional cyclicity of the trace and identity $\chi_{\mathrm{T}_{\gamma} P_{\gamma}}^{2}=\chi_{\mathrm{T}_{\gamma} P_{\gamma}}$ imply that

$$
\operatorname{Tr}\left(\chi_{1} A \chi_{1}\right)=\sum_{\gamma \in I} \operatorname{Tr}\left(\chi_{\mathrm{T}_{\gamma} P_{\gamma}} A \chi_{\mathrm{T}_{\gamma} P_{\gamma}}\right)=\sum_{\gamma \in I} \operatorname{Tr}\left(T_{\gamma} \chi_{P_{\gamma}} T_{\gamma}^{*} A T_{\gamma} \chi_{P_{\gamma}} T_{\gamma}^{*}\right) .
$$

Now, by the invariance of trace under unitary conjugation and the periodicity of $A$, one has that

$$
\operatorname{Tr}\left(\chi_{1} A \chi_{1}\right)=\sum_{\gamma \in I} \operatorname{Tr}\left(\chi_{P_{\gamma}} A \chi_{P_{\gamma}}\right)=\operatorname{Tr}\left(\widetilde{\chi}_{1} A \widetilde{\chi}_{1}\right),
$$


where in the last equality we used the second decomposition in (A.1).

2. After arguing as in the steps leading to (A.3), one notices that

$$
\begin{aligned}
\operatorname{Tr}\left(T_{\gamma} \chi_{P_{\gamma}} T_{\gamma}^{*} X_{i} A T_{\gamma} \chi_{P_{\gamma}} T_{\gamma}^{*}\right) & =\operatorname{Tr}\left(\chi_{P_{\gamma}} T_{\gamma}^{*} X_{i} A T_{\gamma} \chi_{P_{\gamma}}\right)=\operatorname{Tr}\left(\chi_{P_{\gamma}} T_{\gamma}^{*} X_{i} T_{\gamma} A \chi_{P_{\gamma}}\right) \\
& =\gamma_{i} \operatorname{Tr}\left(\chi_{P_{\gamma}} A \chi_{P_{\gamma}}\right)+\operatorname{Tr}\left(\chi_{P_{\gamma}} X_{i} A \chi_{P_{\gamma}}\right)
\end{aligned}
$$

where we have used $\left[T_{\gamma}^{*}, X_{i}\right]=\gamma_{i} T_{\gamma}^{*}$. Therefore, in view of the decompositions (A.1), we conclude that

$$
\operatorname{Tr}\left(\chi_{1} X_{i} A \chi_{1}\right)=\sum_{\gamma \in I} \gamma_{i} \operatorname{Tr}\left(\chi_{P_{\gamma}} A \chi_{P_{\gamma}}\right)+\operatorname{Tr}\left(\tilde{\chi}_{1} X_{i} A \tilde{\chi}_{1}\right)=\operatorname{Tr}\left(\tilde{\chi}_{1} X_{i} A \tilde{\chi}_{1}\right) .
$$

which yields the claim.

For any $\gamma \in I$, consider the operator $\mathbb{1}_{\left\{T_{\eta} T_{\gamma} P_{\gamma}: \eta \in \Gamma\right\}}=\mathbb{1}_{\left\{T_{\eta} P_{\gamma}: \eta \in \Gamma\right\}}$, which is periodic by its very definition (here $\mathbb{1}_{\Omega}$ is an alternative notation for the characteristic function of the set $\Omega$ ). By applying (2.13), one has

$$
\left|\mathcal{C}_{1}\right| \tau\left(\mathbb{1}_{\left\{T_{\eta} P_{\gamma}: \eta \in \Gamma\right\}} A\right)=\operatorname{Tr}\left(\chi_{1} \mathbb{1}_{\left\{T_{\eta} P_{\gamma}: \eta \in \Gamma\right\}} A \chi_{1}\right)=\operatorname{Tr}\left(\chi_{P_{\gamma}} A \chi_{P_{\gamma}}\right),
$$

for every operator $A$ which is periodic and trace class on compact sets. Using the previous rewriting, we are in position to prove that $\chi_{P_{\gamma}} \mathrm{R} \chi_{P_{\gamma}}$, defined in (1.12), has vanishing trace whenever the model enjoys a discrete rotational symmetry.

\section{A.2. Models with Discrete Rotational Symmetries}

Let us fix indices $i \neq j \in\{1, \ldots, d\}$, and denote by $\mathrm{R}_{\vartheta,(i j)}$ the counterclockwise rotation of angle $\vartheta \in[0,2 \pi)$ in the plane $\left(x_{i}, x_{j}\right)$ :

$$
\begin{aligned}
& \mathrm{R}_{\vartheta,(i j)}\left(x_{1}, \ldots, x_{i}, \ldots, x_{j}, \ldots, x_{d}\right) \\
& \quad:=\left(x_{1}, \ldots,(\cos \vartheta) x_{i}-(\sin \vartheta) x_{j}, \ldots,(\sin \vartheta) x_{i}+(\cos \vartheta) x_{j}, \ldots, x_{d}\right) .
\end{aligned}
$$

Rotation operators in the plane $\left(x_{i}, x_{j}\right)$ on $\mathcal{H}$ are defined via

$$
\left(R_{\vartheta,(i j)} \psi\right)(x):=\rho_{\vartheta,(i j)} \psi\left(\mathrm{R}_{\vartheta,(i j)}^{-1} x\right), \quad \text { for } \psi \in \mathcal{H},
$$

where $\rho_{\vartheta,(i j)}$ is a unitary matrix acting on $\mathbb{C}^{N} \cdot{ }^{18}$

Suppose that the $d$-dimensional crystal under consideration is invariant under a rotation of angle $\vartheta=2 \pi / n$, for some $n \in \mathbb{N}^{*}$, in the plane $\left(x_{i}, x_{j}\right)$, namely $\gamma \in \Gamma$ if and only if $\mathrm{R}_{\vartheta,(i j)} \gamma \in \Gamma$ (then it trivially follows that $x \in \mathcal{X}$ if and only if $\left.\mathrm{R}_{\vartheta,(i j)} x \in \mathcal{X}\right)$. A periodic Hamiltonian $H_{0}$ is said to be itself rotationally symmetric or invariant under rotation of angle $\vartheta$ in the plane $\left(x_{i}, x_{j}\right)$ if and only if $R_{\vartheta,(i j)}^{-1} H_{0} R_{\vartheta,(i j)}=H_{0}$. For example, several models on the honeycomb structure, including e.g. the Kane-Mele model (see [33] or [43, Appendix A]), are invariant under the rotation $R_{2 \pi / 3,(12)}$.

Proposition A.3. Let $\vartheta=2 \pi / n$ for some $n \in \mathbb{N}^{*}$. Let the Bravais lattice $\Gamma$ be invariant under the rotation $\mathrm{R}_{\vartheta,(i j)}$, i.e. $\gamma \in \Gamma$ if and only if $\mathrm{R}_{\vartheta,(i j)} \gamma \in \Gamma$, and

$$
R_{\vartheta,(i j)}^{-1} \mathbb{1}_{\left\{T_{\eta} P_{\gamma}: \eta \in \Gamma\right\}} R_{\vartheta,(i j)}=\mathbb{1}_{\left\{T_{\eta} P_{\gamma}: \eta \in \Gamma\right\}} \quad \forall \gamma \in I
$$

\footnotetext{
${ }^{18}$ In two-level systems one defines $\rho_{\vartheta,(12)}:=\mathrm{e}^{-\mathrm{i} \vartheta s_{z}}$ to encode the rotation of angle $\theta$ around the $z$-axis on $\mathbb{C}^{2}$.
} 
Let the operator $H_{0}$, as in Assumption 3.1, be rotationally symmetric of angle $\vartheta$ in the plane $\left(x_{i}, x_{j}\right)$. Let $S=\mathbb{1}_{L^{2}(\mathcal{X})} \otimes s$, as in Definition 5.1 , be such that $\rho_{\vartheta,(i j)}^{-1} s \rho_{\vartheta,(i j)}=s$. Then

1. $\operatorname{Tr}\left(\chi_{P_{\gamma}} \mathrm{R} \chi_{P_{\gamma}}\right)=0 \quad \forall \gamma \in I$, where $\mathrm{R}=\mathrm{i}\left[H_{0}, S\right] \Pi_{1}$ and $\left\{P_{\gamma}\right\}_{\gamma \in I}$ are the sets defined in (A.2).

2. the persistent conventional $S$-current vanishes, namely $\tau\left(J_{\text {conv }, i}^{S} \Pi_{0}\right)=0$.

The vanishing of persistent $S$-currents is not expected to hold true in general, i.e. for systems without a discrete symmetry. However, it has been argued that such persistent currents do not contribute to transport [57].

Proof. 1. In view of Proposition 6.6.1 $\mathrm{R} \in \mathcal{B}_{1}^{\tau}$ and thus applying (A.4) the thesis is equivalent to show that $\tau\left(\mathbb{1}_{\left\{T_{\eta} P_{\gamma}: \eta \in \Gamma\right\}} \mathrm{R}\right)=\mathrm{i} \tau\left(\mathbb{1}_{\left\{T_{\eta} P_{\gamma}: \eta \in \Gamma\right\}}\left[H_{0}, S\right] \Pi_{1}\right)=0$. Using the invariance of the trace under unitary conjugation and the identities $R_{\vartheta,(i j)}^{-1} \chi_{1} R_{\vartheta,(i j)}=\tilde{\chi}_{1}, R_{\vartheta,(i j)}^{-1} H_{0} R_{\vartheta,(i j)}=H_{0}$ and $R_{\vartheta,(i j)}^{-1} S R_{\vartheta,(i j)}=S$ and (A.5), and $\Pi_{1}=\mathcal{I}\left(\overline{\left[X_{j}, \Pi_{0}\right]}\right)$ by Proposition 4.1.2, we obtain that

$$
\begin{aligned}
\left|\mathcal{C}_{1}\right| & \tau\left(\mathbb{1}_{\left\{T_{\eta} P_{\gamma}: \eta \in \Gamma\right\}}\left[H_{0}, S\right] \mathcal{I}\left(\left[X_{j}, \Pi_{0}\right]\right)\right)=\operatorname{Tr}\left(\chi_{1} \mathbb{1}_{\left\{T_{\eta} P_{\gamma}: \eta \in \Gamma\right\}}\left[H_{0}, S\right] \mathcal{I}\left(\left[X_{j}, \Pi_{0}\right]\right) \chi_{1}\right) \\
= & \operatorname{Tr}\left(\widetilde{\chi}_{1} \mathbb{1}_{\left\{T_{\eta} P_{\gamma}: \eta \in \Gamma\right\}}\left[H_{0}, S\right] \mathcal{I}\left(\left[R_{\vartheta,(i j)}^{-1} X_{j} R_{\vartheta,(i j)}, \Pi_{0}\right]\right) \widetilde{\chi}_{1}\right) \\
= & \left|\widetilde{\mathcal{C}_{1}}\right| \widetilde{\tau}\left(\mathbb{1}_{\left\{T_{\eta} P_{\gamma}: \eta \in \Gamma\right\}}\left[H_{0}, S\right] \mathcal{I}\left(\left[R_{\vartheta,(i j)}^{-1} X_{j} R_{\vartheta,(i j)}, \Pi_{0}\right]\right)\right) \\
& =\left|\mathcal{C}_{1}\right| \tau\left(\mathbb{1}_{\left\{T_{\eta} P_{\gamma}: \eta \in \Gamma\right\}}\left[H_{0}, S\right] \mathcal{I}\left(\left[R_{\vartheta,(i j)}^{-1} X_{j} R_{\vartheta,(i j)}, \Pi_{0}\right]\right)\right),
\end{aligned}
$$

where we have used Proposition 2.4.1 and Proposition A.2.1. Therefore, by iterating the previous computation we have that

$$
\begin{aligned}
\tau & \left(\mathbb{1}_{\left\{T_{\eta} P_{\gamma}: \eta \in \Gamma\right\}}\left[H_{0}, S\right] \mathcal{I}\left(\left[X_{j}, \Pi_{0}\right]\right)\right) \\
& =\frac{1}{n} \sum_{k=0}^{n-1} \tau\left(\mathbb{1}_{\left\{T_{\eta} P_{\gamma}: \eta \in \Gamma\right\}}\left[H_{0}, S\right] \mathcal{I}\left(\left[R_{\vartheta,(i j)}^{-k} X_{j} R_{\vartheta,(i j)}^{k}, \Pi_{0}\right]\right)\right) .
\end{aligned}
$$

Now we are going to compute $\sum_{k=0}^{n-1} R_{\vartheta,(i j)}^{-k} X_{j} R_{\vartheta,(i j)}^{k}$. The rotation of angle $\vartheta$ acts non-trivially only in the plane $\left(x_{i}, x_{j}\right)$, which we parametrize with the complex coordinate $z:=x_{i}+\mathrm{i} x_{j}$. In this parametrization, the rotation of angle $\vartheta$ is implemented as $\hat{\mathrm{R}}_{\vartheta} z:=\mathrm{e}^{\mathrm{i} \vartheta} z$. Introducing the complex position operator $Z:=X_{i}+\mathrm{i} X_{j}$, one has then that $\hat{R}_{\vartheta,(i j)}^{-k} \mathrm{i} X_{j} \hat{R}_{\vartheta,(i j)}^{k}=\operatorname{Im}\left(\mathrm{e}^{\mathrm{i} k \vartheta} Z\right)$ and thus

$$
\sum_{k=0}^{n-1} \hat{R}_{\vartheta,(i j)}^{-k} \mathrm{i} X_{j} \hat{R}_{\vartheta,(i j)}^{k}=\operatorname{Im}\left(\sum_{k=0}^{n-1} \mathrm{e}^{\mathrm{i} 2 \pi k / n} Z\right) .
$$

As $\sum_{k=0}^{n-1} \mathrm{e}^{\mathrm{i} 2 \pi k / n}=0$, we deduce that the term in (A.6) vanishes. This concludes the proof.

2. In view of decomposition (A.1), it suffices to show that

$$
\operatorname{Tr}\left(\chi_{P_{\gamma}} i\left[H_{0}, X_{i}\right] S \Pi_{0} \chi_{P_{\gamma}}\right)=0 \quad \forall \gamma \in I,
$$

whose proof is analogous to the previous one since $\left[H_{0}, X_{i}\right] S \Pi_{0} \in \mathcal{B}_{1}^{\tau}$. 
Remark A.4. In general, even exploiting the peculiar discrete rotational symmetries in the hypotheses of Proposition A.3, it is not obvious that the persistent proper $S$-current vanishes, i.e. $\tau\left(J_{\text {prop }, i}^{S} \Pi_{0}\right)=0$, since the argument we used relies on the periodicity of the operators involved and $J_{\mathrm{prop}, i}^{S}$ is not periodic. Nevertheless, in the Kane-Mele model this property holds true thanks to the specific structure of the model.

\section{A.3. Vanishing of Persistent $S$-current When $S$ is Conserved}

When $S$ is a conserved quantity, namely when $\left[H_{0}, S\right]=0$, the vanishing of the persistent $S$-current $J_{i}^{S}=J_{\text {prop }, i}^{S}=J_{\text {conv }, i}^{S}$ holds true without any symmetry assumption on $H_{0}$ (compare [10,12], where similar results are deduced in the case $S=\mathbb{1}$, and [8], which offers a proof in the context of many-body quantum spin systems).

To show this, notice first that $\left[\Pi_{0}, S\right]=0$ as well and that $J_{i}^{S}=$ $\mathrm{i} \overline{\left[H_{0}, X_{i}\right]} S$ is a periodic operator in view of Lemma 2.1. Moreover, $J_{i}^{S} \Pi_{0}=$ $\mathrm{i}\left[H_{0}, X_{i}\right] S \Pi_{0}$ is $\tau$-class in view of Proposition 6.6.1. Consequently, by the identity $\Pi_{0}^{2}=\Pi_{0}$, Lemma 2.6 and Proposition 2.4.1, we have that

$$
\tau\left(\overline{\left[H_{0}, X_{i}\right]} S \Pi_{0}\right)=\tau\left(\Pi_{0} \overline{\left[H_{0}, X_{i}\right]} S \Pi_{0}\right)=\frac{1}{\left|\mathcal{C}_{1}\right|} \operatorname{Tr}\left(\chi_{1} \Pi_{0} \overline{\left[H_{0}, X_{i}\right]} S \Pi_{0} \chi_{1}\right) .
$$

By Remark 5.2, after some algebra, we get that

$$
\begin{aligned}
\chi_{1} \Pi_{0} \overline{\left[H_{0}, X_{i}\right]} S \Pi_{0} \chi_{1} & =\chi_{1} \Pi_{0}\left[H_{0}, X_{i}\right] S \Pi_{0} \chi_{1}=\chi_{1}\left[\Pi_{0} H_{0} S \Pi_{0}, \Pi_{0} X_{i} \Pi_{0}\right] \chi_{1} \\
& =\chi_{1}\left[\Pi_{0} H_{0} S \Pi_{0}, X_{i}\right] \chi_{1}-\chi_{1}\left[\Pi_{0} H_{0} S \Pi_{0}, X_{i}^{\mathrm{OD}}\right] \chi_{1} .
\end{aligned}
$$

Notice that the trace of the first summand above vanishes:

$$
\operatorname{Tr}\left(\chi_{1}\left[\Pi_{0} H_{0} S \Pi_{0}, X_{i}\right] \chi_{1}\right)=\operatorname{Tr}\left(\chi_{1} \Pi_{0} H_{0} S \Pi_{0} \chi_{1} X_{i} \chi_{1}-\chi_{1} X_{i} \chi_{1} \Pi_{0} H_{0} S \Pi_{0} \chi_{1}\right),
$$

where both summands inside the trace are trace class. Indeed, $\Pi_{0} \chi_{1}$ is an Hilbert-Schmidt operator, since $\operatorname{Tr}\left(\left(\Pi_{0} \chi_{1}\right)^{*} \Pi_{0} \chi_{1}\right)=\operatorname{Tr}\left(\chi_{1} \Pi_{0} \chi_{1}\right)=$ $\left|\mathcal{C}_{1}\right| \tau\left(\Pi_{0}\right)<\infty$ by Lemma 6.5 . This implies that the adjoint $\chi_{1} \Pi_{0}$ is HilbertSchmidt as well. Since $S, \Pi_{0} H_{0} \Pi_{0}$ and $\chi_{1} X_{i} \chi_{1}$ are all bounded operators, the desired claim follows in view of the conditional cyclicity of the trace $\operatorname{Tr}(\cdot)$. Finally, we have that the trace of the second summand in (A.7) vanishes as well. Indeed, by Remark 5.2, Lemma 6.2.2 and definition (3.6) we obtain that

$$
\chi_{1}\left[\Pi_{0} H_{0} S \Pi_{0}, X_{i}^{\mathrm{OD}}\right] \chi_{1}=\chi_{1}\left[\Pi_{0} H_{0} S \Pi_{0},\left[\overline{\left[X_{i}, \Pi_{0}\right]}, \Pi_{0}\right]\right] \chi_{1}
$$

and thus, using Proposition 2.41, we deduce

$$
\begin{aligned}
\frac{1}{\left|\mathcal{C}_{1}\right|} \operatorname{Tr}\left(\chi_{1}\left[\Pi_{0} H_{0} S \Pi_{0}, X_{i}{ }^{\mathrm{OD}}\right] \chi_{1}\right)= & \tau\left(\Pi_{0} H_{0} S \Pi_{0}\left[\overline{\left[X_{i}, \Pi_{0}\right]}, \Pi_{0}\right]\right)+ \\
& -\tau\left(\left[\overline{\left[X_{i}, \Pi_{0}\right]}, \Pi_{0}\right] \Pi_{0} H_{0} S \Pi_{0}\right) .
\end{aligned}
$$


The conditional cyclicity of $\tau$ implies the conclusion, since $\Pi_{0} H_{0} S \Pi_{0} \in \mathcal{B}_{\infty}^{\tau}$ and $\left[\overline{\left[X_{i}, \Pi_{0}\right]}, \Pi_{0}\right] \in \mathcal{B}_{1}^{\tau}$.

\section{References}

[1] Aizenman, M., Graf, G.M.: Localization bounds for an electron gas. J. Phys. A 31, 6783-6806 (1998)

[2] Aizenman, M., Warzel, S.: Random Operators. Vol. 168 in Graduate Studies in Mathematics. American Mathematical Society, Providence (2015)

[3] Antinucci, G., Mastropietro, V., Porta, M.: Universal edge transport in interacting Hall systems. Commun. Math. Phys. 362, 295-362 (2018)

[4] Ashcroft, N.W., Mermin, N.D.: Solid State Physics, College edn. Harcourt College Publishers, New York (1976)

[5] Avron, J., Seiler, R., Simon, B.: Charge deficiency, charge transport and comparison of dimensions. Commun. Math. Phys. 159, 399-422 (1994)

[6] Bachmann, S., Bols, A., De Roeck, W., Fraas, M.: Quantization of conductance in gapped interacting systems. Ann. H. Poincaré 19, 695-708 (2018)

[7] Bachmann, S., De Roeck, W., Fraas, M.: The adiabatic theorem and linear response theory for extended quantum systems. Commun. Math. Phys. 361, 997-1027 (2018)

[8] Bachmann, S., Bols, A., De Roeck, W., Fraas, M.: A many-body index for quantum charge transport. Commun. Math. Phys. Online First (2019)

[9] Bellissard, J.: $K$-theory of $C^{*}$-algebras in solid state physics. In: Dorlas, T.C., Hugenholtz, N.M., Winnink, M. (eds.) Statistical Mechanics and Field Theory: Mathematical Aspects. Vol. 257 in Lecture Notes in Physics, pp. 99-156. Springer, Berlin (1986)

[10] Bellissard, J., van Elst, A., Schulz-Baldes, H.: The non-commutative geometry of the quantum Hall effect. J. Math. Phys. 35, 5373-5471 (1994)

[11] Birman, S.H., Suslina, T.A.: Periodic magnetic Hamiltonian with a variable metric. The problem of absolute continuity. Algebra i Analiz 11, 1-40 (1999). English translation in St. Petersburg Math. J. 11, 1-30 (2000)

[12] Bouclet, J.-M., Germinet, F., Klein, A., Schenker, J.H.: Linear response theory for magnetic Schrödinger operators in disordered media. J. Funct. Anal. 226, 301-372 (2005)

[13] Bru, J.-B., de Siqueira Pedra, W.: Lieb-Robinson Bounds for Multicommutators and Applications to Response Theory. Vol. 13 in Springer Briefs in Mathematical Physics. Springer, Berlin (2016)

[14] Cánces, E., Fermanian Kammerer, C., Levitt, A., Siraj-Dine, S.: Coherent electronic transport in periodic systems. Preprint arXiv:2002.01990 (2020)

[15] Cornean, H.D., Monaco, D., Teufel, S.: Wannier functions and $\mathbb{Z}_{2}$ invariants in time-reversal symmetric topological insulators. Rev. Math. Phys. 29, 1730001 (2017)

[16] Culcer, D., Yao, Y., Niu, Q.: Coherent wave-packet evolution in coupled bands. Phys. Rev. B 72, 085110 (2005) 
[17] De Nittis, G., Lein, M.: Linear Response Theory: A Modern Analytic-Algebraic Approach. Vol. 21 in Springer Briefs in Mathematical Physics. Springer, Berlin (2017)

[18] Du, L., Knez, I., Sullivan, G., Dui, R.R.: Robust helical edge transport in gated InAs/GaSb bilayers. Phys. Rev. Lett. 114, 096802 (2015)

[19] Elgart, A., Schlein, B.: Adiabatic charge transport and the Kubo formula for Landau-type Hamiltonians. Commun. Pure Appl. Math. 57, 590-615 (2004)

[20] Fiorenza, D., Monaco, D., Panati, G.: $\mathbb{Z}_{2}$-invariants of topological insulators as geometric obstructions. Commun. Math. Phys. 343, 1115-1157 (2016)

[21] Fu, L., Kane, C.L.: Time reversal polarization and a $\mathbb{Z}_{2}$ adiabatic spin pump. Phys. Rev. B 74, 195312 (2006)

[22] Graf, G.M.: Aspects of the integer quantum hall effect. In: Gesztesy, F., Deift, P., Galvez, C., Perry, P., Schlag, W. (eds.) Spectral Theory and Mathematical Physics: A Festschrift in Honor of Barry Simon's 60th Birthday. No. 76 in Proceedings of Symposia in Pure Mathematics, pp. 429-442. American Mathematical Society, Providence (2007)

[23] Graf, G.M., Porta, M.: Bulk-edge correspondence for two-dimensional topological insulators. Commun. Math. Phys. 324, 851-895 (2013)

[24] Giuliani, A., Mastropietro, V., Porta, M.: Universality of the Hall conductivity in interacting electron systems. Commun. Math. Phys. 349, 1107-1161 (2017)

[25] Haldane, F.D.M.: Model for a quantum Hall effect without Landau levels: condensed-matter realization of the "parity anomaly". Phys. Rev. Lett. 61, 2015 (1988)

[26] Hasan, M.Z., Kane, C.L.: Colloquium: topological insulators. Rev. Mod. Phys. 82, 3045-3067 (2010)

[27] Hastings, M., Michalakis, S.: Quantization of Hall conductance for interacting electrons on a torus. Commun. Math. Phys. 334, 433-471 (2015)

[28] Henheik, J., Teufel, S.: Justifying Kubo's formula for gapped systems at zero temperature: a brief review and some new results. Rev. Math. Phys. Online Ready (2020)

[29] Jakšić, V., Ogata, Y., Pillet, C.A.: The Green-Kubo formula and Onsager reciprocity relations in quantum statistical mechanics. Commun. Math. Phys. 265, $721-738$ (2006)

[30] Jakšić, V., Ogata, Y., Pillet, C.A.: Linear response theory for thermally driven quantum open systems. J. Stat. Phys. 123, 547-569 (2006)

[31] Jakšić, V., Ogata, Y., Pillet, C.A.: The Green-Kubo formula for the spin-fermion system. Commun. Math. Phys. 268, 369-401 (2006)

[32] Jakšić, V., Ogata, Y., Pillet, C.A.: The Green-Kubo formula for locally interacting fermionic systems. Ann. H. Poincaré 8, 1013-1036 (2007)

[33] Kane, C.L., Mele, E.J.: Quantum spin Hall effect in graphene. Phys. Rev. Lett. 95, $226801(2005)$

[34] Kane, C.L., Mele, E.J.: $\mathbb{Z}_{2}$ topological order and the quantum spin Hall effect. Phys. Rev. Lett. 95, 146802 (2005)

[35] Kato, T.: Perturbation Theory for Linear Operators. Springer, Berlin (1966)

[36] Katsura, H., Koma, T.: The $\mathbb{Z}_{2}$ index of disordered topological insulators with time-reversal symmetry. J. Math. Phys. 57, 021903 (2016) 
[37] Klein, M., Seiler, R.: Power-law corrections to the Kubo formula vanish in quantum Hall systems. Commun. Math. Phys. 128, 141-160 (1990)

[38] König, M., et al.: The quantum spin Hall effect: theory and experiment. J. Phys. Soc. Jpn. 77, 031007 (2008)

[39] Kubo, R.: Statistical-mechanical theory of irreversible processes I: general theory and simple applications to magnetic and conduction problems. J. Phys. Soc. Jpn. 12, 570-586 (1957)

[40] Kuchment, P.: An overview of periodic ellipic operators. Bull. AMS 53, 343-414 (2016)

[41] Marcelli, G., Teufel, S.: Non-equilibrium almost-stationary states and linear response for gapped non-interacting quantum systems. In preparation (2020)

[42] Marcelli, G.: Improved energy estimates for a class of time-dependent perturbed Hamiltonians. Preprint https://arxiv.org/abs/1904.11300 (2019)

[43] Marcelli, G., Panati, G., Tauber, C.: Spin conductance and spin conductivity in topological insulators: analysis of Kubo-like terms. Ann. H. Poincaré 20, 20712099 (2019)

[44] Mastropietro, V., Porta, M.: Spin Hall insulators beyond the helical Luttinger model. Phys. Rev. B 96, 245135 (2017)

[45] Monaco, D., Panati, G., Pisante, A., Teufel, S.: Optimal decay of Wannier functions in Chern and quantum Hall insulators. Commun. Math. Phys. 359, 61-100 (2018)

[46] Monaco, D., Teufel, S.: Adiabatic currents for interacting electrons on a lattice. Rev. Math. Phys. 31, 1950009 (2019)

[47] Nenciu, G.: Linear adiabatic theory, exponential estimates. Commun. Math. Phys. 152, 479-496 (1993)

[48] Nenciu, G.: On asymptotic perturbation theory for quantum mechanics: almost invariant subspaces and gauge invariant magnetic perturbation theory. J. Math. Phys. 43, 1273 (2002)

[49] Moretti, V., Oppio, M.: The correct formulation of Gleason's theorem in quaternionic Hilbert spaces. Ann. H. Poincaré 19, 3321-3355 (2018)

[50] Panati, G., Pisante, A.: Bloch bundles, Marzari-Vanderbilt functional and maximally localized Wannier functions. Commun. Math. Phys. 322, 835-875 (2013)

[51] Panati, G., Sparber, C., Teufel, S.: Geometric currents in piezoelectricity. Arch. Rat. Mech. Anal. 191, 387-422 (2009)

[52] Panati, G., Spohn, H., Teufel, S.: Effective dynamics for Bloch electrons: Peierls substitution and beyond. Commun. Math. Phys. 242, 547-578 (2003)

[53] Panati, G., Spohn, H., Teufel, S.: Space-adiabatic perturbation theory. Adv. Theor. Math. Phys. 7, 145-204 (2003)

[54] Pastur, L., Figotin, A.: Spectra of Random and Almost-Periodic Operators. Springer, Berlin (1992)

[55] Prodan, E.: An edge index for the quantum spin Hall effect. J. Phys. A: Math. Theor. 42, 1 (2009)

[56] Prodan, E.: Robustness of the spin-Chern number. Phys. Rev. B 80, 125327 (2009)

[57] Rashba, E.I.: Spin currents in thermodynamic equilibrium: the challenge of discerning transport currents. Phys. Rev. B 68, 241315 (2003) 
[58] Schulz-Baldes, H.: Persistence of spin edge currents in disordered quantum spin Hall systems. Commun. Math. Phys. 324, 589-600 (2013)

[59] Schulz-Baldes, H.: $\mathbb{Z}_{2}$-indices and factorization properties of odd symmetric Fredholm operators. Documenta Math. 20, 1481-1500 (2015)

[60] Sheng, L., Sheng, D.N., Ting, C.S., Haldane, F.D.M.: Nondissipative spin Hall effect via quantized edge transport. Phys. Rev. Lett. 95, 136602 (2005)

[61] Sheng, D.N., Weng, Z.Y., Sheng, L., Haldane, F.D.M.: Quantum spin-Hall effect and topologically invariant Chern numbers. Phys. Rev. Lett. 97, 036808 (2006)

[62] Shi, J., Zhang, P., Xiao, D., Niu, Q.: Proper definition of spin current in spinorbit coupled systems. Phys. Rev. Lett. 96, 076604 (2006)

[63] Shindou, R., Imura, K.-I.: Noncommutative geometry and non-abelian Berry phase in the wave-packet dynamics of Bloch electrons. Nucl. Phys. B 720, 399435 (2005)

[64] Sinova, J., Culcer, D., Niu, Q., Sinitsyn, N.A., Jungwirth, T., MacDonald, A.H.: Universal intrinsic spin Hall effect. Phys. Rev. Lett. 92, 126603 (2004)

[65] Sinova, J., Valenzuela, S.O., Wunderlich, J., Back, C.H., Jungwirth, T.: Spin Hall effects. Rev. Mod. Phys. 87, 1213 (2015)

[66] Stiepan, H.-M., Teufel, S.: Semiclassical approximations for hamiltonians with operator-valued symbols. Commun. Math. Phys. 320, 821-849 (2013)

[67] Teufel, S.: Adiabatic Perturbation Theory in Quantum Dynamics. No. 1821 in Lecture Notes in Mathematics. Springer, Berlin (2003)

[68] Teufel, S.: Non-equilibrium almost-stationary states for interacting electrons on a lattice. Commun. Math. Phys. 373, 621-653 (2020)

[69] Yang, X., He, Z., Zheng, X.: Unit cell consistency of maximally localized Wannier functions. Electron. Struct. 2, 1 (2020)

[70] Yosida, K.: Functional Analysis. Springer, Berlin (1980)

[71] Zak, J.: Magnetic translation group. Phys. Rev. 134, A1602 (1964)

[72] Zhang, P., Wang, Z., Shi, J., Xiao, D., Niu, Q.: Theory of conserved spin current and its application to a two-dimensional hole gas. Phys. Rev. B 77, 075304 (2008)

Giovanna Marcelli and Stefan Teufel

Fachbereich Mathematik

Eberhard Karls Universität Tübingen

Auf der Morgenstelle 10

72076 Tübingen

Germany

e-mail: giovanna.marcelli@uni-tuebingen.de;

giovanna.marcelli@sissa.it;

stefan.teufel@uni-tuebingen.de

Gianluca Panati

Dipartimento di Matematica

"La Sapienza" Università di Roma

Piazzale Aldo Moro 2

00185 Rome

Italy

e-mail: panati@mat.uniroma1.it 
Communicated by Vieri Mastropietro.

Received: April 26, 2020.

Accepted: October 10, 2020. 